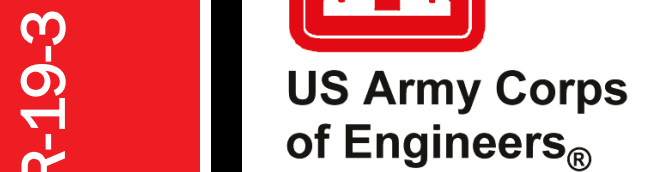

\title{
of Engineers $s_{\circledast}$
}

Engineer Research and

Development Center

\section{Localization and Mapping of Unknown} Locations with Unmanned Ground Vehicles

Doris M. Turnage

February 2019 
The U.S. Army Engineer Research and Development Center (ERDC) solves the nation's toughest engineering and environmental challenges. ERDC develops innovative solutions in civil and military engineering, geospatial sciences, water resources, and environmental sciences for the Army, the Department of Defense, civilian agencies, and our nation's public good. Find out more at www.erdc.usace.army.mil.

To search for other technical reports published by ERDC, visit the ERDC online library at http://acwc.sdp.sirsi.net/client/default. 
ERDC/GSL TR-19-3

February 2019

\section{Localization and Mapping of Unknown Locations with Unmanned Ground Vehicles}

Doris M. Turnage

Geotechnical and Structure Laboratory

U.S. Army Engineer Research and Development Center

3909 Halls Ferry Road

Vicksburg, MS 39180-6199

Final report

Approved for public release; distribution is unlimited.

Prepared for U.S. Army Engineer Research and Development Center Vicksburg, MS 39180-6199 


\section{Abstract}

The main goals of this research are to enhance a commercial off the shelf (COTS) software platform to support unmanned ground vehicles (UGVs) exploring the complex environment of tunnels, to test the platform within a simulation environment, and to validate the architecture through fieldtesting.

Developing this platform enhances the U.S. Army Engineering Research and Development Center's (ERDC's) current capabilities and creates a safe and efficient autonomous vehicle to perform the following functions within tunnels: (1) localizing (e.g., position tracking) and mapping of its environment, (2) traversing varied terrains, (3) sensing the environment for objects of interest, and (4) increasing the level of autonomy of UGVs available at the ERDC.

The simulation experiments were performed in the STAGE Simulator, a physics-based, multi-scale numerical test bed developed by Robotic Operating System (ROS). Physical testing was conducted in Vicksburg, MS, using a Coroware Explorer. Both the simulation and physical testing evaluated three simultaneous localization and mapping (SLAM) algorithms, i.e., Hector SLAM, Gmapping, and CORESLAM to determine the superior algorithm. The superior algorithm was then used to localize the robot to the environment and autonomously travel from a start location to a destination location.

Completion of this research has increased the ERDC's level of autonomy for UGVs from tether to tele-operated to autonomous.

DISCLAIMER: The contents of this report are not to be used for advertising, publication, or promotional purposes. Citation of trade names does not constitute an official endorsement or approval of the use of such commercial products. All product names and trademarks cited are the property of their respective owners. The findings of this report are not to be construed as an official Department of the Army position unless so designated by other authorized documents. 


\section{Contents}

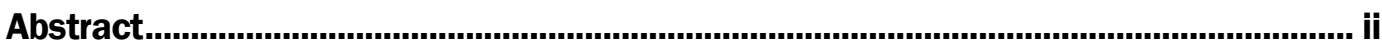

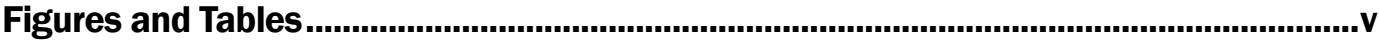

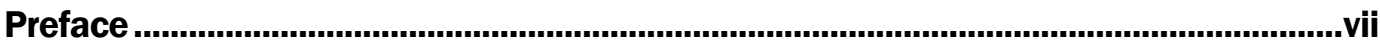

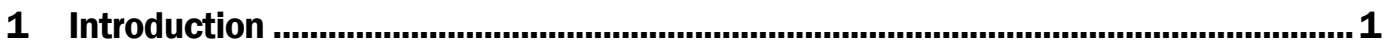

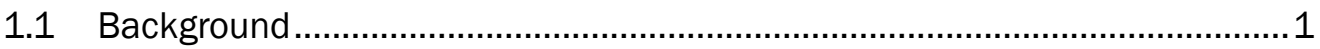

1.2 Types of unmanned vehicles....................................................................

1.2.1 Unmanned ground vehicles.............................................................................. 3

1.2.2 Unmanned aerial vehicles ............................................................................... 4

1.2.3 Autonomous underwater vehicles....................................................................... 4

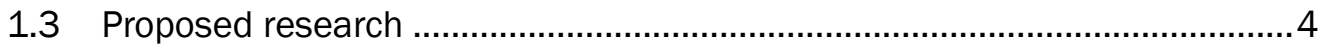

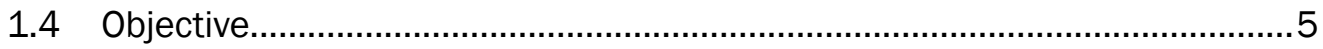

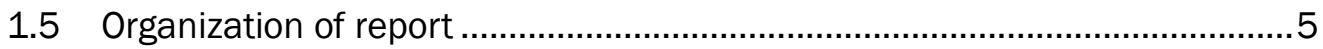

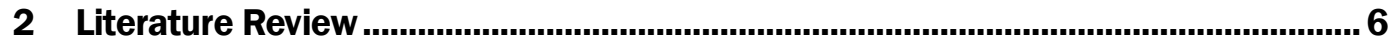

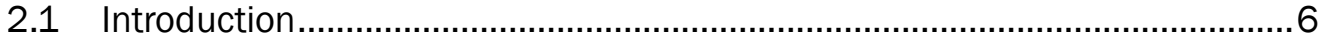

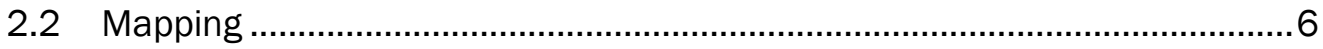

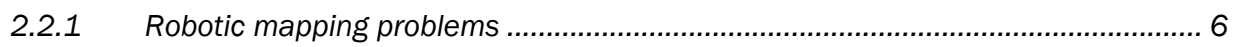

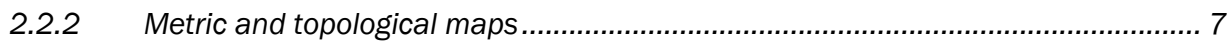

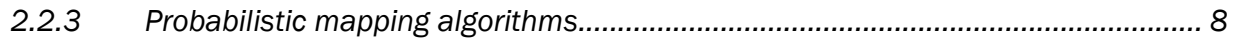

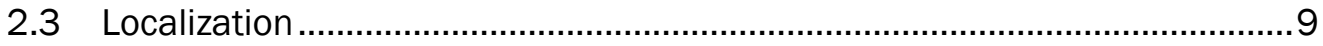

2.3.1 Monte Carlo Localization ............................................................................ 10

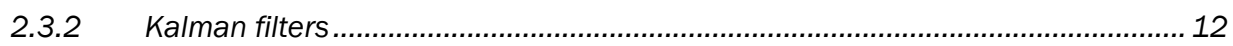

2.3.3 Markov Localization ........................................................................................ 12

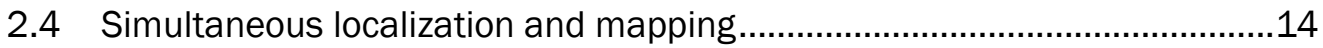

2.5 Research goal ................................................................................. 14

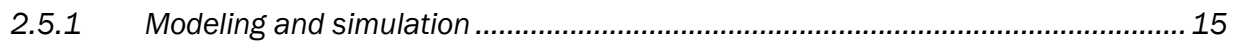

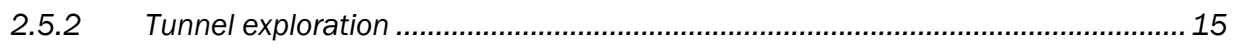

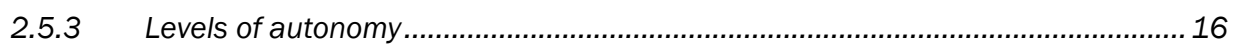

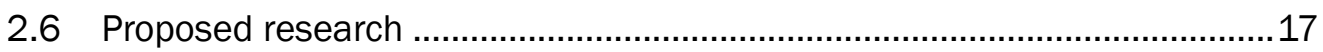

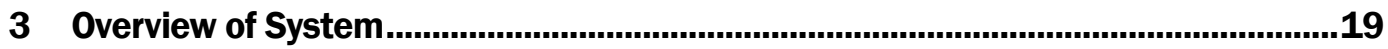

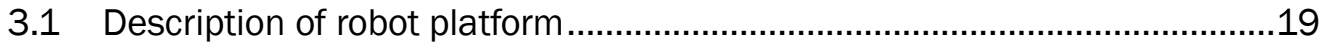

3.2 Description of software ....................................................................21

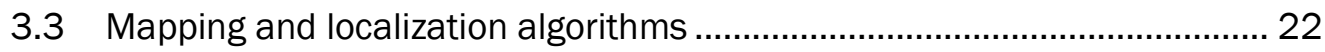

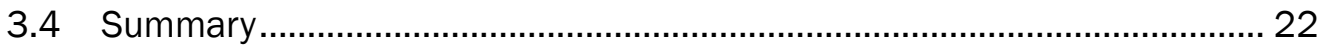

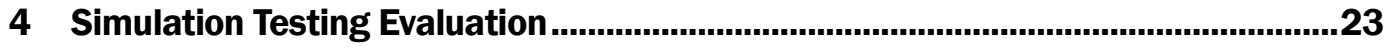

4.1 Evaluated SLAM algorithms ............................................................. 23

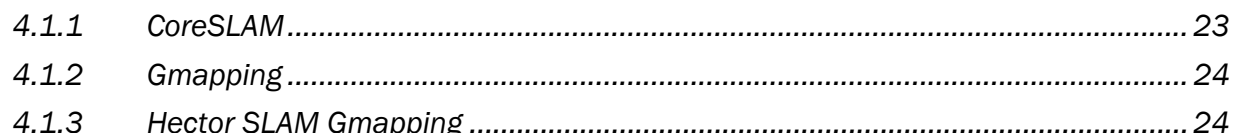




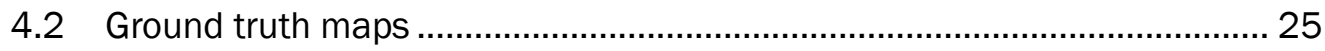

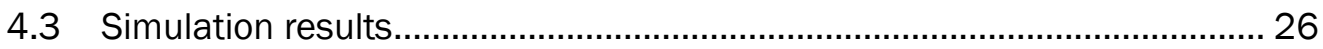

4.4 Image registration of ground truth and generated maps...........................27

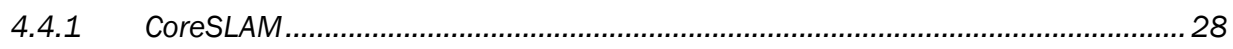

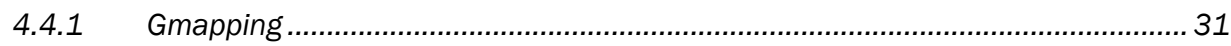

4.4.2 Hector SLAM .......................................................................................... 33

4.4.3 Comparisons of ground truth maps and Stage 4.1.1 generated maps................. 35

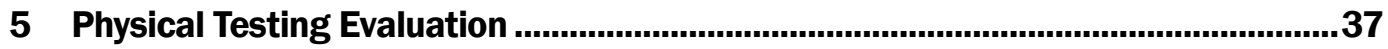

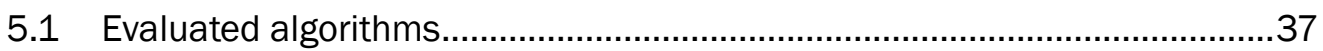

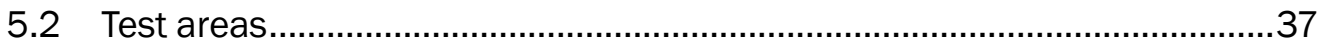

5.3 Generated maps from physical testing .................................................. 40

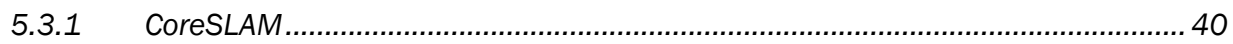

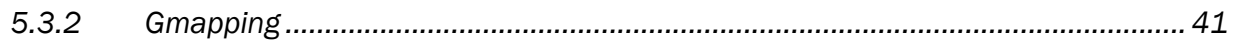

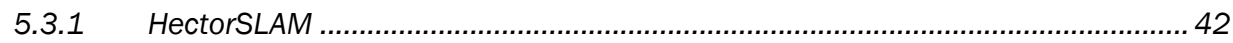

5.4 Comparison of ground truth map and generated map ............................. 42

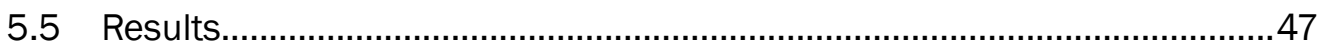

6 Localization and Pathfinding...........................................................................48

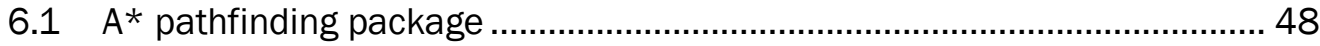

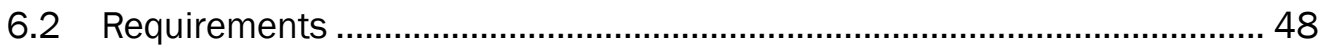

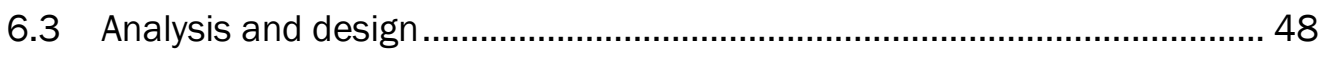

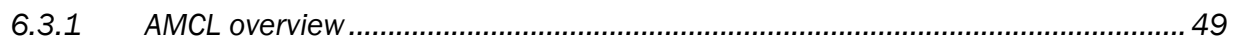

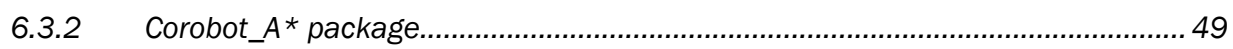

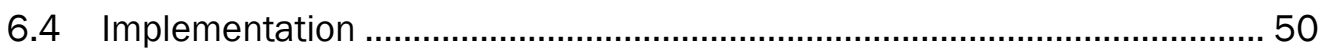

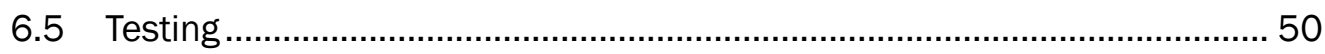

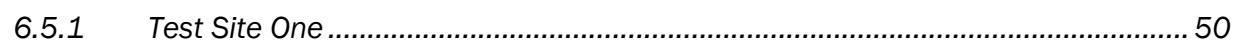

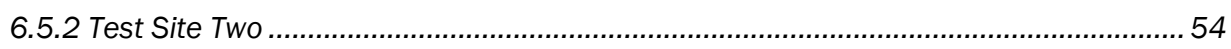

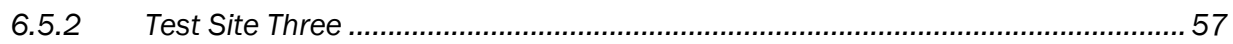

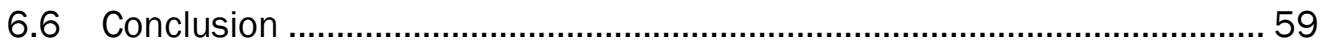

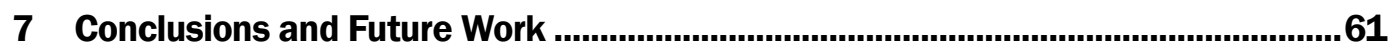

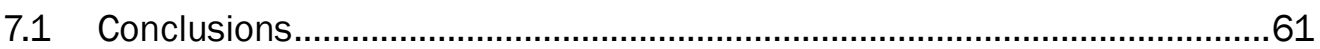

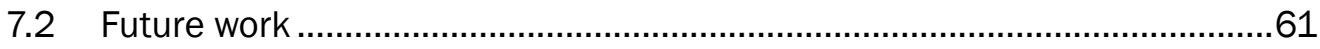

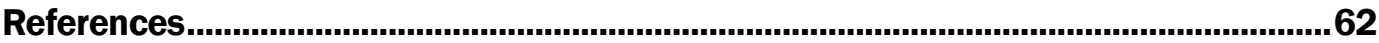

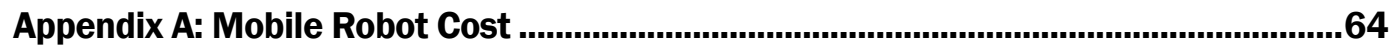




\section{Figures and Tables}

\section{Figures}

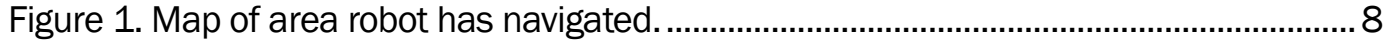

Figure 2. Monte Carlo Localization algorithms. ...............................................................11

Figure 3. Markov Localization algorithm (Fox et al. 1999)..................................................13

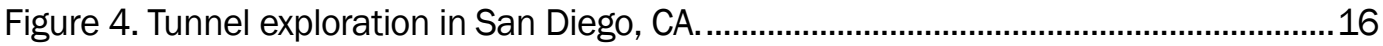

Figure 5. ALFUS defined level of autonomy.................................................................. 17

Figure 6. Superdroid Robots HD2 Treaded ATR Tank Robot kit........................................19

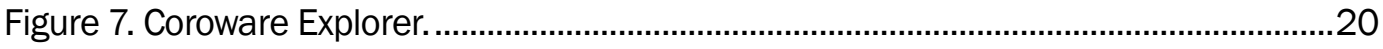

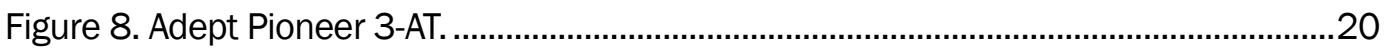

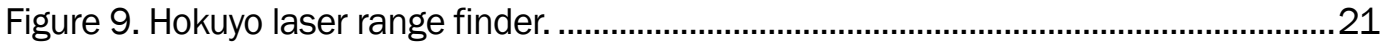

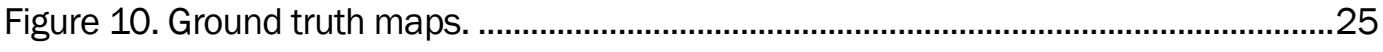

Figure 11. Map One and generated map of each algorithm..............................................2

Figure 12. Map Two and generated map of each algorithm. .............................................2

Figure 13. Map Three and generated map of each algorithm...........................................2

Figure 14. Image registration of Map One and CoreSLAM generated map........................29

Figure 15. Image registration of Map Two and CoreSLAM generated map..........................30

Figure 16. Image registration of Map Three and CoreSLAM generated map. ...................30

Figure 17. Image registration of Map One and Gmapping generated map.........................31

Figure 18. Image registration of Map Two and Gmapping generated map..........................32

Figure 19. Image registration of Map Three and Gmapping generated map. .....................32

Figure 20. Image registration of Map One and Hector SLAM generated map....................33

Figure 21. Image registration Map Two and Hector SLAM generated map.........................34

Figure 22. Image registration of Map Three and Hector SLAM generated map.................34

Figure 23. Ground truth map of foyer and break room. ......................................................38

Figure 24. Ground truth map of basement. ......................................................................39

Figure 25. Generated CoreSLAM map of basement. ...........................................................40

Figure 26. Generated CoreSLAM map of foyer. ....................................................................... 41

Figure 27. Generated Gmapping map of basement. ..............................................................42

Figure 28. Generated Gmapping SLAM map of foyer.....................................................43

Figure 29. Generated Hector SLAM map of basement. .......................................................44

Figure 30. Generated Hector SLAM map of foyer..............................................................45

Figure 31. Generated Hector Slam map and pictures of actual foyer. ................................46

Figure 32. Generated Hector Slam map and pictures of actual basement. ......................46

Figure 33. Test Site One with cones denoting points A and B...........................................51

Figure 34. Test Site One without cones and the SLAM generated map. .............................51

Figure 35. GIMP view with imported SLAM map...............................................................52 
Figure 36. Front and rear view of Test Site Two with cones.............................................55

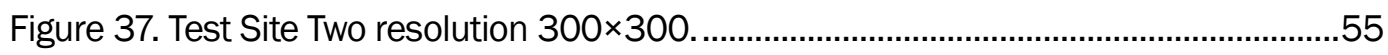

Figure 38. Test Site Three with cone............................................................................ 57

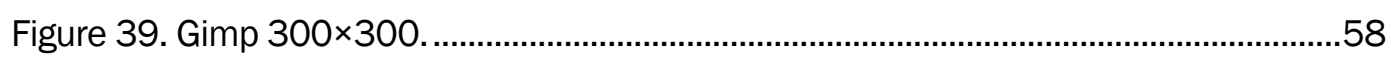

\section{Tables}

Table 1. Hausdorff distance for Map One. ...........................................................................36

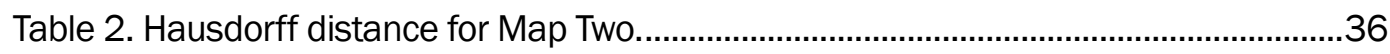

Table 3. Hausdorff distance for Map Three. ........................................................................36

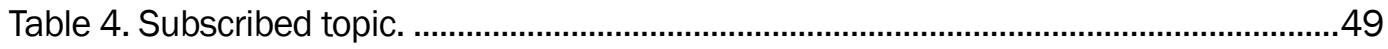

Table 5. Results for $100 \times 100$ on Test Site One. ...............................................................53

Table 6. Results $200 \times 200$ on Test Site One....................................................................53

Table 7. Results for $300 \times 300$ on Test Site One................................................................ 54

Table 8. Results for $500 \times 500$ on Test Site One................................................................54

Table 9. Results for $200 \times 200$ on Test Site Two...............................................................56

Table 10.Results for $300 \times 300$ on Test Site Two. ..................................................................56

Table 11. Results for $500 \times 500$ on Test Site Two..............................................................56

Table 12. Results for $200 \times 200$ on Test Site Three...........................................................59

Table 13. Results for $300 \times 300$ Test Site Three. ..............................................................59

Table 14. Results for $500 \times 500$ Test Site Three. ….........................................................59 


\section{Preface}

The work was conducted for the Deployable Force Protection and Force Protection Basing programs.

This work was performed by the Mobility Systems Branch (GMM) of the Engineering Systems and Materials Division (GM), U.S. Army Engineer Research and Development Center, Geotechnical and Structures Laboraory (ERDC-GSL). At the time of publication, Mr. Jeff Durst was Chief, CEERD-GMM; Dr. Gordon W. McMahon was Chief, CEERD-GM; and Ms. Pamela G. Kinnebrew, CEERD-GZT, was the Technical Director for Miliary Engineering. The Deputy Director of ERDC-GSL was Dr. William P. Grogan, and the Director was Mr. Bartley P. Durst.

COL Ivan P. Beckman was the Commander of ERDC, and Dr. David W. Pittman was the Director. 


\section{Introduction}

\subsection{Background}

Congress mandated that one-third of military vehicles be autonomous by 2015 (Kinney et al. 2006). Autonomy, in this case, implies that the unmanned ground vehicle (UGV) should be able to traverse a dynamic and unstructured environment with little or no human intervention. In the broadest sense, a UGV is any piece of mechanized equipment that moves across the surface of the ground and serves as a means of carrying or transporting cargo, but explicitly does NOT carry a human being (Gage 1995). The military uses the UGV to perform reconnaissance and surveillance on the battlefield and in urban settings, tunnels, and other military missions. There exists a limited number of available robotic platforms and architectures equipped to navigate and perform mapping and localization of complex environments.

The goal of this research is to develop a software system to support UGVs exploration complex environments of tunnels and other subterranean areas such as basements, old mines, sewers, and caves. The Department of Defense (DoD) has used robotic platforms for various missions over the past 10 years. The Department of Homeland Security (DHS) uses robotic platforms in exploring drug tunnels along the southern U.S. border and Mexico (see examples below). The development of a robust framework for autonomous UGVs will lead to an increase in the availability of robotic platforms and architectures to the DoD and DHS through the use of modeling and simulation.

September 11, 2001, was a very devastating day, one that forever changed the United States of America (USA). Terrorist attacks occurred at the World Trade Center (WTC) towers in New York City and at the Pentagon in Washington, D.C. Tele-operated robots were used under the direction of the Center for Robot-Assisted Search and Rescue from September 11 to October 2, 2001, to search for victims and to help assess the structural integrity of the WTC foundation (Murphy 2004). The robots were used for tasks that the rescuers or canines could not perform; for example, to either go into spaces too small for a human or to pass through an area still burning (Murphy 2004). Before September 11, 2001, the Oklahoma City bombing on April 19, 1995, motivated an interest in the domain of rescue 
robotics for urban search and rescue (Murphy 2004). Robin R. Murphy states that urban search and rescue (USAR) missions, which deal with man-made structures, have a different emphasis than traditional wilderness rescue or underwater recovery efforts and can be even more demanding on robot hardware and software design than military applications (Murphy 2004).

During the search and rescue phase after September 11, 2001, the insertion of the Inuktum micro-VGTV robot into a sewer pipe at the WTC site allowed rescuers to attempt to locate an entry into a basement (Murphy 2004). The robot was small in size and tethered, using a safety line for vertical entry (Murphy 2004).

On March 20, 2003, the USA invaded Iraq, a war different from any other because of new technological advances. One such advancement was the Dragon Runner Reconnaissance Robot, developed by the National Robotics Engineering Centre (NREC). Weighing about $15 \mathrm{lb}$, the Dragon Runner was designed to be light enough to toss into a window or up and down stairs (Voth 2004). The U.S. Marine Corps used the Dragon Runner during Operation Iraqi Freedom with approximately a dozen deployed for sentry missions and urban reconnaissance (Voth 2004). When U.S. forces went into Iraq in 2003, they had a limited number of robotic units on the ground (Singer 2008). By the end of 2004, the number was up to 150 (Singer 2008). By the end of 2005, it was up to 2,400, and it more than doubled the next year (Singer 2008).

Since 1990, approximately 130 tunnels have been discovered along the border between San Diego, CA, and Tijuana, Mexico; one of the more recent discoveries was on March 16, 2016. According to the New York Daily News article by Alfred Ng, federal agents apprehended a 415-yard tunnel between Mexicali, Mexico, and Calexico, CA, after a 16-month investigation, seizing almost 3,00o lb of marijuana. The tunnel had entrances at a restaurant in Mexico and a newly built house in California. From 2010 to date, the tunnels have become more sophisticated, spacious, and expensive. According to Alfred Ng, the March 16, 2016, tunnel is the first instance where drug traffickers bought property in the U.S. specifically for hiding a drug tunnel.

According to the Associated Press article by Elliot Spagat on November 27, 2010, the sophisticated cross-border tunnel, equipped with a rail system, 
ventilation, and fluorescent lighting, was the second discovery of a major underground drug passage in San Diego during November 2010. The U.S. Homeland Security and Mexican officials shut down this tunnel. The elaborate tunnel's length was 2,200 ft. The tunnel was between the kitchen of a home in Tijuana, Mexico, and two warehouses in San Diego's Otay Mesa industrial district. The cost of constructing these tunnels appears to be enormous because of the sophistication of their design. The first tunnel, discovered on November 3, 2010, spanned 600 yd and contained 25 tons of marijuana. Discovery of these tunnels has escalated in the past three years, along with border security efforts such as border fence installations, increased border agents, and the call for DoD support from the DHS. The discovery of these tunnels greatly concerns both the DoD and the DHS. Both agencies have identified a requirement for methods that will easily explore and map out the unknown tunnel's environments without sending humans into the tunnels and endangering them. Developing robotic platforms to perform this task would mitigate the danger to humans.

\subsection{Types of unmanned vehicles}

The USA took a variety of vehicles to war; however, basically three distinct types of unmanned vehicles were used: (1) unmanned ground vehicles (UGV), (2) unmanned aerial vehicles (UAV), and (3) autonomous underwater vehicles (AUV). Each of these unmanned vehicles saw action in the Iraqi War, and some were for tunnel exploration along the United StatesMexico border.

\subsubsection{Unmanned ground vehicles}

The U.S. Army uses two major types of autonomous and semi-autonomous ground vehicles: large vehicles and small vehicles. Examples of large vehicles are tanks, trucks, and high-mobility, multiple-wheeled vehicles also known as the HUMVEEs. Examples of small vehicles, sized for carrying by a soldier in a backpack, are the PackBot and Talon. The PackBot and Talon move around on treads like small tanks.

Manufactured and sold by the iRobot Corporation of Burlington, MA, the PackBot weighs approximately $28 \mathrm{~kg}(61.78 \mathrm{lb})$ and costs approximately $\$ 40,000$. The PackBot performed reconnaissance and neutralization of unexploded ordnance (UXO) and improvised explosive device sites, which posed a major problem to the U.S. Military during the Iraqi War. 
Manufactured and sold by Foster-Miller Inc., the Talon weighs approximately $38 \mathrm{~kg}(85 \mathrm{lb})$ and costs approximately $\$ 60,000$. The Talon performs a variety of functions from reconnaissance to weapons delivery. Equipped with a robotic arm, uses of the Talon include surveillance or grabbing. The Talon performed search and recovery missions at the World Trade Center after the September 11 attack and various missions in the war in Afghanistan (Voth 2004). Twenty Talon robots were deployed in Iraq in the beginning of 2003 and have accomplished approximately 10,000 or more missions (Voth 2004).

\subsubsection{Unmanned aerial vehicles}

The UAV is an unmanned aerial vehicle; for example, a drone. The military has been the dominant customer for UAVs in the United States, spending nearly half a billion dollars annually on UAVs in recent years (Russell and Norvig 1995). The organizations and tactical units within the Office of the Secretary of Defense, Army, Navy, Marine Corps, and Air Force are responsible for specifying, acquiring, and operating UAVs (Russell and Norvig 1995). Examples of UAVs are drones and the MQ-9 Reaper.

\subsubsection{Autonomous underwater vehicles}

An AUV is an autonomous underwater vehicle. The main fields of AUV application include: (1) ocean exploration and monitoring of water medium, (2) marine geological survey, (3) inspection of the underwater engineering structures and pipelines, (4) search, inspection, and rescue operations, and (5) protection of environment and maricultures (Russell and Norvig 1995). AUVs were used to search 3.5 million square meters of shallow water for mines in Operation Enduring Freedom in Iraq in 2003 (Edwards et al. 2004). Examples of AUVs are the Autonomous Benthic Explorer and the Odyssey IV.

\subsection{Proposed research}

Designed for relatively benign environments related to terrain, command and control, tethered robotic platforms such as pipe inspections systems, surveillance robots, explosive ordnance disposal robots, and mine clearance robots perform at a low level of autonomy (Doray et al. 2009). In contrast, a tunnel environment presents several challenges to a UGV: i.e., the complexity of the terrain, communications limitations (GPS does not 
work underground), and limited amounts of pre-existing data about the tunnel's environment.

\subsection{Objective}

This dissertation research focuses on building a software system to support UGVs in exploring tunnels. The goal of this research is to build a robust architecture to perform tunnel mapping and localization in an unknown environment, hence to increase the level of autonomy of a UGV. The current UGV designs cannot accomplish the functions such as localization and mapping to operate in tunnel environments while communicating with aboveground systems.

\subsection{Organization of report}

Chapter 2 provides background information on current and past research on localization and mapping UGVs in varied environments. Chapter 3 provides information on the robotic platform used in this research, the software, and the selection of algorithms for localization and mapping in the prototype system. Chapter 4 provides details and the results of the simulation experiments using CoreSLAM, Gmapping, and HectorSLAM. Chapter 5 provides details and the results of the physical testing experiments using CoreSLAM, Gmapping, and HectorSLAM field-testing for the robotic platform. Chapter 6 provides details on localizing the robotic platform to maps of various resolution generated by HectorSLAM, pathfinding with $\mathrm{A}^{*}$, path traversal with the Explorer localized to the generated map. Chapter 7 provides conclusions and future work. 


\section{Literature Review}

\subsection{Introduction}

This chapter provides an overview of research in the area of localization and mapping of robots in uncertain complex environments such as buildings, urban areas, mines, underwater, and tunnels. Also included is an overview of past and current research by the Engineering Research and Development Center (ERDC) on modeling and simulation and tunnel exploration.

\subsection{Mapping}

Robotic mapping addresses the problem of acquiring spatial models of physical environments through mobile robots (Thrun 2002). Tasks performed by the robot include the identification of features, such as landmarks, distinctive objects, or shapes and estimation of the robot's location in reference to the identified features. One of the fundamental tasks in robotics is the creation of a map of the area where the robot is moving (Rozman 2009). The robot uses the created map for its navigation in this environment.

The robot-mapping problem, more specifically, consists of the robot finding its pose, creating a map, and integrating the two. The robot has to keep track of its state based on data perceived from its sensors. The pose, the most important part of the state, provides information related to the location and orientation of the robot relative to its environment, i.e., pose $=(x, y, \theta)$, where $x$ and $y$ represent the location and $\theta$ is the orientation of the robot relative to a given coordinate frame.

\subsubsection{Robotic mapping problems}

Sebastian Thrun lists the five following problems associated with robotic mapping: (1) measured noise, (2) map size, (3) correspondence/data association problem, (4) dynamic environments, and (5) robotic exploration (Thrun 2002b).

As discussed earlier, robots, equipped with varied sensors to perceive their environment, navigate their environment seeking landmarks. As the robot navigates its environment, the sensor data may contain errors in measuring its environment. The errors are also defined as noise. The cause 
of the errors may be slippage caused by odometry errors or sensor noises because of real-world predicaments. Odometry errors will accumulate and throw off an entire map.

Second, there is the problem of the size of the map. The size of the map may be increased as the robot navigates. When mapping a robot's environment, the information about the environment has to be stored. Storing the map requires more memory space and computational time as the map increases in size.

The third and possibly hardest problem in robotic mapping is the correspondence problem, also known as the data association problem. The data association problem is associated with differentiating between sensor measurements taken at time $t$ corresponding with the same physical object in the world. The correspondence problem determines if two data points taken from different scans are the same object.

The fourth problem, dynamic environment, deals with environmental change as time passes, e.g., a landmark that is a moving object such as a person. Changes in an environment can be from slow to fast.

The final problem is robotic exploration during mapping, the task of generating robot motion in the pursuit of building a map. Figure 1 depicts a map of an UGV while exploring a tunnel and the map that it creates during the exploration of the tunnel.

\subsubsection{Metric and topological maps}

According to the type of maps generated, mapping algorithms can be roughly divided into two categories: metric approaches and topological approaches. The first approach models the environment using a metric map, enabling accurate estimation of the robot's positions. A metric approach typically provides a dense representation of the environment; therefore, it is well suited to precise trajectory planning (Angeli et al. 2008). In a topological approach, the environment, segmented into distinctive pieces, forms the nodes of a graph or topological map. The neighboring relations (i.e., whether or not a piece is accessible from another one) is modeled using the edges of this graph (Angeli et al. 2008). Topological mapping relies on a higher level of representation than metric mapping, allowing symbolic goal-driven planning and navigation. 
Figure 1. Map of area robot has navigated.

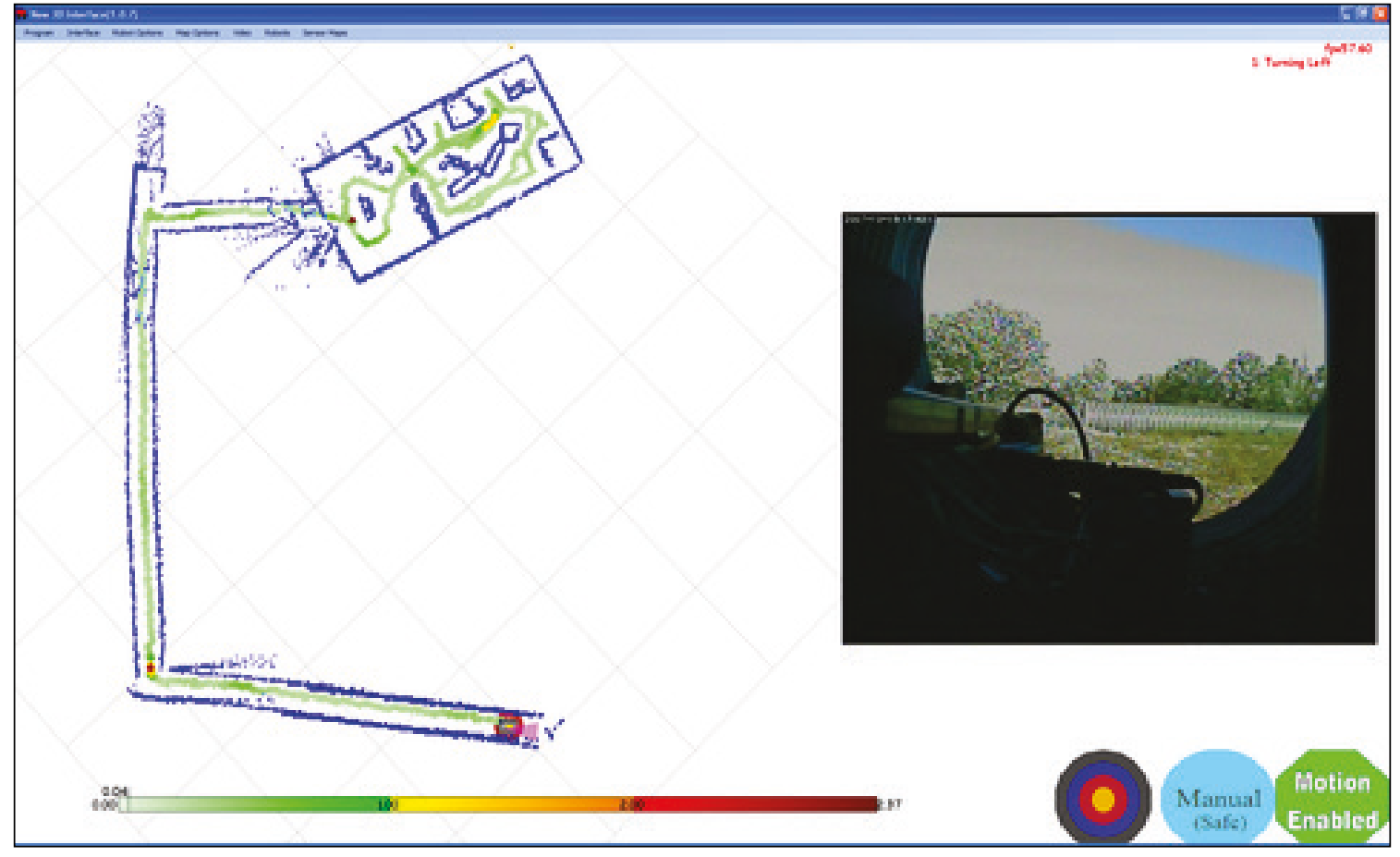

Compared with metric mapping, topological mapping usually provides a more compact representation that scales better with the size of the environment.

\subsubsection{Probabilistic mapping algorithms}

The correspondence problem is the key to solving either the metric mapping or topological mapping problem, i.e., the robot must be able to determine if data taken at different times correspond to the same physical object (Reynolds 2005). In this regard, probabilistic techniques yield some of the most accurate results of any of the methods.

At the foundation of any probabilistic algorithm for robotic mapping lies Bayes' Rule (1), where $x$ represents the map and $d$ represents the data for the sensors. $\operatorname{Pr}(x)$ is the prior probability of the map, and $\operatorname{Pr}(x \mid d)$ is the probability of the map $\mathrm{x}$ is true given the sensor measurement $d$, and $\mathrm{p}(d \mid x)$ is the probability of the sensor measurement being $d$ given an object at $x$.

$$
\operatorname{Pr}(\mathrm{x} \mid \mathrm{d})=\frac{p(d \mid x) \operatorname{Pr}(x)}{p(d)}
$$


Usually, a Bayes estimator approximates both the map and the robot's pose.

The correspondence problem also can be tackled in an incremental fashion. For example, Reynolds (2005) discussed a maximum likelihood approach that compares nearby measurements of the previous map to identify the path the robot has moved within a small time frame. This approach was shown to be robust in the sense that it can recover from a wrong correspondence. Nevertheless, it takes a large amount of processing, making it less ideal for real-time applications.

An occupancy grid is one of the most popular incremental algorithms because of its ease of use and robustness. In the most basic form, the binary occupancy of a location $(x, y)$ is calculated and the cells (grids) are incrementally updated (Reynolds 2005). This approach works well in a real-time application; however, it relies heavily on odometry data. This means that the errors in odometry data will accumulate and, hence, skew the map.

The above error accumulation problem can be alleviated by a hybrid method, e.g., combining a Bayes estimator with an incremental algorithm. Although they are typically more difficult to implement, they provide significantly better results than either a purely Bayes estimator or an incremental method for autonomous robots (Reynolds 2005).

\subsection{Localization}

Localization refers to the estimation of the position of a mobile robot on a known or a predicted map (Stanculescu and Sojka 2008). It requires using a map to interpret sensor data to determine the configuration of the robot. Without the ability to localize itself in an environment successfully, a robot is effectively stripped of its ability to do useful work (Kramer 2010).

There are three issues involved with localization: the local position tracking problem, the global position tracking problem, and the kidnapped robot problem. Each problem has been extensively researched in the literature.

The local position tracking problem has received the most attention. When dealing with this problem, the robots initial pose is known. For global position tracking, the robot is unaware of its initial pose and has to 
determine its location from scratch. In the kidnapped robot problem, the robot knows its location; however, it is relocated and is totally unaware of its new location. The mobile robot must figure out its location on its own completely. This problem is used to test a robot's ability to localize itself.

Although there are many methods for determining the location of the robot, in this research three methods are discussed in detail as follows: (1) Monte Carlo Localization, (2) Kalman filters, and (3) Markov Localization. Next, we provide a summary of which of the three localization problems these methods solve, along with one other possible solution to both the localization and mapping problems.

\subsubsection{Monte Carlo Localization}

Monte Carlo Localization (MCL) has been widely used to estimate a robot's pose and to solve the global localization problem, in which the robot does not know its starting position (Thrun et al. 2001). MCL consists of four steps: sampling, prediction, update, and weight normalization as depicted in Figure 2 below. The general idea is as follows.

1. Initialize a set of samples (the current samples) so that their locations are evenly distributed and their importance weights are equal.

2. Repeat until done (i.e., weights converge) with the current set of samples:

a. Move the robot a fixed distance and take a sensor reading.

b. Update the location of each of the samples (using the movement model).

c. Assign the importance weights of each sample to the likelihood of that sensor reading given that new location (using the sensor model).

d. Create a new collection of samples by sampling (with replacement) from the current set of samples based on their importance weights.

e. Let this new collection be the current set of samples.

Research performed by Stanculescu and Sojka (2008) evaluates the Monte Carlo Localization algorithm. It was concluded that MCL efficiently estimates the position of a robot on a grid-based map. 
Figure 2. Monte Carlo Localization algorithms.

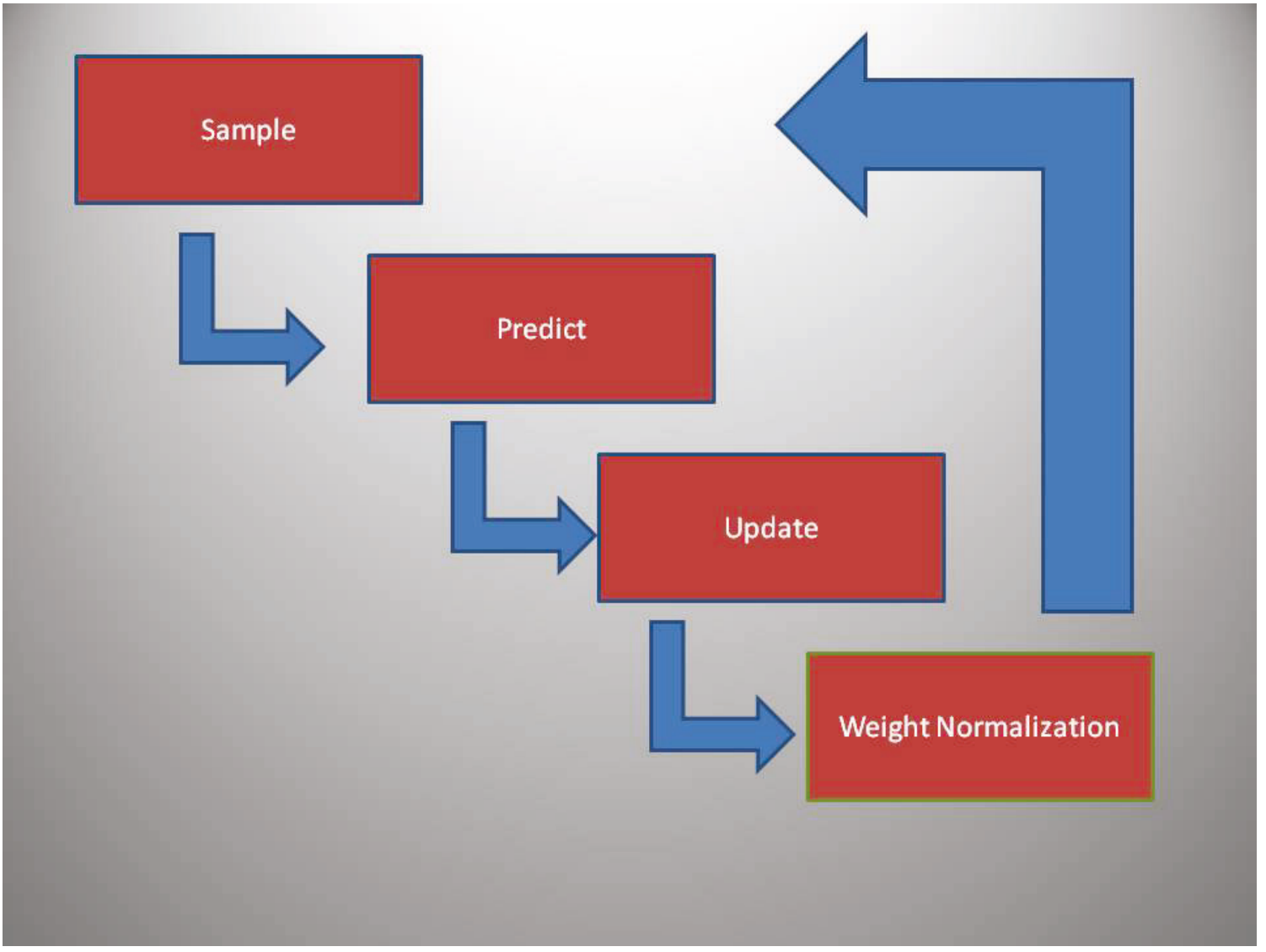

In Dellaert et al. (1999), the researchers introduce a MCL method that represents the probability density involved by maintaining a set of randomly drawn samples. By using a sampling-based representation, they obtain a localization method capable of representing an arbitrary distribution. Experimentally, they show that the resulting method can efficiently localize a mobile robot without knowledge of its starting position, which is faster, more accurate, and less memory-intensive than earlier grid-based methods.

One advantage of using the MCL is its ability to represent multi-modal distributions; hence, it can be used to globally localize a mobile robot. A second advantage is that MCL drastically reduces the amount of memory required compared to a grid-based Markov Localization (discussed in Section 2.3.3) and can integrate measurements at a considerably higher frequency. A third advantage is that, like many randomized algorithms, it is easy to implement (Stanculescu and Sojka 2008). 
Many variants of the MCL method exist, one such method is Mixture Monte Carlo Localization, a mobile robot localization algorithm. It is a version of particle filtering that combines a regular sampler with its dual (Thrun et al. 2001). The Mixture-MCL provides efficiency, versatility, resource adaptiveness, and robustness.

\subsubsection{Kalman filters}

In a seminal paper, R.E. Kalman (1960) developed a probabilistic filtering algorithm for a control system. The Kalman filter has been widely applied in robot perception. Its purpose is to use sensor measurements observed over time, containing noise (random variation) and other inaccuracies, to produce values that tend to be closer to the true values of the measurements than the observed values.

Kalman filters are essentially Bayes' filters under a Gaussian assumption. They have been widely applied to estimate the robot's pose. The Kalman filter algorithm operates on the postulation that the current state function must be a linear step from the previous state function with Gaussian noise. The Kalman filter is an effective approach in several aspects: (1) it supports estimations of past, present, and even future states, and (2) it can do so even when the precise nature of the modeled system is unknown (Welch and Bishop 1995).

Although Kalman filter-based techniques have proven to be robust and accurate for keeping track of the robot's position, it relies on the Gaussian assumption and lacks the ability to globally (re-)localize the robot in the case of localization failures (Dellaert et al. 1999). Although the Kalman filter can be amended in various ways to cope with some of these difficulties, recent approaches have used richer schemes to represent uncertainty, moving away from the restricted Gaussian density assumption inherent in the Kalman filter (Dellaert et al. 1999).

\subsubsection{Markov Localization}

Markov Localization is a special case of probabilistic state estimation using Bayes' rule. It is robust against both inaccurate maps and noisy sensors (Song 2002). In many probabilistic mobile robot localization literatures, the term Markov Localization and Bayesian estimation (filter) are used interchangeably. Markov Localization uses a probabilistic framework to maintain a position probability density over the whole set of possible robot 
poses. It is based on the Markov Assumption, i.e., the environment is static (Fox et al. 1999). Instead of maintaining one hypothesis as to where in the world a robot may be, it maintains a probability distribution over the space of all such hypotheses (Fox et al. 1999). The probabilistic representation allows it to weigh these different hypotheses in a mathematically sound way (Fox et al. 1999). The pseudo code of a general Markov Localization algorithm is given in Figure 3.

Figure 3. Markov Localization algorithm (Fox et al. 1999).

for each location $l$ do /*initialize the belief */

$$
B \in l_{n}(l) \leftarrow-P\left(L_{n}^{(0)}=l\right)
$$

end for

forever do

if the robot receives new sensory input $a_{n}$ do

for each location $l$ do

/* apply the perception model */

end for

$$
B \in l_{n}(l) \leftarrow-\alpha P\left(o_{n} \mid l\right) B \in l_{n}(l)
$$

end if

if the robot receives a new odometry reading $a_{n}$ do

for each location $l$ do

/* apply the motion model */

end for

$$
B \in l_{n}(l) \leftarrow-\int P\left(l \mid a_{n}, l^{\prime}\right) B \in l_{n}\left(l^{\prime}\right) d l^{\prime}
$$

end if

if the robot is detected by the $m$-th robot do

for each location $l$ do

/* apply the detection model $* /$

$$
B \epsilon l_{n}(l) \leftarrow-B \epsilon l_{n}(l) \int P\left(L_{n}=l \mid L_{m}=l^{\prime}, r_{m}\right) B \epsilon l_{m}\left(l^{\prime}\right) d l^{\prime}
$$

end for

end if

end forever

Markov Localization has produced excellent success in mobile research areas, such as working as guidance robots at a crowded museum. Minerva and Rhino are two mobile robots using different types of robots and sensor modalities to act as interactive museum tour-guides. The major advantage of Markov Localization is its ability to detect localization failures and to relocalize the robot (Fox et al. 1999). The only disadvantage lies in the fixed representation of the grid, which has an undesirable effect; the memory 
requirement stays constant even if only a minor part of state space is updated.

\subsection{Simultaneous localization and mapping}

Simultaneous localization and mapping (SLAM) is a technique used by robots and autonomous vehicles to build a map within an unknown environment (without apriori knowledge) or to update a map within a known environment (with apriori knowledge from a given map) while keeping track of their current location. SLAM, which was introduced by R. Smith, M. Self, and P. Cheeseman in 1990, laid the groundwork for the modern SLAM problem using Extended Kalman Filters.

A solution to the SLAM problem has been seen as the "Holy Grail" and would enable robots to operate in an environment without a priori knowledge of obstacle locations. The characteristics of SLAM couple the problems of localization and mapping. The two quantities are to be inferred from a single measurement. Loosely speaking, a SLAM process consists of multiple steps: landmark extraction, data association, state estimation, state update, and landmark update (Riisgaard and Blas 2004).

To achieve SLAM, two models are required, the process model and the observation model. In the process model, a vehicle traverses through an environment containing a population of landmarks with a known kinetic model. In the observation model, the vehicle is equipped with a sensor that can take measurements of the relative location between any individual landmark and the vehicle itself. Various versions of SLAM exist: FastSLAM 2.0, Marginal SLAM, and EKF -SLAM, to name a few.

\subsection{Research goal}

Currently, a very limited number of robotic platforms are available for tunnel exploration. The goal of this research is to develop a software platform to support a UGV to perform localization and mapping of a tunnel. The robotic platform should be able to detect humans and objects while exploring the tunnel. This research aids in expanding the number of robotic platforms available for use by the DoD and the DHS. Also, another goal is to increase the level of autonomy for UGVs in which the ERDC currently use from tethered UGV. Next, we discuss the modeling and simulation, tunnel exploration, and levels of autonomy. 


\subsubsection{Modeling and simulation}

Modeling and simulation (M\&S) is a tool that saves time, money, and lives. M\&S has been used by the military since the early 1940s. According to the Department of Defense's Modeling and Simulation Primer, military analysts use $M \& S$ to help shape the size, composition, and structure of forces to meet national military requirements, and to assess the sufficiency of operational plans. The Primer also states that the military acquisition community uses M\&S (1) to evaluate requirements for new systems and equipment; (2) to conduct research, development, and analysis activities, (3) to develop digitized prototypes and avoid the building of costly full scale mockups, and (4) to plan for efficient production and sustainment of the new systems and equipment when employed in the field.

The Department of Defense's Modeling and Simulation Primer defines a model as a physical, mathematical, or logical representation of a system, entity, phenomenon, or process with no movement, as in a replica of a car or airplane. The model may take the form of a set of assumptions concerning the operation of the system (Banks and Carson 1984). Once developed and validated, a model can be used to investigate a wide variety of "what if" questions about the real-world system.

In Discrete-Event System Simulation, simulation is defined by Banks as the imitation of the operation of a real-world process or system over time. Whether done by hand or on a computer, simulation involves the generation of an artificial history of a system and the observation of that artificial history to draw inferences concerning the operation of characteristics of the real system (Banks and Carson 1984).

\subsubsection{Tunnel exploration}

The Unmanned Tunnel Exploration (UTE) research effort's goal is to develop a Semi-Autonomous Unmanned System for Small Unmanned Ground Vehicles in tunnel environments, and Intelligence, Surveillance, and Reconnaissance (ISR) processes (Doray et al. 2009). The UTE research effort combines high resolution vehicle simulations with field experiments to advance the operational capability of SUGV's operating in tunnels. The UTE technology will have a significant impact on successfully deploying the first generation Tunnel Activity Detection Systems (TADS) solution through the Joint Task Force-North (JTF-N), a subordinate command of US NORAD-NORTHCOM, and the US ARMY ERDC. Further collaboration 
is being conducted with TAE Technical Support Working Group, US ARMY TRADOC Analysis Center, Monterey, and Idaho National Labs. Figure 4 depicts the TALON navigating a tunnel in San Diego.

Figure 4. Tunnel exploration in San Diego, CA.

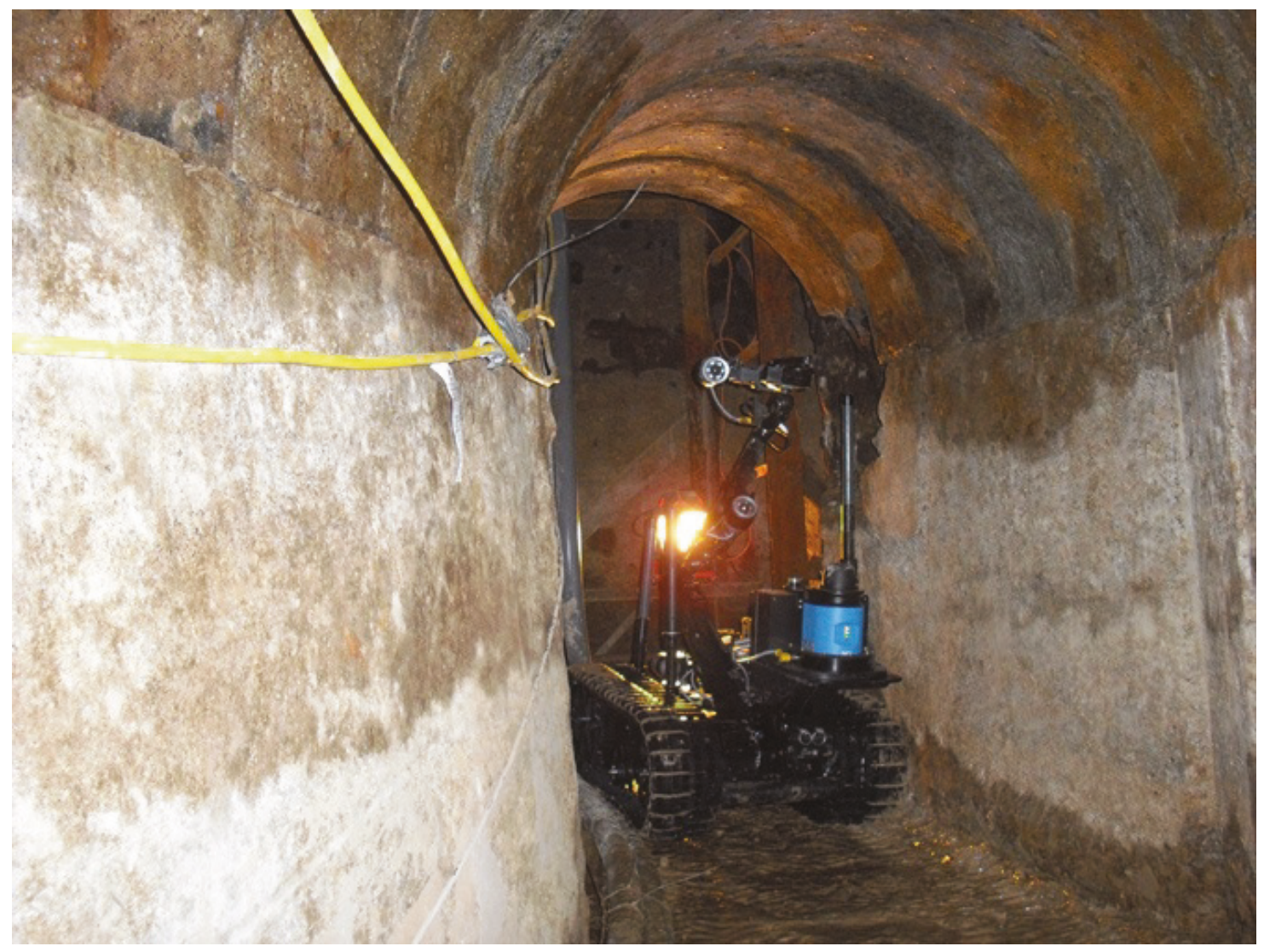

\subsubsection{Levels of autonomy}

An autonomous navigation system or autonomous robot can function, operate, or make decisions independently under reasonable circumstances, with limited human intervention. Increasing levels of human intervention decrease the autonomy of the navigation system. A classification of levels of autonomy takes into account the interaction between human control and the machine motions: teleoperation, supervisory, task-level autonomy, and full autonomy. In teleoperation, a human controls each movement. Each machine actuator change is specified by the operator. At the supervisory level, a human specifies general moves or position changes, and the machine decides specific movements of its actuators. At the task-level autonomy, the operator specifies only the task, and the robot manages itself to complete it. In full autonomy, the machine will create and complete all its tasks without human interaction. Generally, higher levels of autonomy do 
not necessarily require more complex cognitive capabilities. For example, robots in assembly plants are completely autonomous, but operate in a fixed pattern.

The Autonomy Levels of Unmanned Systems (ALFUS) working group's definition of levels of autonomy is displayed in Figure 5. The ALFUS level ranges from $o$ to 10 , where at $o$ there is no autonomy and the robot is controlled by remote control and at 10 the system is a fully intelligent system with no human interface.

Figure 5. ALFUS defined level of autonomy.

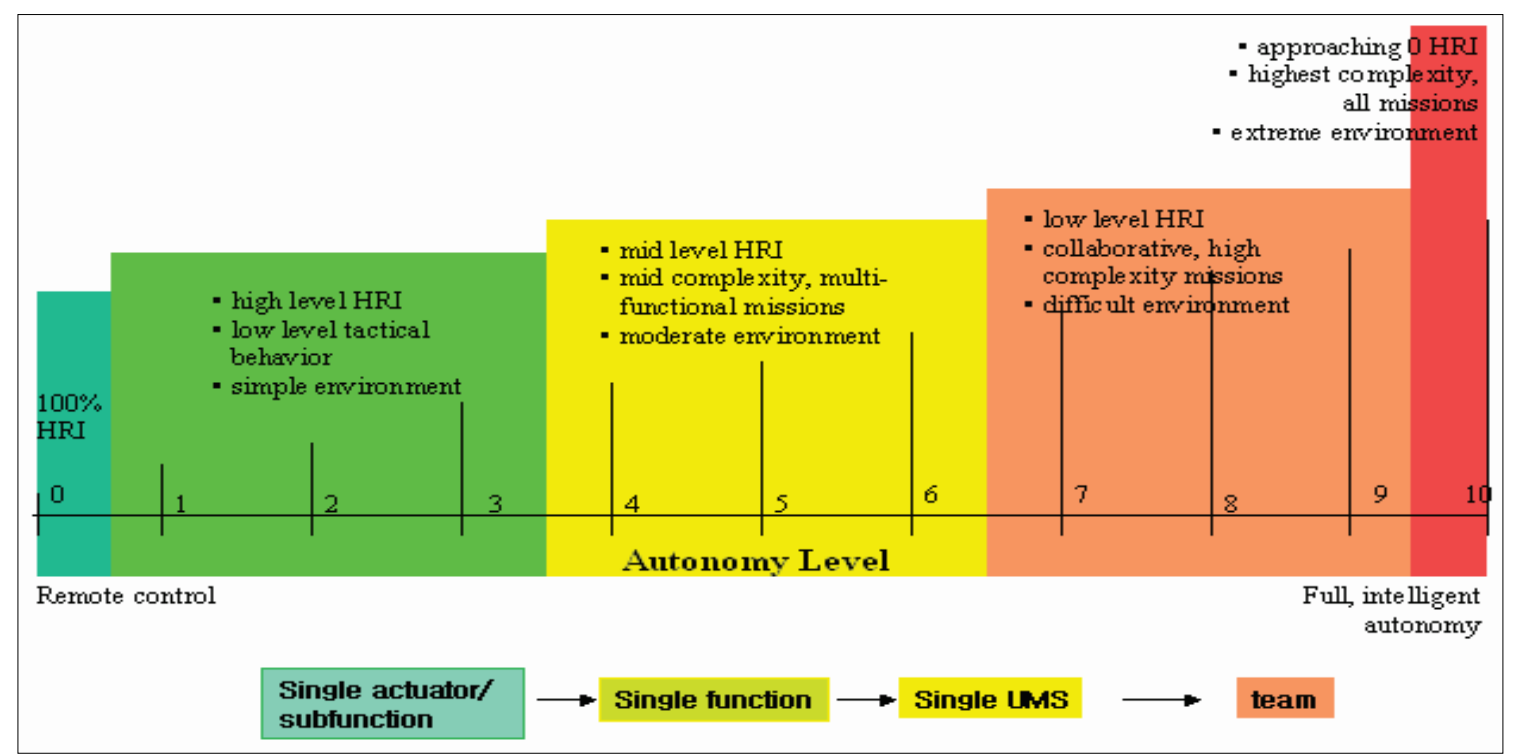

\subsection{Proposed research}

In this research, software was designed and tested for a UGV, the Coroware Explorer, to enable it to function as an UGV with the capability to localize and map an unknown area while navigating. The UGV was equipped with a camera, acoustic sensor, and a Laser Range Finder that provides the UGV with readings to determine its pose and landmarks within the unknown area to create a map of the unknown research.

The developed system was validated using simulation and field-testing to determine its ability to navigate the unknown environment, detect landmarks, and build a map of the navigated environment. Three scenarios were used for simulation testing. The simulated test environment was the STAGE simulator and the test sites were in facilities at the ERDC in Vicksburg, MS. 
This research uniquely combines Coroware Explorer and field-testing. In the next chapter, we introduce Coroware Explorer, the robot platform in the project. 


\section{Overview of System}

\subsection{Description of robot platform}

The following UGV platforms received consideration as possible platforms for this research: (1) the Adept Pioneer 3-AT, (2) the Coroware Explorer, and (3) Superdroid Robots HD2 Treaded ATR Tank Robot Kit. Each of these platforms is a programmable robot, easily adaptable to varied sensors. Figure 6 depicts the Superdroid Robots HD2 Treaded ATR Tank Robot Kit, Figure 7 depicts the Coroware Explorer, and Figure 8 depicts the Adept Pioneer 3-AT.

Figure 6. Superdroid Robots HD2 Treaded ATR Tank Robot kit.

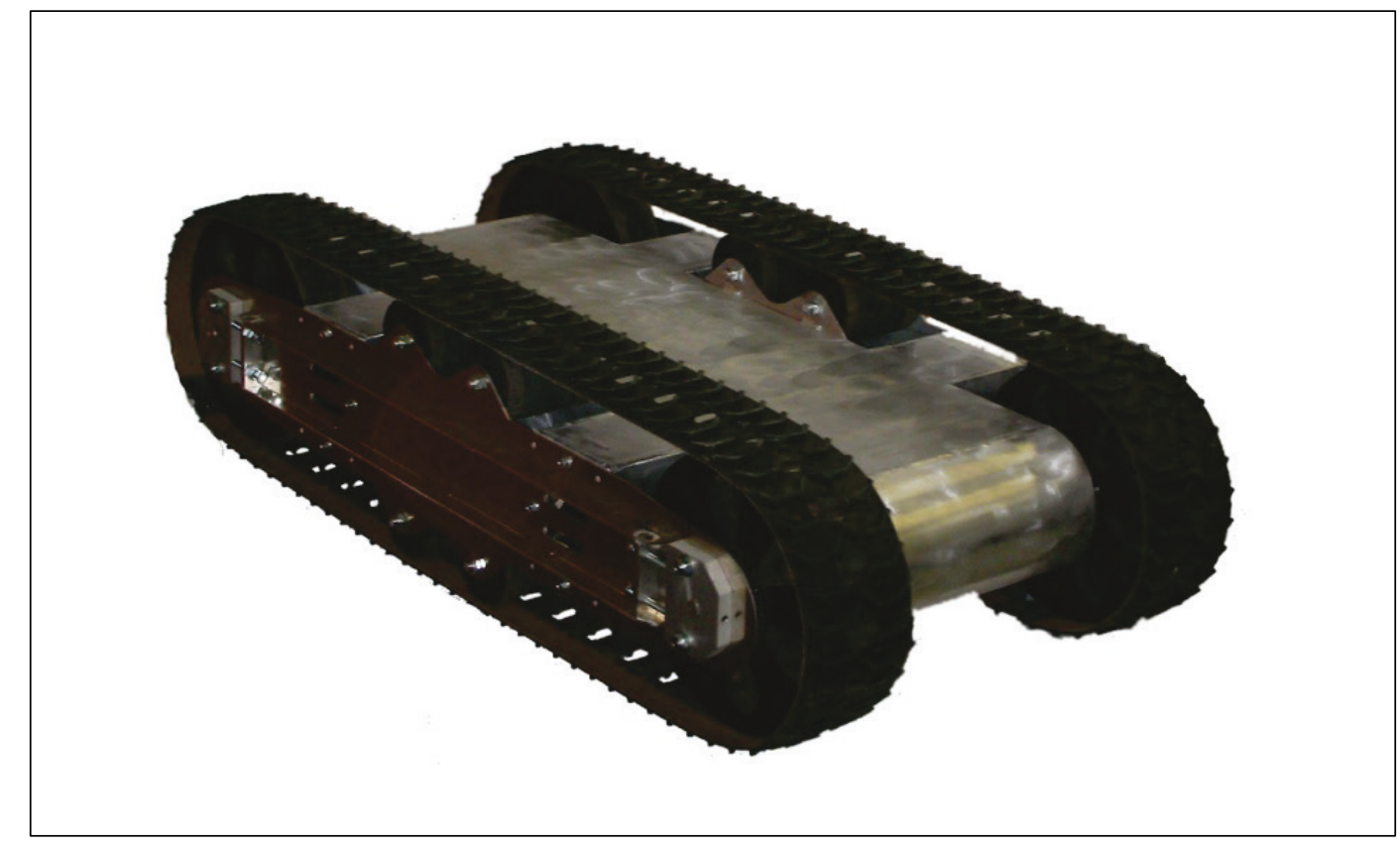

The selection of the robot is based on several requirements.

- It may be tracked or wheeled.

- It must be capable of crossing objects of various sizes such as rocks, water, and gravel.

- It must support a range of sensors. 
Figure 7. Coroware Explorer.

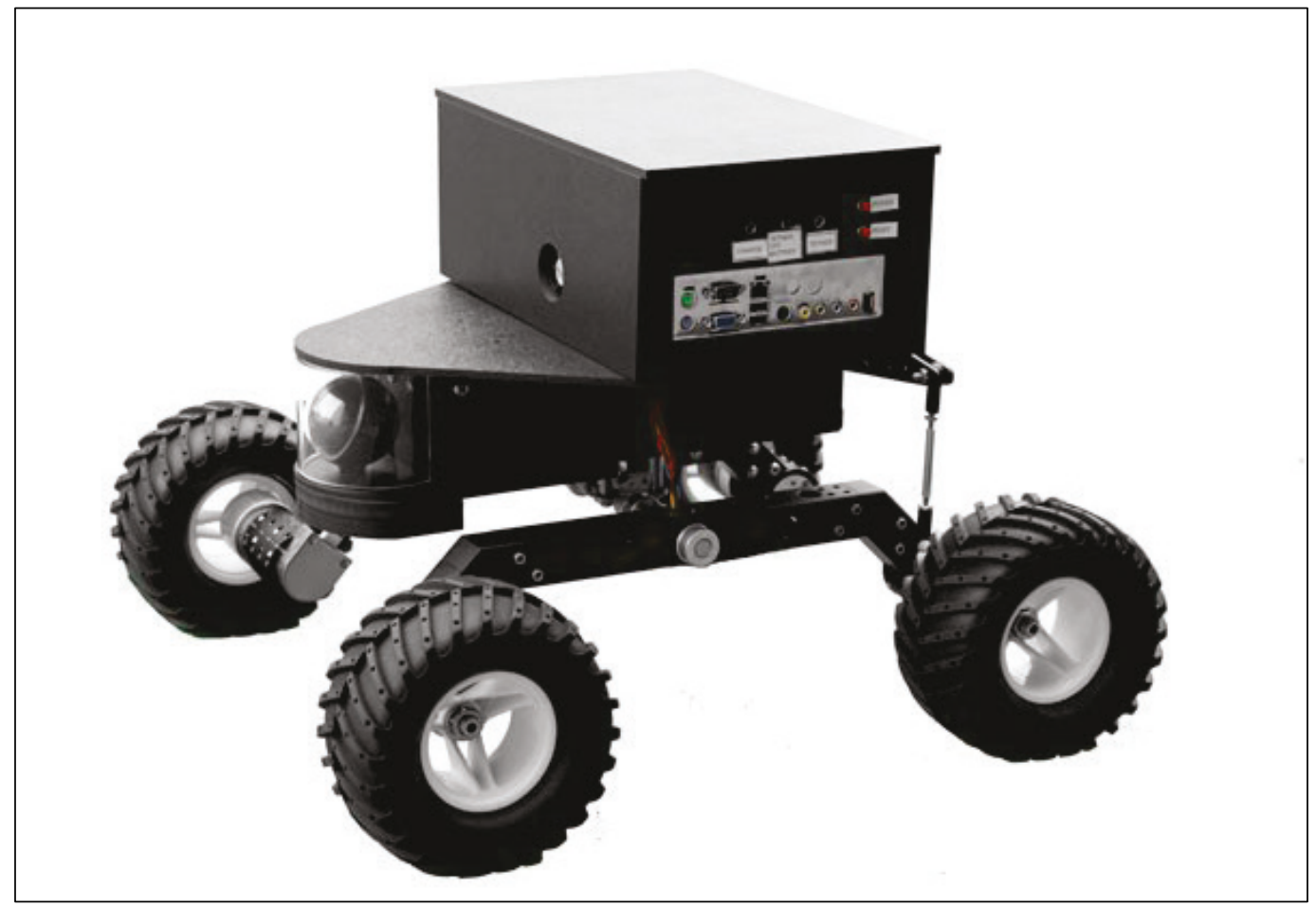

Figure 8. Adept Pioneer 3-AT.

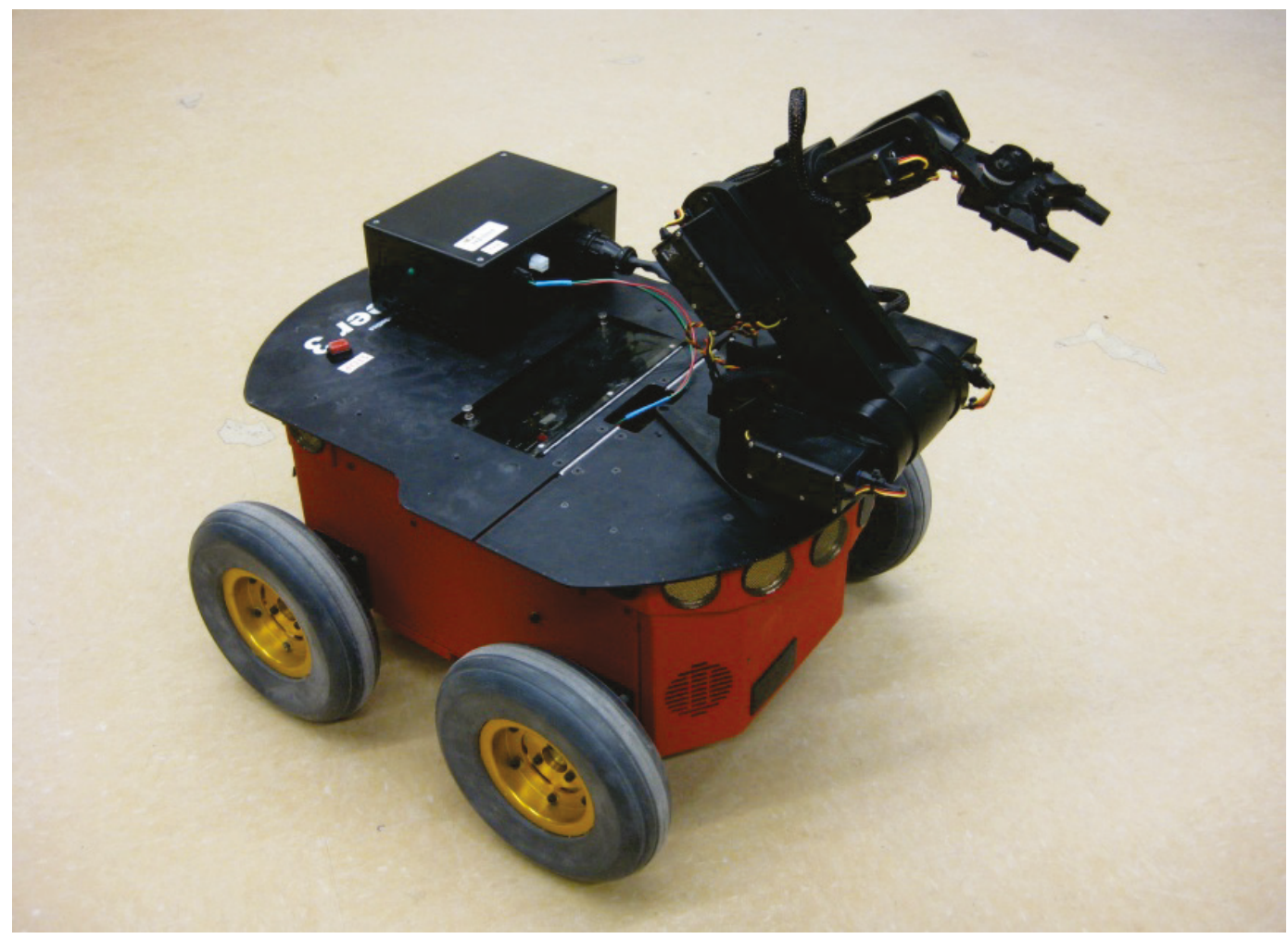


For this project, we selected the Coroware Explorer (Figure 7). The Explorer meets all the above requirements. It has 6-in. clearance or more. It is equipped with two sensors: a two Mega Pixel Color Webcam to capture scenes and a Laser Range Finder (Figure 9) for detection of landmarks. It has a $2.0 \mathrm{GHz} \mathrm{CPU}, 1 \mathrm{~GB}$ of RAM, a $2.0 \mathrm{GHz} \mathrm{CPU}$, a 4-hr battery life, a dual-boot operating system (Ubuntu Linux or Windows), Wi-Fi, and a CUDA-capable main board. Its dimensions are 23 in. long, $21 \mathrm{in}$. wide, and $16 \mathrm{in}$. high with a weight of about $20 \mathrm{lb}$. The robotic software platform is the Robotic Operating System. Appendix A contains an original quote for the Coroware Explorer from Coroware.

Figure 9. Hokuyo laser range finder.

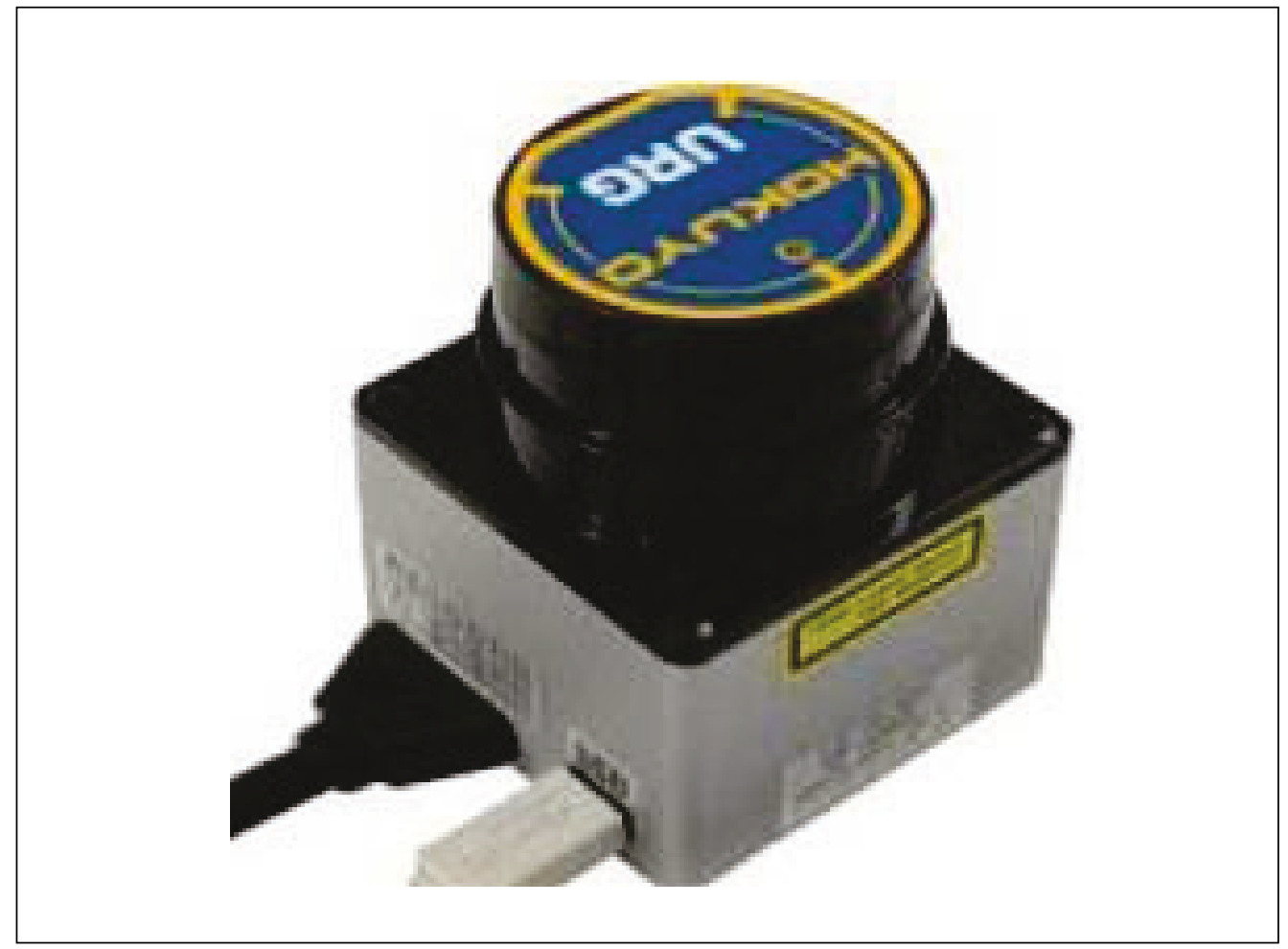

The Laser Range Finder, shown in Figure 9, is the Hokuyo UTM-30L-X. The Hokuyo UTM-30L-X has a detectable range of $100 \mathrm{~mm}$ to $30,000 \mathrm{~mm}, 25 \mathrm{msec}$ per scan, $12 \mathrm{~V}$ operating voltage, and a $270 \mathrm{deg}$ scanning range. It connects to the Explorer via USB. The cost of the Hokuyo UTM-30L-X was approximately $\$ 7,000$.

\subsection{Description of software}

The Robotic Operating System (ROS), an Open Source UGV software, was selected for use with this project. ROS provides libraries and tools to help 
software developers create robot applications. ROS is not an operating system in the traditional sense of process management and scheduling; rather it provides a structured communication layer above the host operating system of a heterogeneous compute cluster (Quigley et al. 2009).

ROS was designed to meet a specific set of challenges encountered when developing large-scale service robots as part of the STAIR project at Stanford University and the Personal Robots Program at Willow Garage, but the resulting architecture is far more general than service-robot and mobile-manipulation domains. The philosophical goals of ROS can be summarized as (1) peer-to-peer, (2) tools-based, (3) multi-lingual, (4) thin, and (5) free and open-source.

ROS provides services expected of an operating system, including hardware abstraction, low-level device control, implementation of commonly used functionality, message-passing between processes, and package management (http://wiki.ros.org/Ros/Introduction). It also provides tools and libraries for obtaining, building, writing, and running code across multiple computers (Quigley et al. 2009).

ROS currently only runs on Unix-based platforms. Software for ROS is primarily tested on Ubuntu and Mac OS X systems, although the ROS community has been contributing support for Fedora, Gentoo, Arch Linux, and other Linux platforms (Quigley et al. 2009).

\subsection{Mapping and localization algorithms}

Thus far, grid-based, topological, and hybrid mapping algorithms were studied for possible use to perform this research. Markov Localization, the Monte Carlo Method, and Kalman filters have been studied as a means to perform localization of the robot. SLAM has been studied and performs localization and mapping simultaneously.

\subsection{Summary}

The Coroware Explorer was selected as the platform for this research. ROS was used to program the Explorer robotic platform. Three mapping and localization algorithms will be implemented and compared in Chapter 4 . 


\section{Simulation Testing Evaluation}

This chapter provides a quantitative evaluation of three laser-based SLAM algorithms implemented in the 2-D simulator: CoreSLAM, Gmapping, and HectorSLAM. The tele-operated robot explores three ground truth map images, and each produces a generated map image. Image registration, the process of aligning two images of the same scene, is then used to align the ground truth map and the generated map for a multimodal comparison of the two. The Hausdorff Distance, a mathematical function used to measure the difference between two subsets of the same space, is then calculated to show the difference in the two images as a numeric value. Both image registration and the Hausdorff distance code are written in MATLAB and produce results comparing each of the algorithms and showing one algorithm is superior to the other two.

\subsection{Evaluated SLAM algorithms}

As discussed in Section 2.4, many types of SLAM algorithms exist, for example, those that are vision-based or laser-based, and those that are 2-D or 3-D. The three algorithms evaluated were available at www.ros.org: CoreSLAM, Gmapping, and HectorSLAM. Each algorithm requires laserbased inputs as data for the simulation. The three SLAM algorithms collect data via the tele-operated robot within the Stage 4.1.1 software simulation, each outputting a map image of the navigated environment.

Each algorithm is used as a black box in this research; however, the underlying details of the implementation differ in the following aspects:

(1) Both HectorSLAM and CoreSLAM rely on scan matching, while Gmapping uses particle filters, (2) CoreSLAM may produce a different map each time with the same input dataset, and (3) CoreSLAM requires loop closing while Hector SLAM does not.

\subsubsection{CoreSLAM}

CoreSLAM is a version of SLAM that implements tinySlam. It requires a mobile robot that provides odometry data and is equipped with a horizontally mounted, fixed, laser range-finder. The slam_CoreSLAM node will attempt to transform each incoming scan into the Odom (odometry) "tf" frame. 
CoreSLAM relies on a simple Monte Carlo algorithm for scan matching and was developed by Steux and El Hamzaoui with the goal of producing a SLAM algorithm with no more the 200 lines of codes. CoreSLAM has aparticle filter routine, ts_distance_scan_to_map, and a map update function. The ts_distance_scan_to_map routine tests each state position, and the map update function updates the map as the robot navigates its environment.

The slam_CoreSLAM node takes as input laser data and pose data collected from the laser range finder and outputs a low quality map, yet a recognizable one.

Overall, CoreSLAM performs better on a slow robot.

\subsubsection{Gmapping}

Gmapping is a highly efficient Rao-Blackwellized particle filter to learn grid maps from laser range data. Implementation requires a mobile robot equipped with a mounted, fixed, laser range finder.

Loop closure is the hardest part; when closing a loop, be sure to drive another 5 to $10 \mathrm{~m}$ to get plenty of overlap between the start and end of the loop.

This package contains Gmapping from OpenSlam and a ROS wrapper. The Gmapping package provides laser-based SLAM as a ROS node called slam_Gmapping. Using slam_Gmapping, it creates a 2-D occupancy grid map (like a building floor plan) from laser range finder and pose data collected by a mobile robot (Santos 2008).

The slam_Gmapping node takes as input laser data and pose data collected from the laser range finder and outputs a high quality map.

\subsubsection{Hector SLAM Gmapping}

Hector SLAM relies on scan matching, uses a Gauss-Newton Approach, and is accurate enough that it does not require loop closure. The Hector SLAM package consists of three main packages, hector_mapping, hector_geotiff, and hector_trajectory_server. 
The Hector_mapping node is a SLAM approach used with or without odometry on platforms that exhibit roll/pitch motion (of the sensor, the platform, or both). It leverages the high update rate of modern LIDAR systems like the Hokuyo UTM-3OLX and provides 2-D pose estimates at the scan rate of the sensors ( $40 \mathrm{~Hz}$ for the UTM-30LX). Although the system does not provide an explicit loop closing ability, it is sufficiently accurate for many real-world scenarios. The system has been used successfully on Unmanned Ground Robots, Unmanned Surface Vehicles, Handheld Mapping Devices, and logged data from quadrotor UAVs (Kohlbrecher 2011).

Hector_geotiff saves the map and robot trajectory to geotiff image files. The hector_trajectory_server saves trajectory files as output. The hector_mapping node's main input is scan data on the /scan topic. The data are then transformed via the /tf topic.

Overall, Hector SLAM outputs a high quality map that is recognizable.

\subsection{Ground truth maps}

Figure 10 displays the three maps chosen as the ground truth maps for the simulation, labeled as Map One, Map Two, and Map Three. Each is simple in design and simple to navigate. All three maps were downloaded from the web and used with the Stage 4.1.1 simulator as the ground truth map for the simulated robot to navigate.

Figure 10. Ground truth maps.

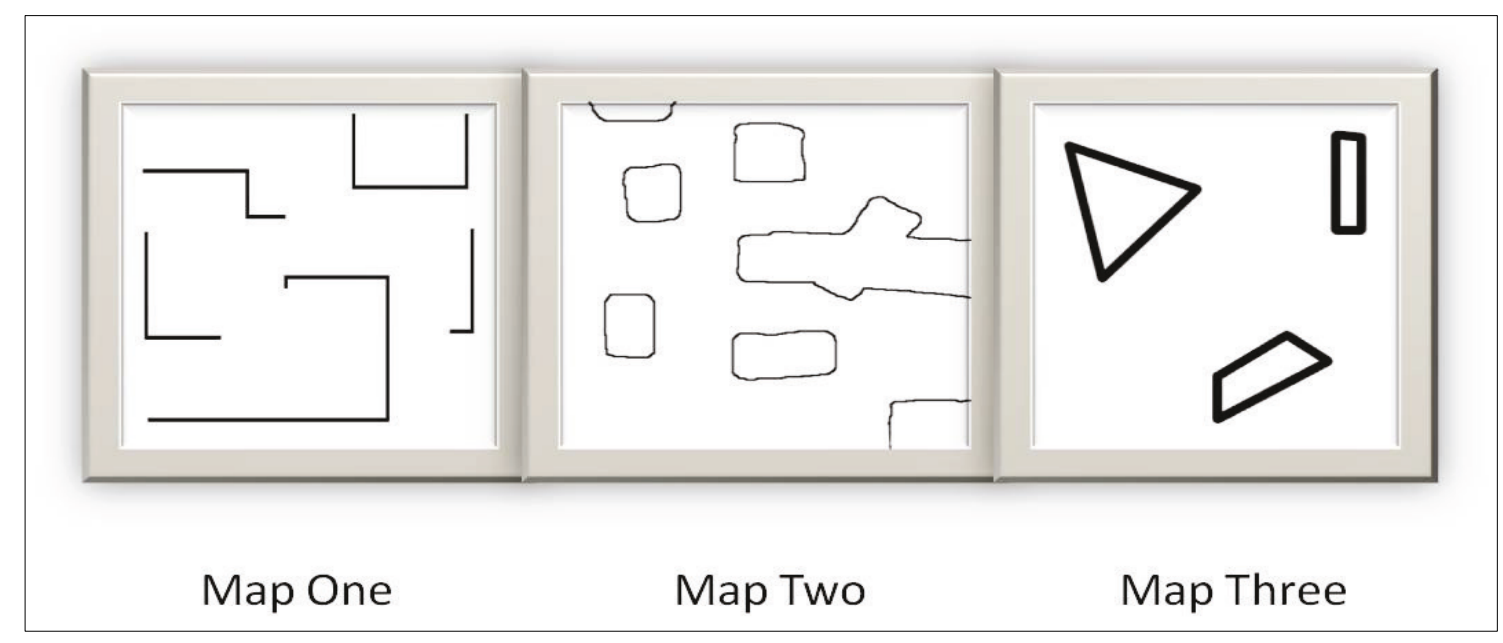




\subsection{Simulation results}

Each of the three SLAM algorithms previously discussed was tested using the 2-D simulation environment, Stage, a 2-D robotic simulator that provides users with the capabilities of simulating a robot or a variety of robots in an environment or a variety of environments. Stage, an opensource software, provides multiple physics-based models for robot sensors and actuators. Some of the currently supported models are sonar and infrared rangers, 2-D scanning laser rangefinder, color-blob tracking, fiducial tracking, bumpers, grippers, and mobile robot bases with odometric and global localization (University of Tennessee Knoxville 2007).

One advantage Player/Stage provides is the ability to move from simulation to the robot by changing a few parameters (Staranowicz and Mariottini 2011). The learning curve on the Stage software is a disadvantage.

Stage, used standalone or with ROS, has many versions. This research implements Stage 4.1.1, the most recent version and requires ROS Fuerte for implementation.

The simulated robot completely navigated each of the three ground truth maps with each of the three algorithms to produce the three generated maps. Figure 11 shows the ground truth map, labeled Fixed, and the three generated maps produced by each algorithm, labeled CoreSLAM, Gmapping, and Hector Slam, after navigating the ground truth of Map One. Figure 12 shows the ground truth map, labeled Fixed, and the three generated maps produced by each algorithm, labeled CoreSLAM, Gmapping, and Hector Slam, after navigating the ground truth of Map Two. Figure 13 shows the ground truth map, labeled Fixed, and the three generated maps produced by each algorithm, labeled CoreSLAM, Gmapping, and Hector Slam, after navigating the ground truth of Map Three.

Analyses of these maps are discussed later. 
Figure 11. Map One and generated map of each algorithm.

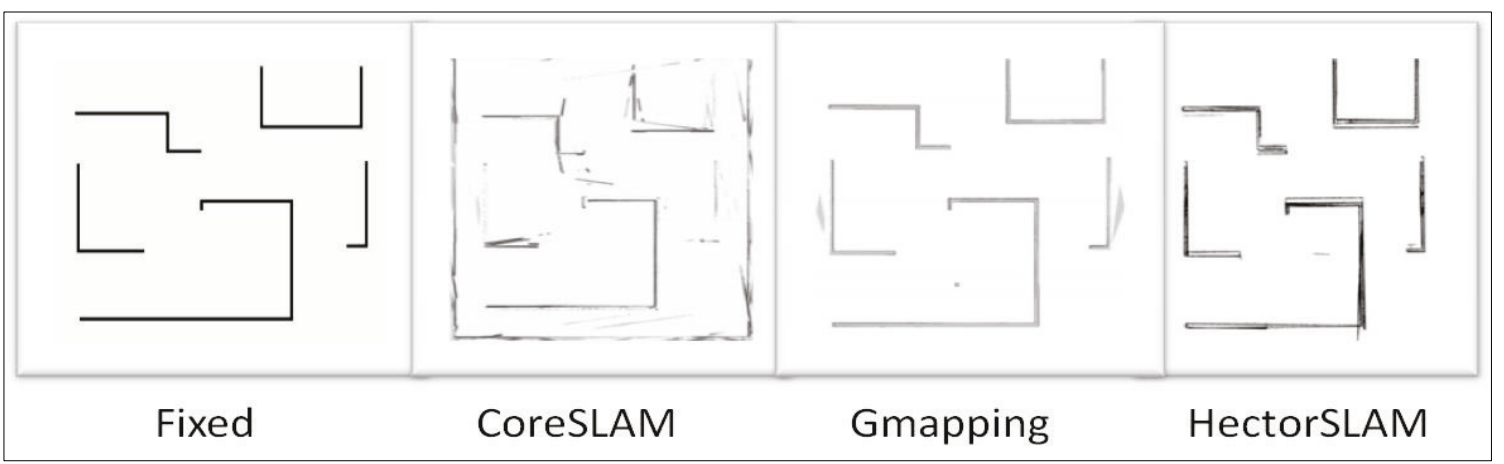

Figure 12. Map Two and generated map of each algorithm.

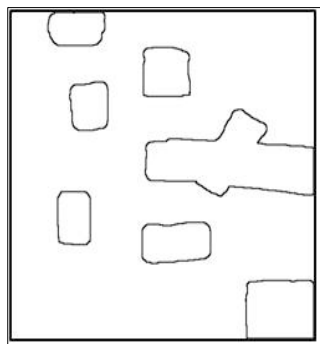

Fixed

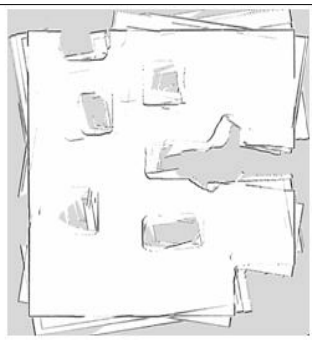

CoreSLAM

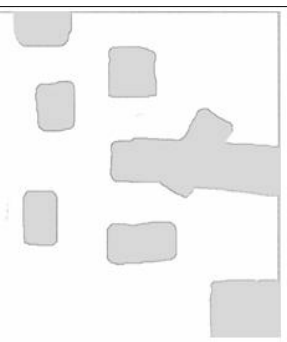

Gmapping

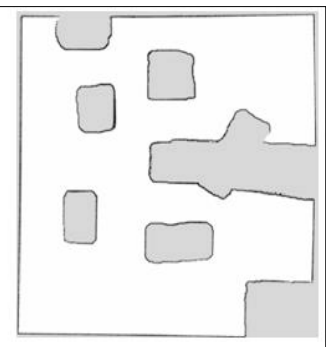

HectorSLAM

Figure 13. Map Three and generated map of each algorithm.

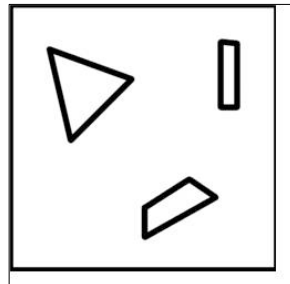

Fixed

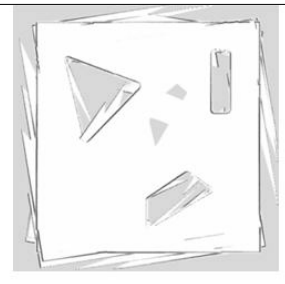

CoreSLAM

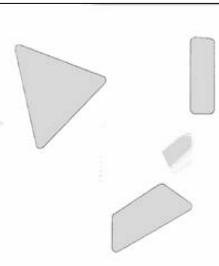

Gmapping

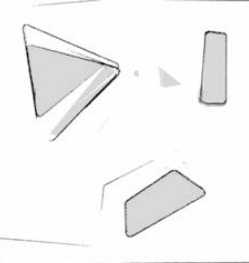

HectorSLAM

\subsection{Image registration of ground truth and generated maps}

MATLAB (matrix laboratory) is a multi-paradigm numerical computing environment and fourth-generation programming language with many functions and libraries. This research uses the image registration tool to compare the generated maps to the ground truth map.

Image registration, the process of aligning two images of the same scene, is then used to align the ground truth map image and the generated map 
image for a multimodal comparison of the two images. An intensity-based automatic image registration process requires a pair of images, a metric, an optimizer, and a transformation type in order to align one image with another. The pair of images is the ground truth map image and the generated map image.

The metric defines the image similarity metric for evaluating the accuracy of the registration. The optimizer defines the methodology for minimizing or maximizing the similarity metric.

The transformation type defines the type of 2-D transformation that brings the misaligned image (called the moving image or the generated image) into alignment with the reference image (called the fixed image or the ground truth image). Four transform types exist: affine, rigid, similar, and translation.

The image registration process begins with the transform type you specify and an internally determined transformation matrix. Together, they determine the specific image transformation that is applied to the moving image with bilinear interpolation.

Next, the metric compares the transformed moving image to the fixed image and a metric value is computed.

Finally, the optimizer checks for a stop condition. A stop condition is anything that warrants the termination of the process. In most cases, the process has reached a point of diminishing returns or it has reached the specified maximum number of iterations. If there is no stop condition, the optimizer adjusts the transformation matrix to begin the next iteration.

The following sections discuss the resulting aligned images produced with the four transformations, i.e., affine, rigid, similar, and translation, and the three algorithms. The maps will contain three colors: magenta, green, and black. The magenta represents the intensity of the ground truth image, the green represents the intensity of the SLAM generated map, and the black represents where both images align or are the same.

\subsubsection{CoreSLAM}

Figures 14, 15, and 16 were produced using two images, (1) the ground truth map images, and (2) the Stage simulated generated map images 
from the CoreSLAM algorithm, as inputs to MATLAB's image registration function. In general, image registration overlays or aligns the generated map on to the ground truth map image to compare the two images. The image registration tool is executed with the four different transform types: affine, translation, rigid, and similar. Figures 14, 15, and 16 display the output images of the four transformations with the MATLAB code.

The generated maps show up with more intensity than the ground truth maps due to CoreSLAM producing multiple edges (green shaded areas) along the exterior portion of the map. While the ground truth and generated maps are similar in nature, there are few overlapping points because there is very little black, which shows points where the two images are identical.

Figure 14. Image registration of Map One and CoreSLAM generated map.

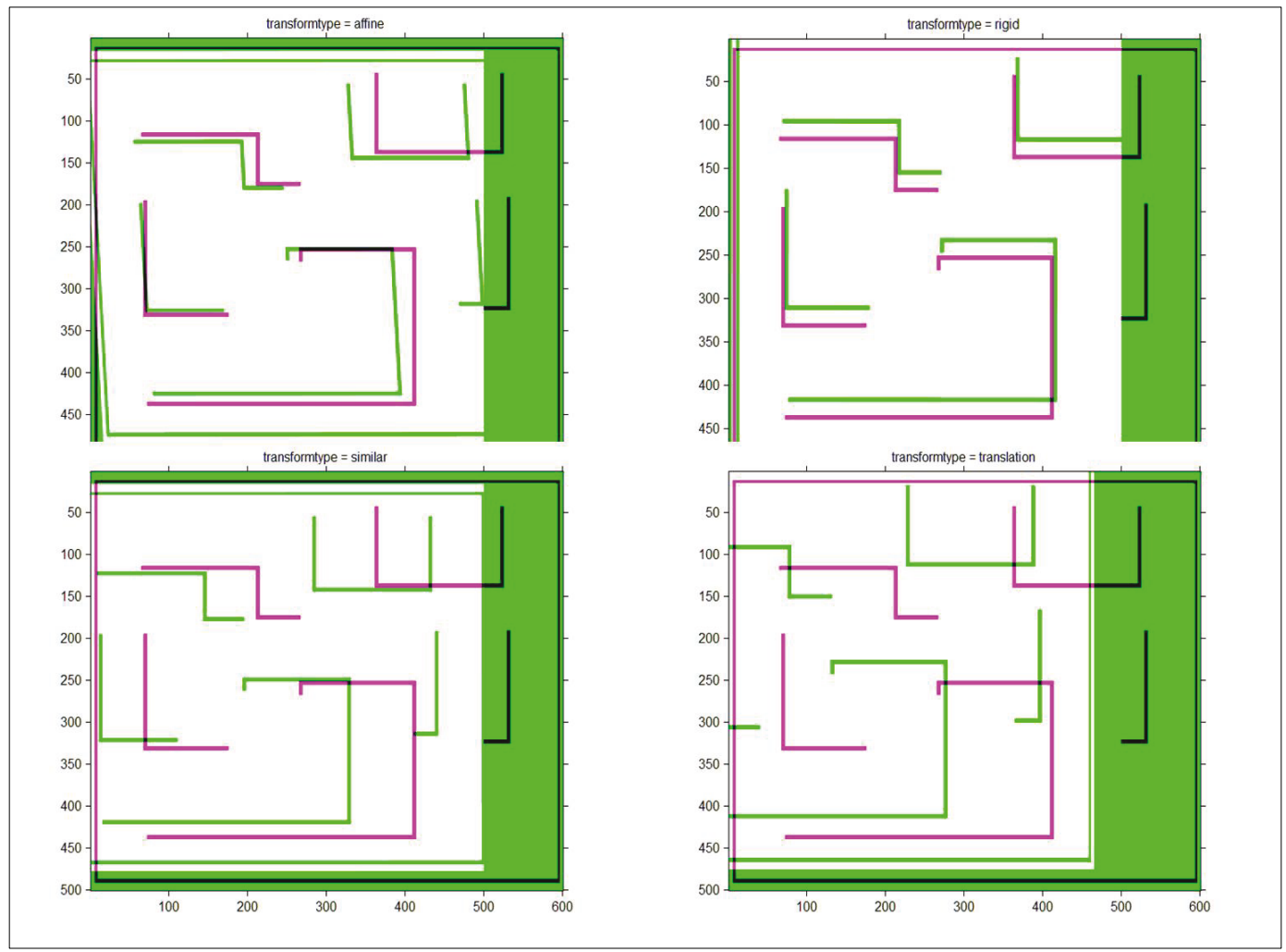


Figure 15. Image registration of Map Two and CoreSLAM generated map.

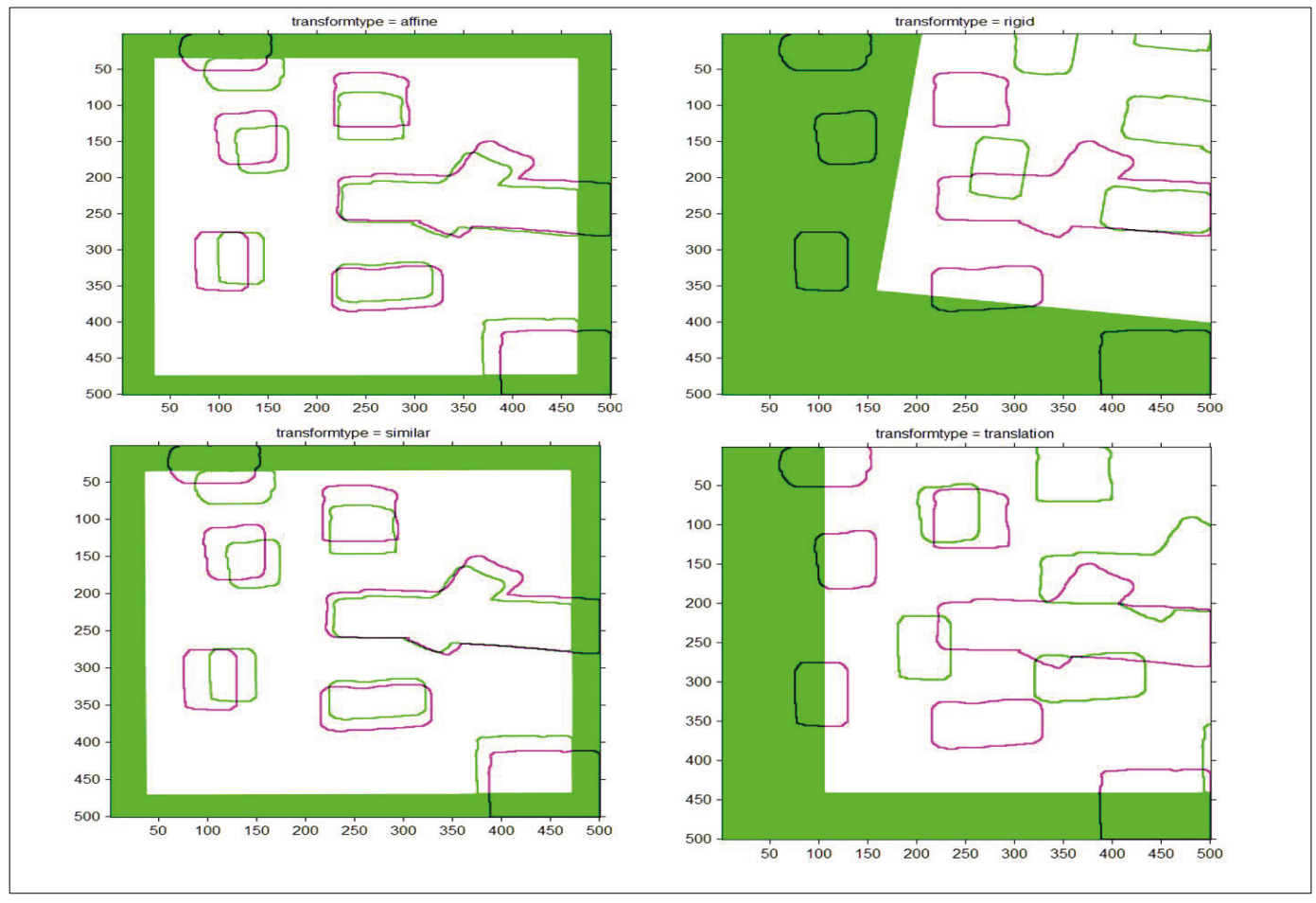

Figure 16. Image registration of Map Three and CoreSLAM generated map.

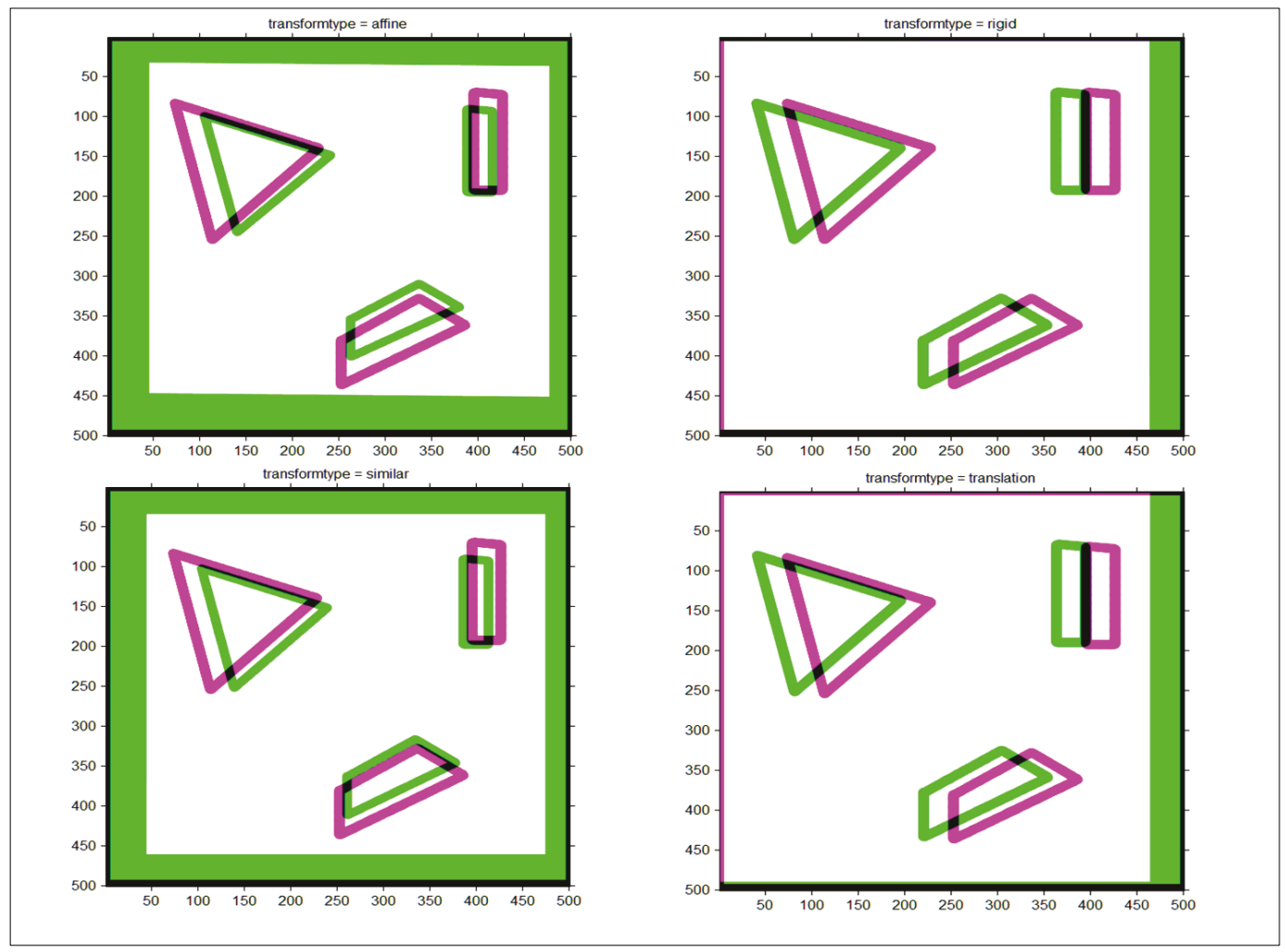




\subsubsection{Gmapping}

Figures 17,18 , and 19 were produced using two images; the ground truth map images and the Stage simulated generated map images from the Gmapping algorithm in MATLAB's image registration function. The magenta in the image of Figure 17 represents the intensity of the ground truth image, the green represents the intensity of the SLAM generated map, and the black represents where the images overlap. In the upper left of Figure 18, all transform types align all most perfectly. Transform type affine has a small amount of green in the bottom indicating that the ground truth map of Map Two has a slightly higher intensity than the generated map. Transform type translation (bottom right) has a small amount of green on the left and the bottom of the map, indicating the ground truth map of Map Two has a slightly higher intensity than the generated map. In Figure 19, the magenta is stronger in all transformations, indicating that the generated map has a higher intensity than the ground truth of Map Three. The black shows where the images align.

Figure 17. Image registration of Map One and Gmapping generated map.

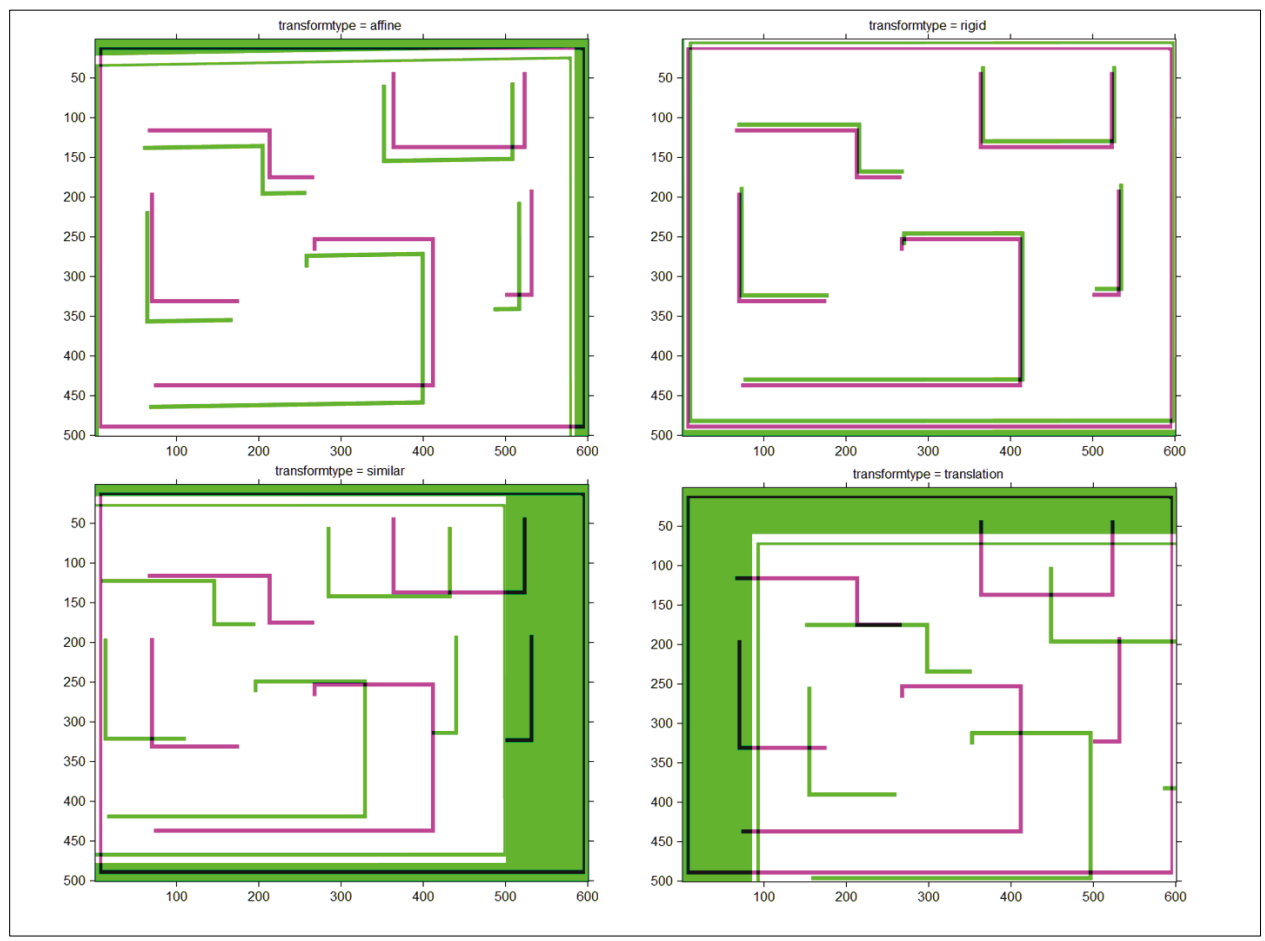


Figure 18. Image registration of Map Two and Gmapping generated map.

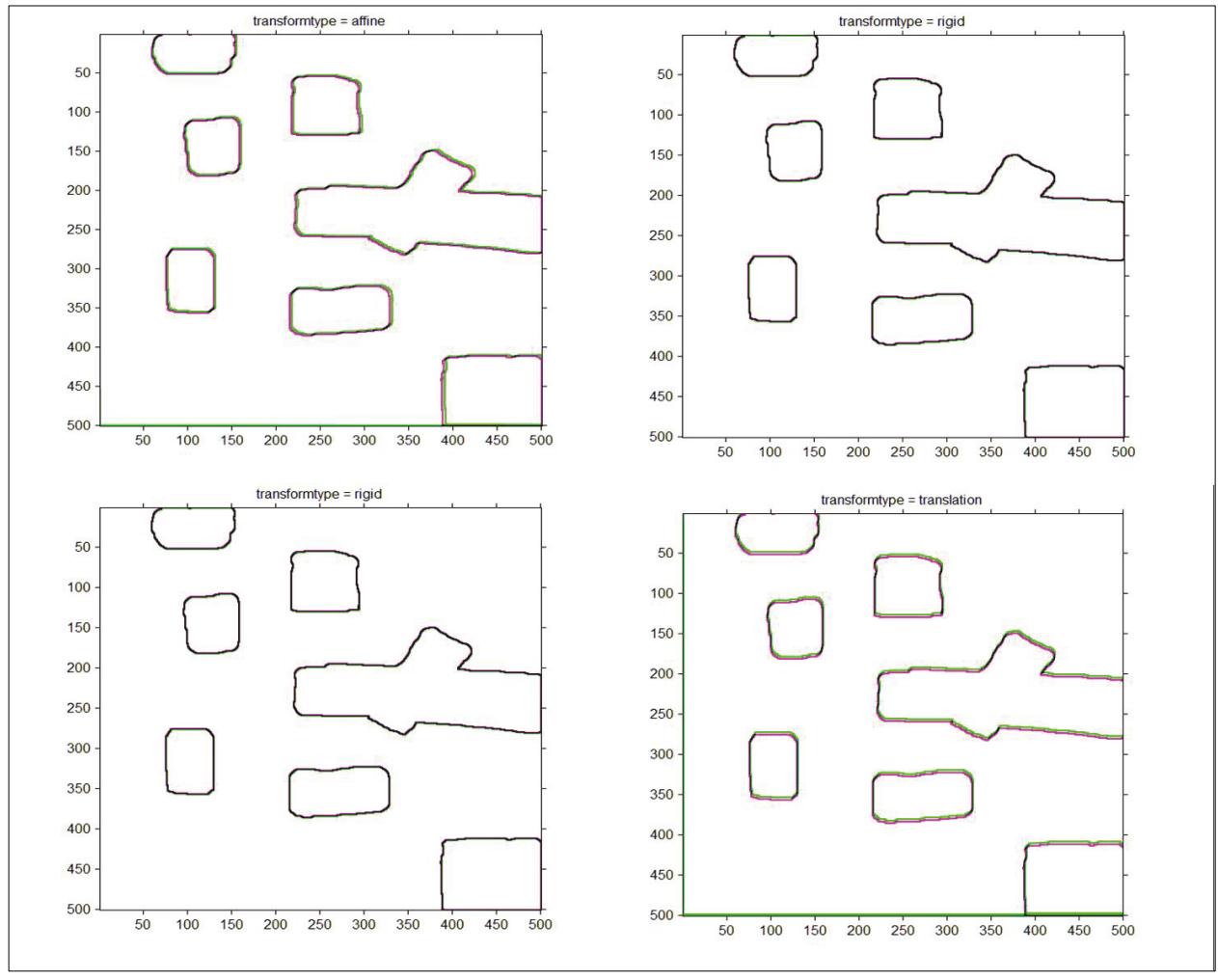

Figure 19. Image registration of Map Three and Gmapping generated map.

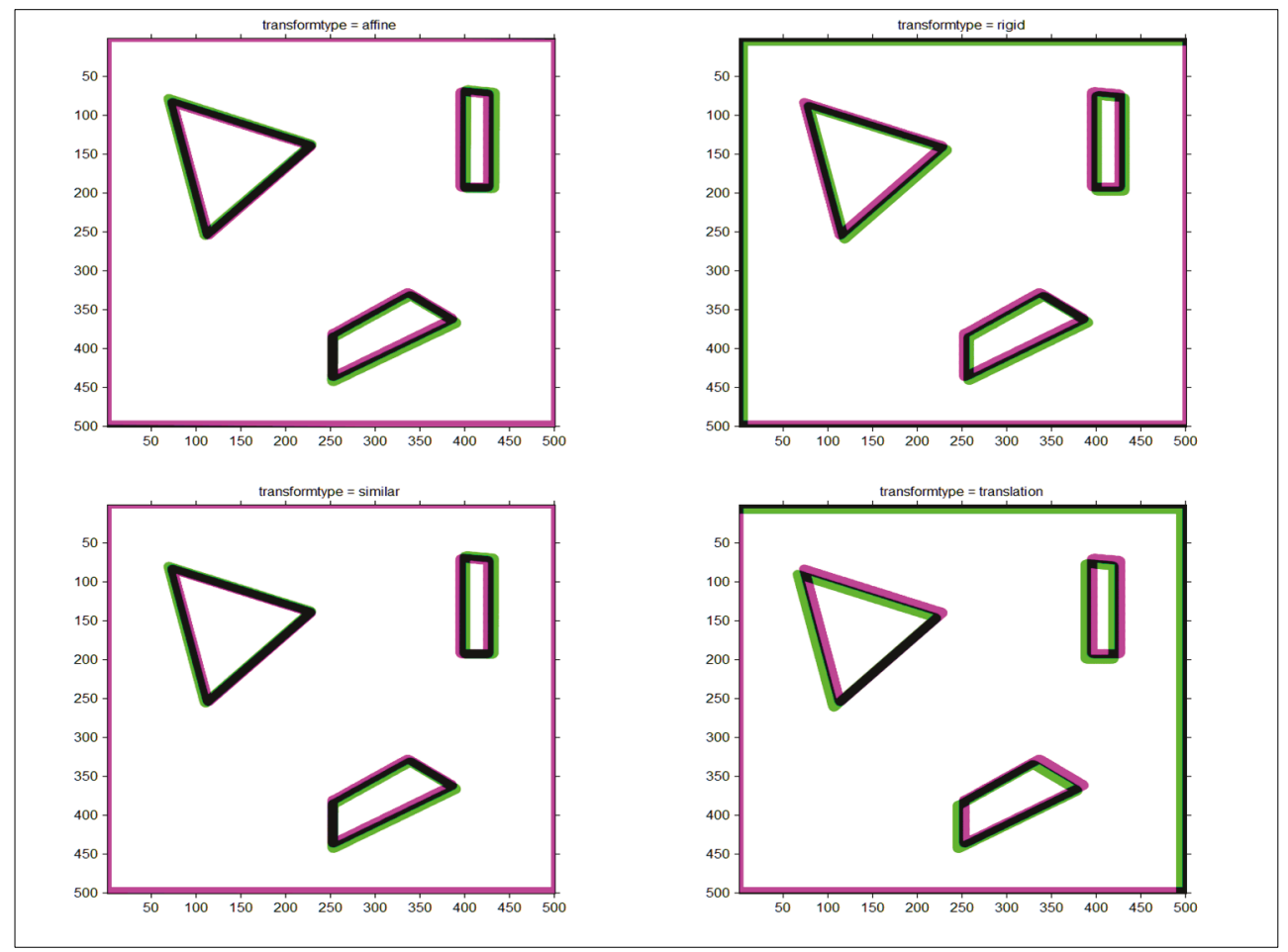




\subsubsection{Hector SLAM}

Figures 20, 21, and 22 were produced by using the ground truth maps and the Stage simulated generated maps with the Hector SLAM algorithm as input in MATLAB's image registration function. The image registration tool was again processed with the four different transform types: affine, translation, rigid, and similar. Figure 20 shows the generated map has more intensity than the ground truth map due to the alignment being off and the degree of difference in the two maps. While the maps are similar in nature, they have few overlapping points. Figures 21 and 22 show black more than magenta and green indicating that the two images have very little differences. Figure 20 has more green with all transform types.

Figure 20. Image registration of Map One and Hector SLAM generated map.

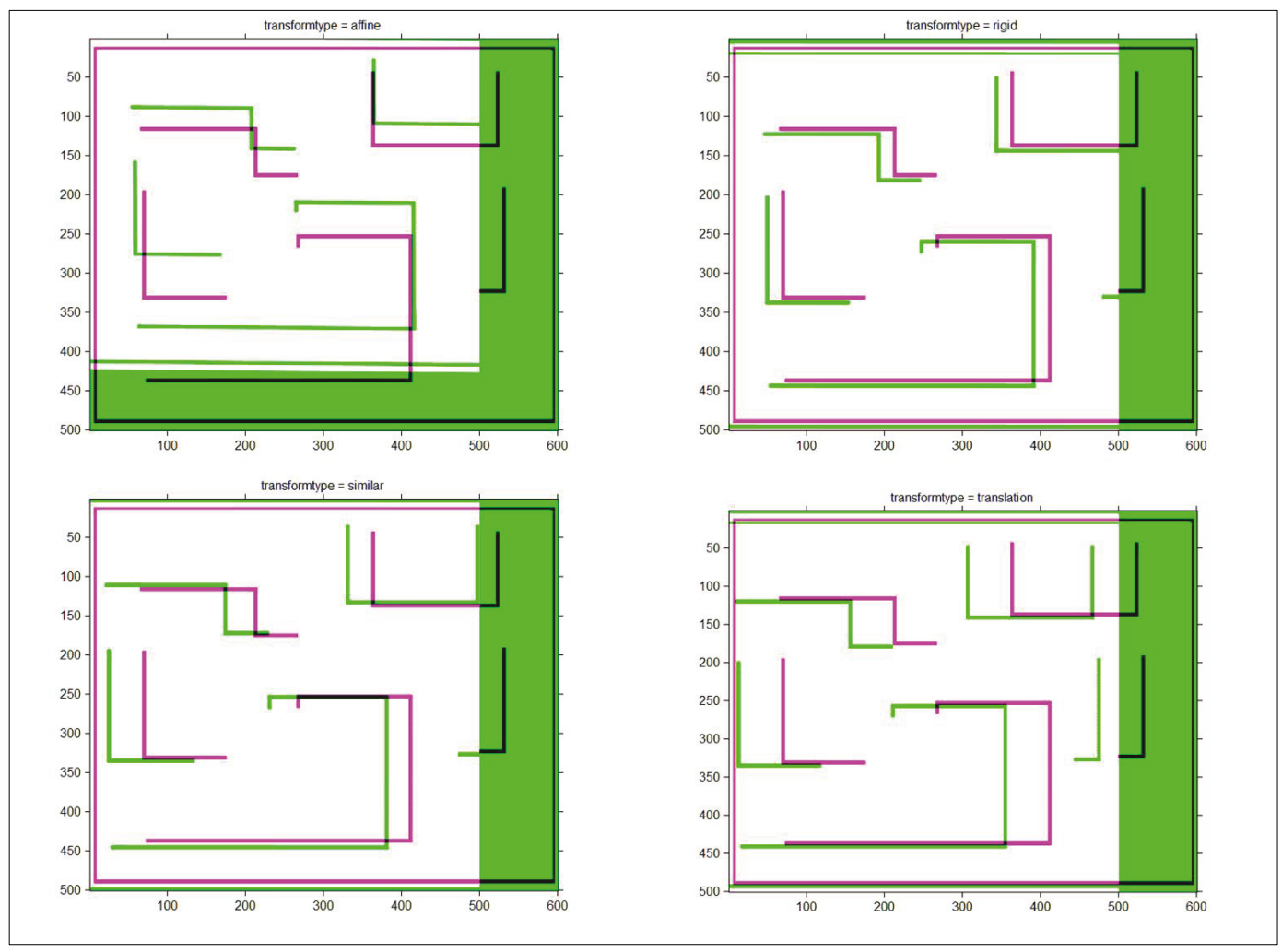


Figure 21. Image registration Map Two and Hector SLAM generated map.

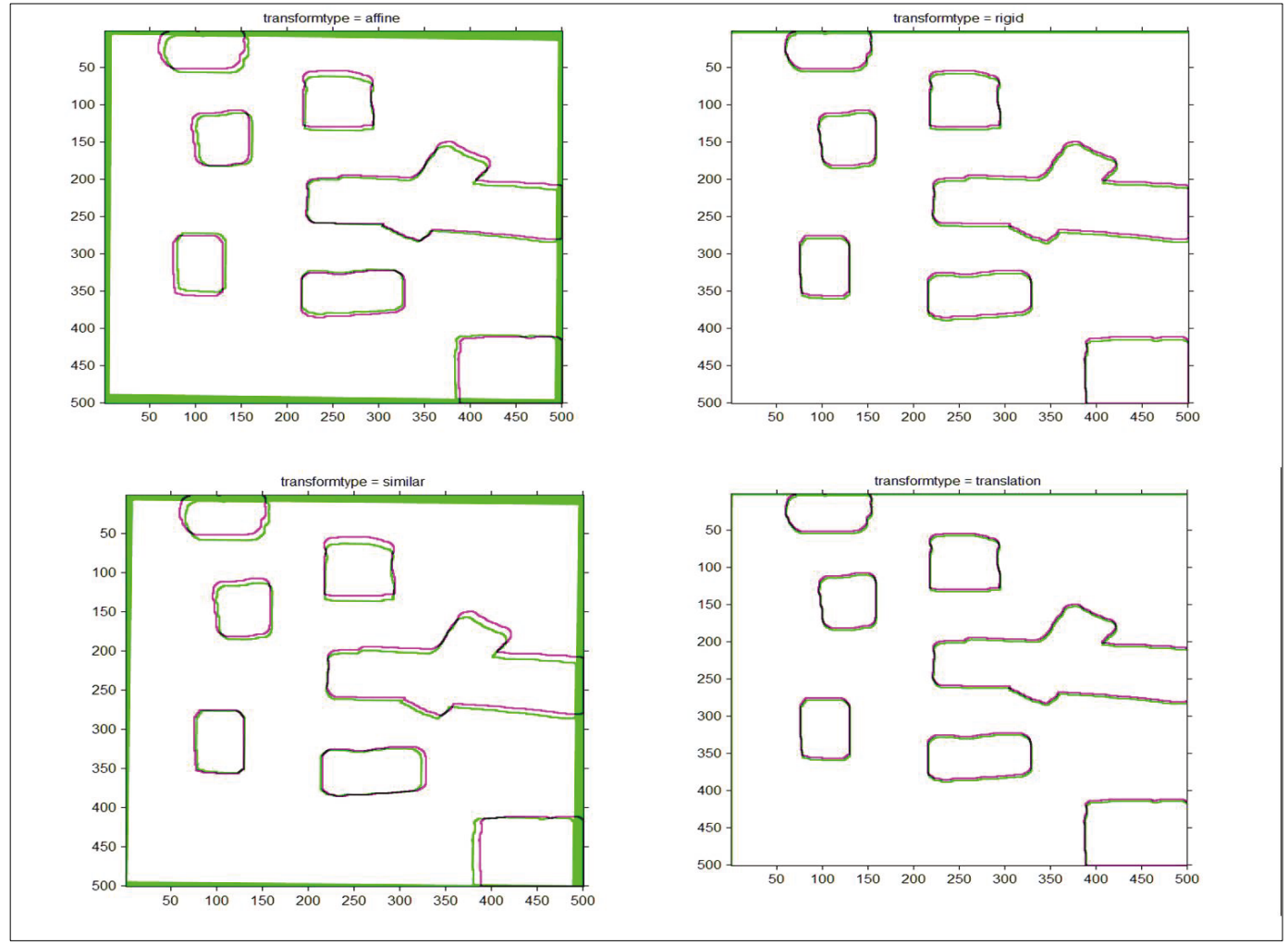

Figure 22. Image registration of Map Three and Hector SLAM generated map.

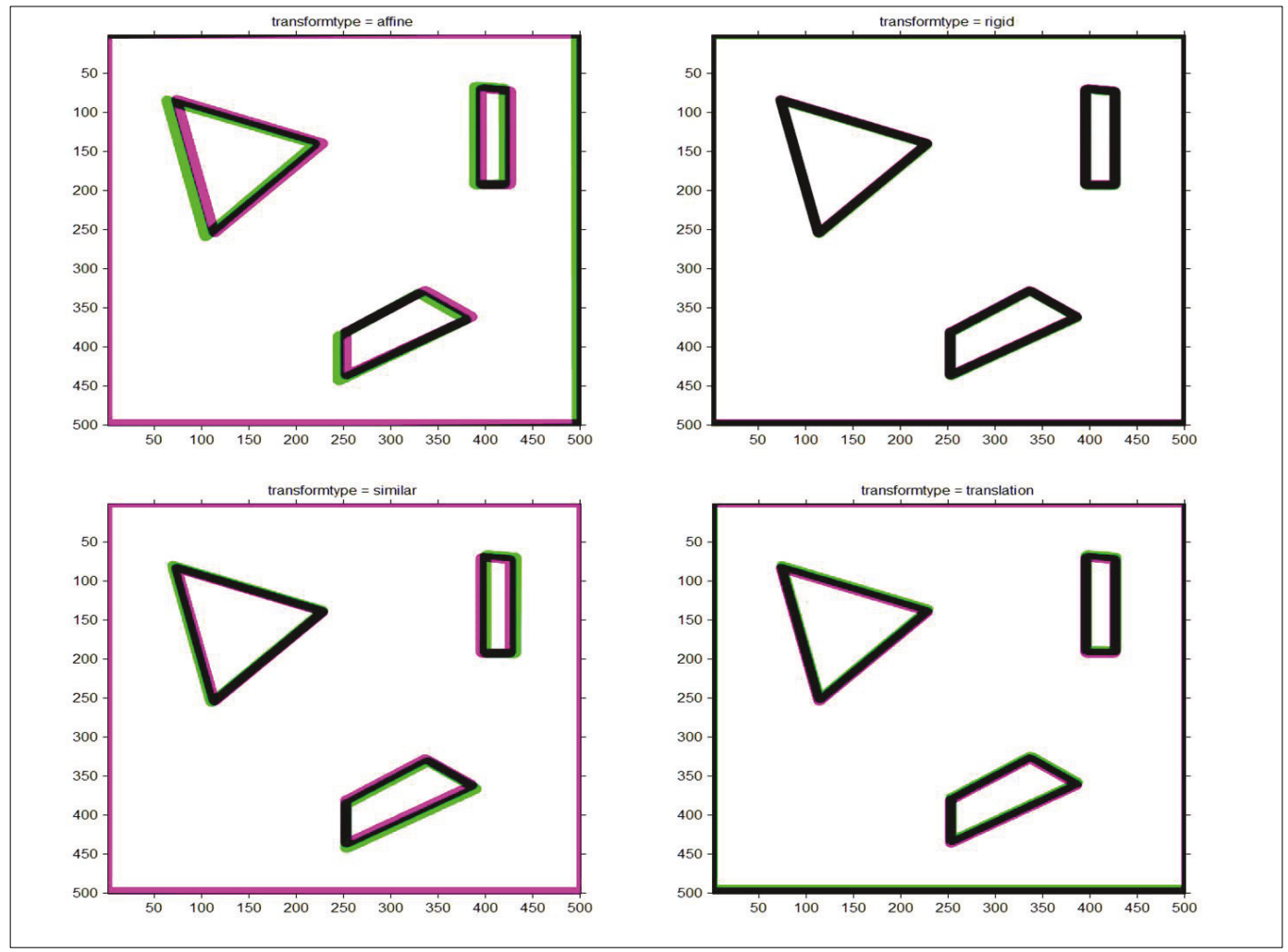


Figure 21 shows green around the exterior due to the processing of image. In Figures 21 and 22, the rigid (upper right) and translation (lower right) transforms produce near perfect alignments.

\subsubsection{Comparisons of ground truth maps and Stage 4.1.1 generated maps}

For a quantitative measure between the ground truth maps and the generated simulator maps, the Hausdorff Distance is calculated. The Hausdorff distance, by definition, is as follows: Given two finite sets $A=$ $\left(\mathrm{a}_{1} \ldots . . \mathrm{a}_{\mathrm{p}}\right)$ and $B=\left(\mathrm{b}_{1} \ldots \mathrm{b}_{\mathrm{p}}\right)$, the distance is calculated as

$$
H(A, B)=\max (h(A, B), h(B, A))
$$

where

$$
h(A, B)=\underset{a \in A b \in B}{\sup \inf \|a-b\|}
$$

|| $\quad$ || represents some underlying norm defined in the space of the two point sets, which is generally required to be an $\mathrm{L}_{\mathrm{p}}$ norm, usually the $\mathrm{L}_{2}$ or Euclidean norm. The function $h(A, B)$ is called the directed Hausdorff distance from $A$ to $B$. If $A$ and $B$ are compact sets, then

$$
h(A, B)=\max _{a \in A} \min _{b \in B}\|a-b\|
$$

The Hausdorff Distance is calculated with the function $h(A, B)$, which returns the distance of matrix $A$ from matrix $B$. It identifies the point an element of $A$ that is the farthest from any point in $B$ and measures the distance from $A$ to its nearest neighbor in $B$ (comparing images using Hausdorff Distance paper).

Table 1 shows the HectorSLAM algorithm has the lower values of 15.5885 , in the Hausdorff Distance Column. CoreSLAM has the second lowest value of 16.55. Gmapping has the highest value of 22.7156.

Table 2 shows the CoreSLAM algorithm has the lower value of 15.5563, in the Hausdorff Distance Column. HectorSLAM and Gmapping have an equal value of 16.8523 . 
Table 3 shows the HectorSLAM algorithm has the lower values of 14.1067, in the Hausdorff Distance Column. CoreSLAM having the second lowest value of 18.13857 . Gmapping has the highest value of 19.2354 .

Based on all the results, HectorSLAM outperforms both CoreSLAM and Gmapping for Map One and Map Three. CoreSLAM outperforms HectorSLAM and Gmapping for Map Two while HectorSLAM and Gmapping are tied for Map Two.

Table 1. Hausdorff distance for Map One.

\begin{tabular}{|l|l|l|}
\hline & Map Size & Hausdorff Distance \\
\hline CoreSLAM & $600 \times 500$ & 16.55 \\
\hline Gmapping & $600 \times 500$ & 22.7156 \\
\hline Hector SLAM & $600 \times 500$ & 15.5885 \\
\hline
\end{tabular}

Table 2. Hausdorff distance for Map Two.

\begin{tabular}{|l|l|l|}
\hline & Map Size & Hausdorff Distance \\
\hline CoreSLAM & $500 \times 500$ & 15.5563 \\
\hline Gmapping & $500 \times 500$ & 16.8523 \\
\hline Hector SLAM & $500 \times 500$ & 16.8523 \\
\hline
\end{tabular}

Table 3. Hausdorff distance for Map Three.

\begin{tabular}{|l|l|l|}
\hline Hector SLAM & Map Size & Hausdorff Distance \\
\hline CoreSLAM & $500 \times 500$ & 18.13847 \\
\hline Gmapping & $500 \times 500$ & 19.2354 \\
\hline Hector SLAM & $500 \times 500$ & 14.1067 \\
\hline
\end{tabular}




\section{Physical Testing Evaluation}

This chapter provides the evaluation of three laser-based SLAM algorithms, HectorSLAM, Gmapping, and CoreSLAM, implemented on the mobile robot, the Coroware Explorer. The tele-operated robot explored two physical test areas (ground truth maps) and generated a map of each area with each algorithm. Comparisons of the ground truth maps and the generated maps are discussed below.

\subsection{Evaluated algorithms}

The algorithms used for physical testing are the same as those discussed in Section 4.1. The ultimate goal is to have the Explorer navigate each of the test areas and build a map of the unknown environment with each of the three algorithms. Each algorithm takes scan data from the Laser Range Finder attached to the Explorer and outputs a generated map.

\subsection{Test areas}

Two areas were selected for ground truth maps. Figure 23 is the ground truth map of the foyer, break room, and mail room of Building 3296 at the ERDC, and Figure 24 is the ground truth map of the basement area of Building 3296 used to perform the physical test. The tele-operated Explorer navigated each area, and a map was generated simultaneously using the input from the Laser Range Finder and the three algorithms. The robot was networked with a laptop running UBUNTU and ROS Fuerte with each of the three algorithms. Each area was traversed with one of the selected algorithms to produce the three SLAM maps. The rooms in the basement were empty while the foyer, break room, and mail room had tables, chairs, file cabinets, a sink base, soft drink machines, snack machines, garbage cans, ice machine, and recycle bins. The foyer's entry (Figure 23) is made of glass and has a larger area with two glass doors and an entry leading to the hallway with entry to the breakroom and the mailroom. The basement (Figure 24) consists of a hallway with four rooms and another hallway. Rooms 1, 3, and 4 have one door, while Room 2 has two doors. All four rooms were empty. The hallway was about $5 \mathrm{ft}$ wide and $50 \mathrm{ft}$ in length. 
Figure 23. Ground truth map of foyer and break room.

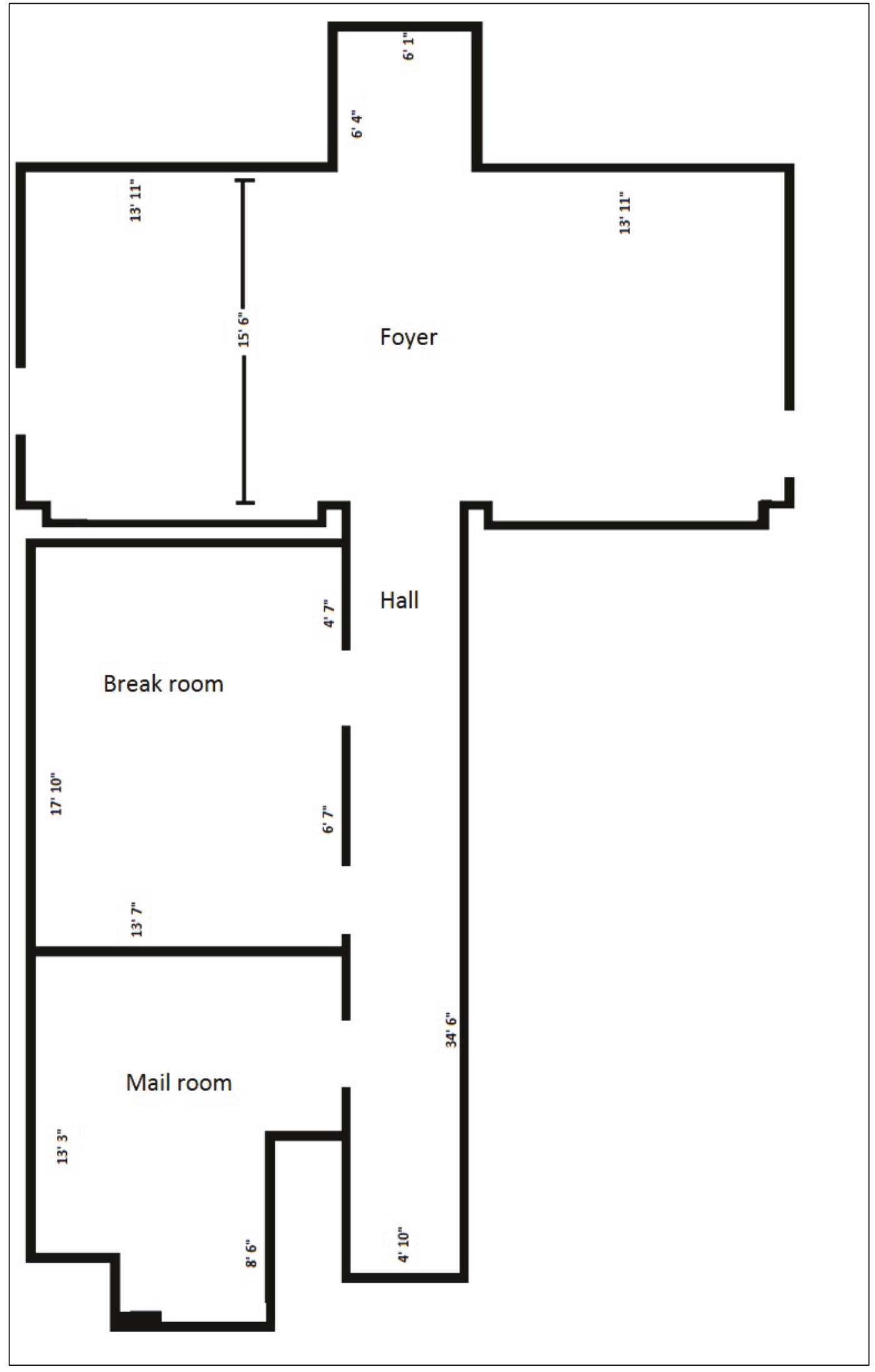


Figure 24. Ground truth map of basement.

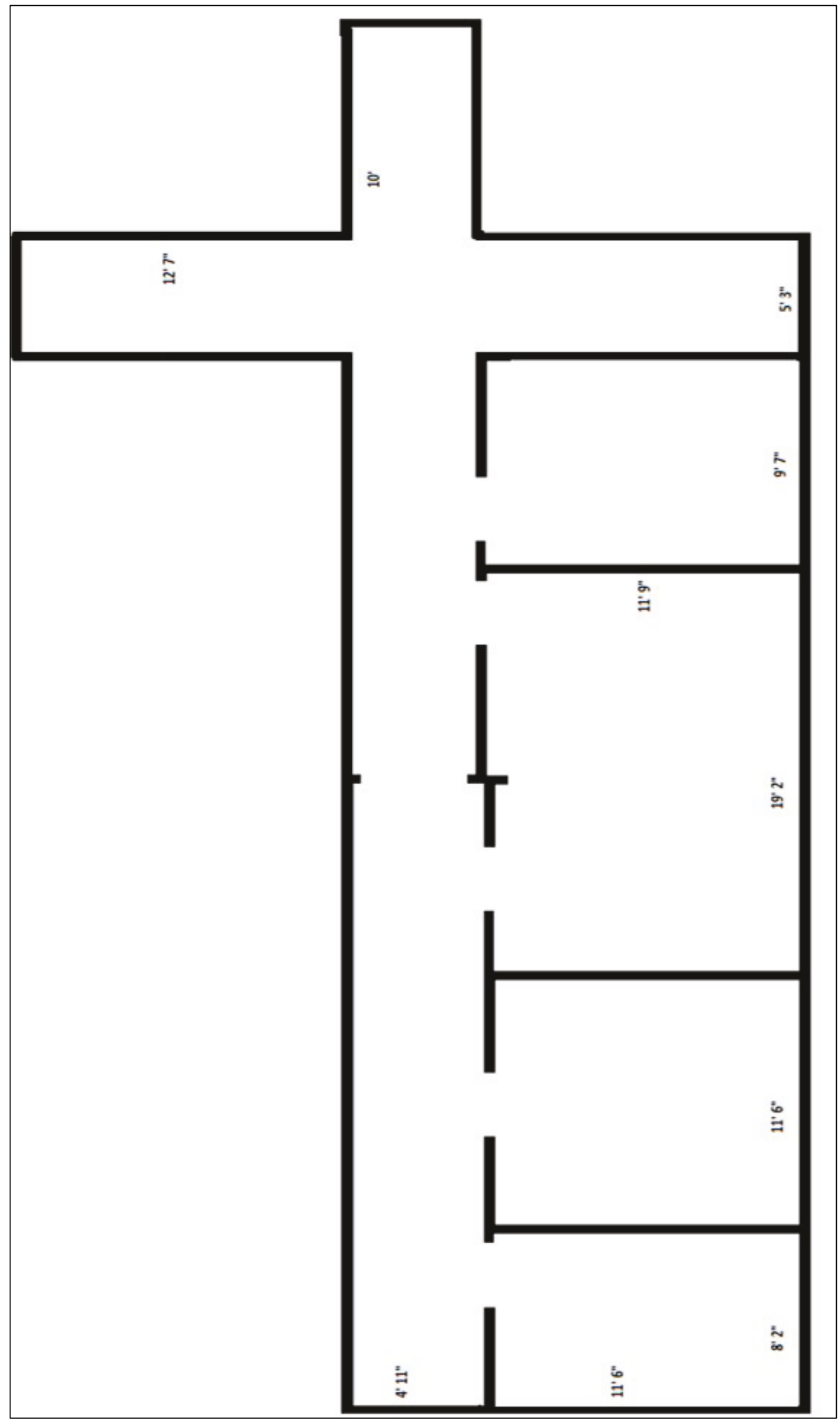




\subsection{Generated maps from physical testing}

This section shows the generated maps produced while navigating the two test areas with the mobile robot. CoreSLAM and Gmapping performed poorly while HectorSLAM generated a recognizable map with many identifiable features.

\subsubsection{CoresLAM}

Figure 25 depicts the generated map from the CoreSLAM for the basement, and Figure 26 depicts the foyer. CoreSLAM performed poorly on the physical robot and the produced maps were of poor quality and unrecognizable compared to the ground truth maps. This could be due to the fact that the four rooms in the basement were small and the robot had to make an almost 360-deg turn in the rooms. However, the map for the foyer was also unrecognizable; therefore, this theory may be incorrect.

Figure 25. Generated CoreSLAM map of basement.

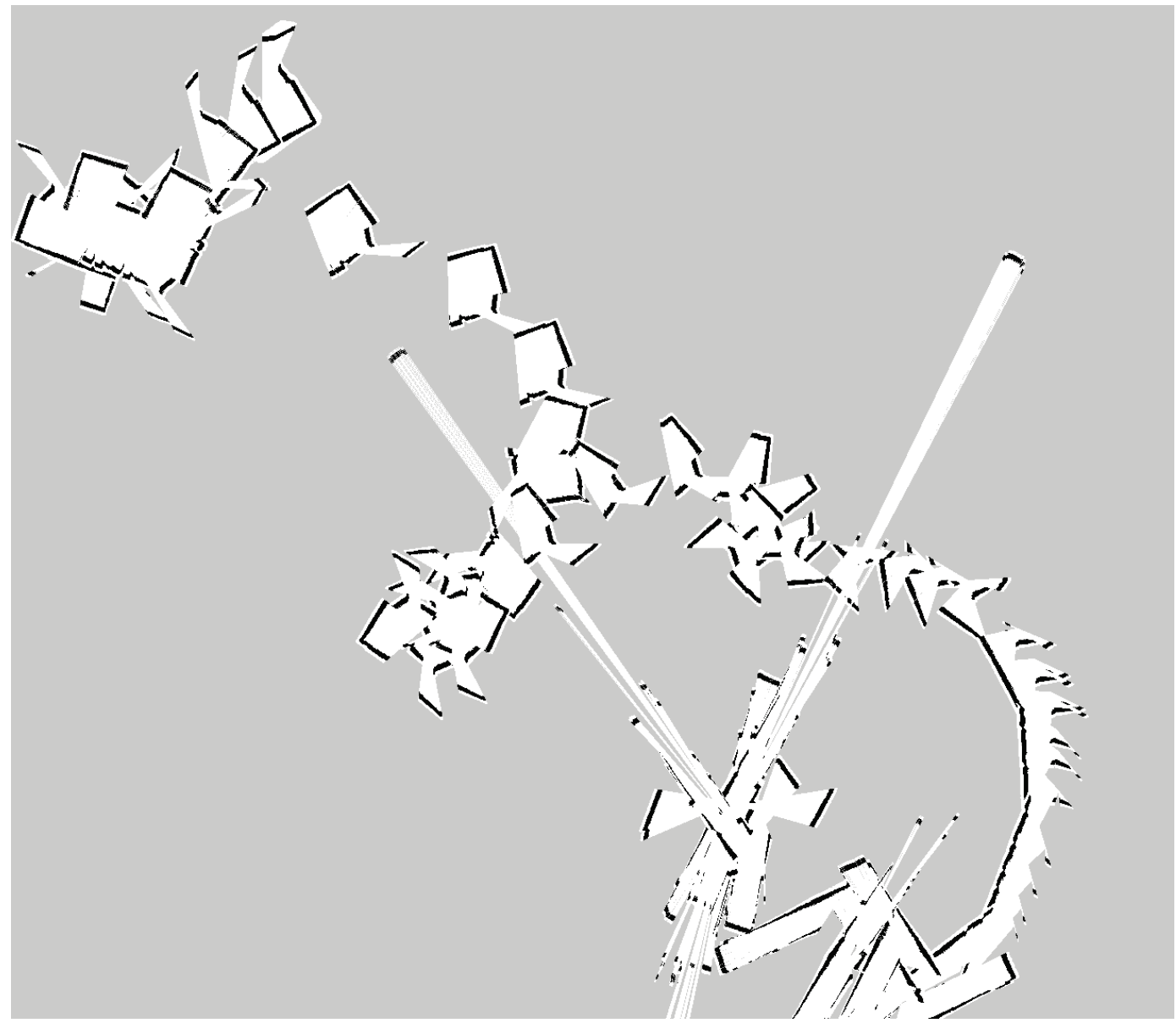


Figure 26. Generated CoreSLAM map of foyer.

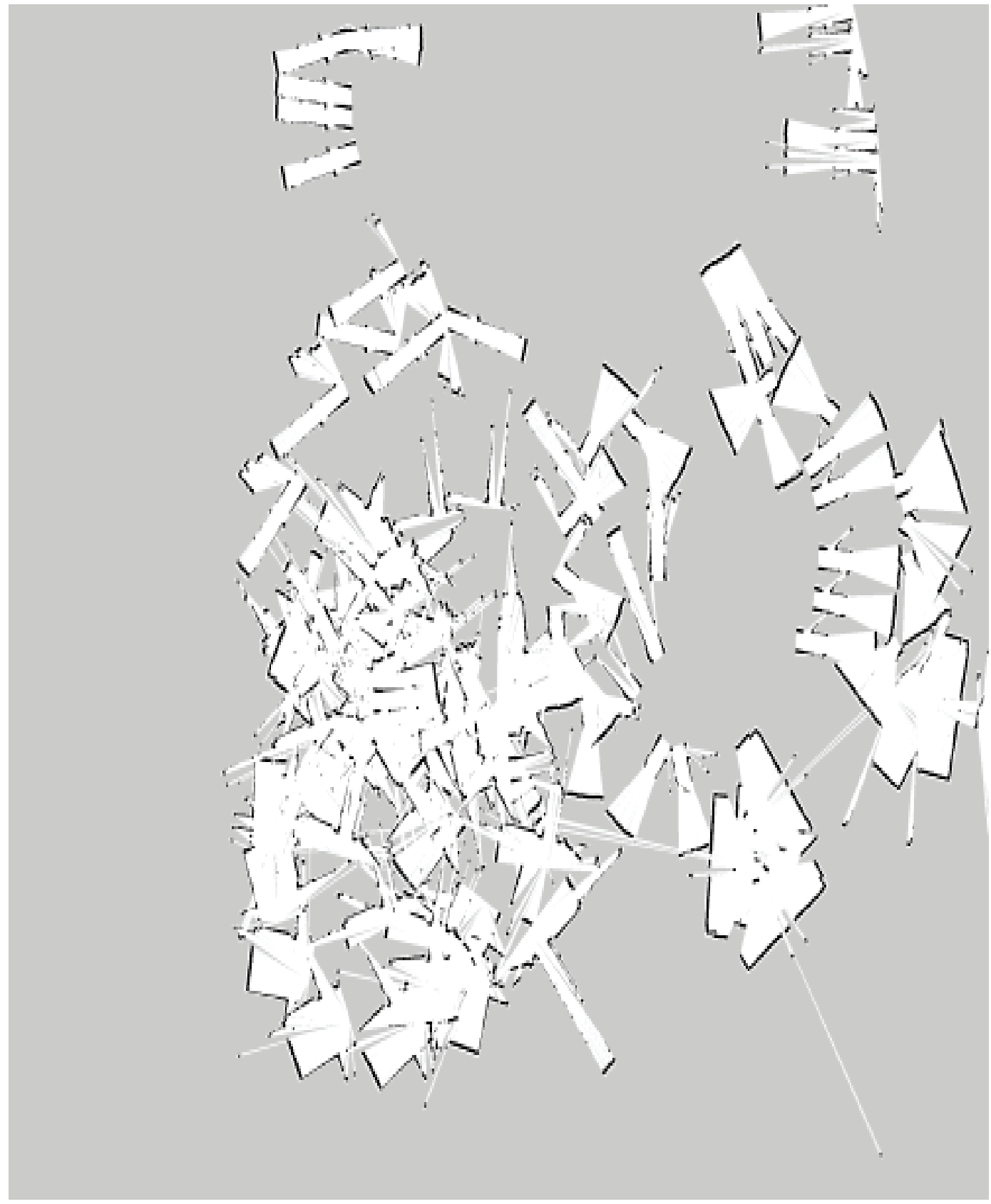

\subsubsection{Gmapping}

Figure 27 depicts the generated map from the Gmapping for the basement and Figure 28 the generated map of the foyer. Gmapping performed poorly on the physical robot, and the produced maps were of poor quality and unrecognizable compared to the ground truth maps. 
Figure 27. Generated Gmapping map of basement.

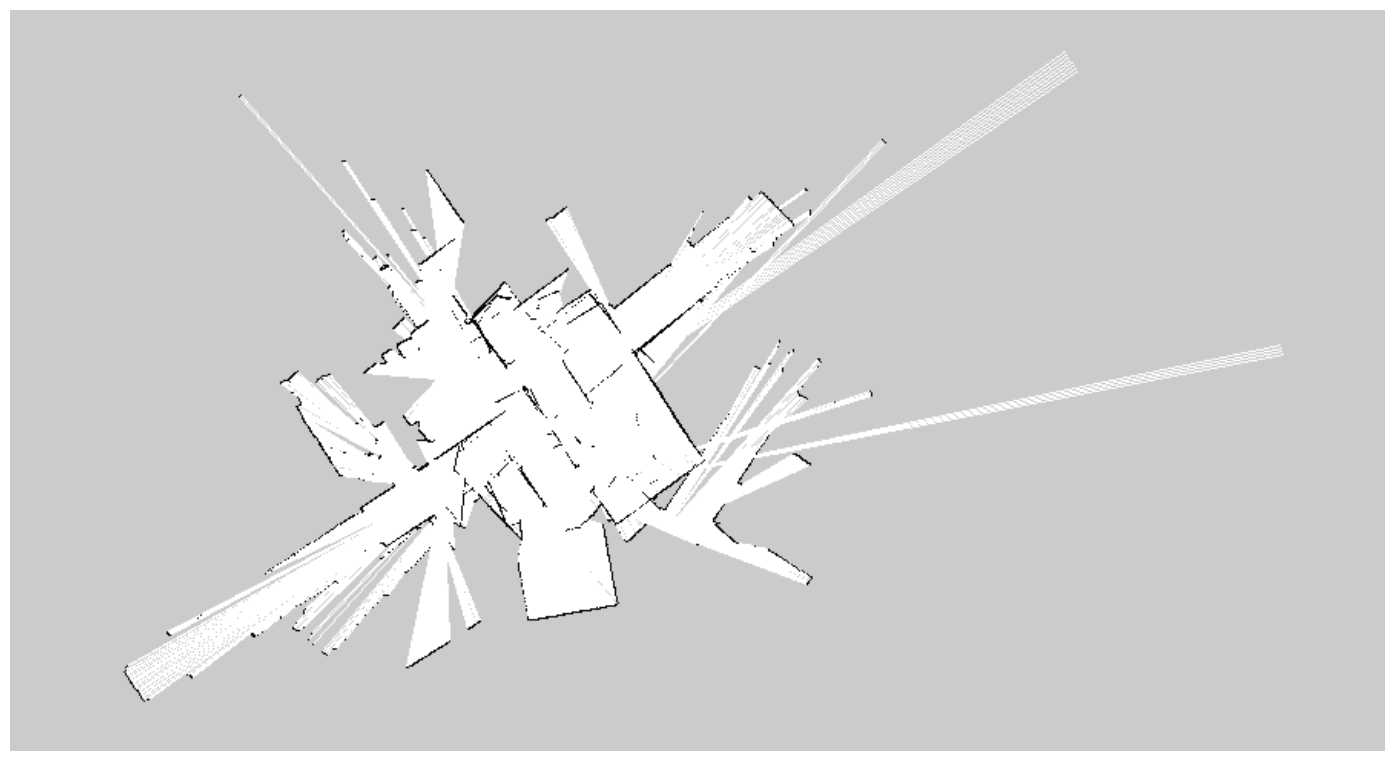

\subsubsection{HectorSLAM}

Figure 29 depicts the generated map from Hector SLAM for the basement, and Figure 30 is the generated map of the foyer. HectorSLAM performed efficiently on the physical robot, and the produced maps were good quality and recognizable, when compared to the ground truth maps. The generated map of the basement was somewhat distorted, but each of the four rooms and the hallway were identifiable.

\subsection{Comparison of ground truth map and generated map}

Hector SLAM produced a better map than CoreSLAM and Gmapping. Figure 31 shows the generated Hector SLAM map and actual photographs of the test site. Doors, windows, tables, chairs, and file cabinets can be identified in the generated map. Most of the major corners of the Hector SLAM map are recognizable. The Gmapping generated map of the foyer had some recognizable features, but CoreSLAM had no recognizable features.

The map in the center is the generated map and the color images are actual photos of the foyer, breakroom, and mailroom. The generated map in the center has labels pointing to open door, tables and chairs, walls, and an unexplored area. The open door label points to an area where the Explorer did not travel, but the laser scans down the hallway off the foyer. Had the door been closed it would have scanned as a wall. The table and chairs label shows the dots on the floor where the table and chair legs are. 
Figure 28. Generated Gmapping SLAM map of foyer.

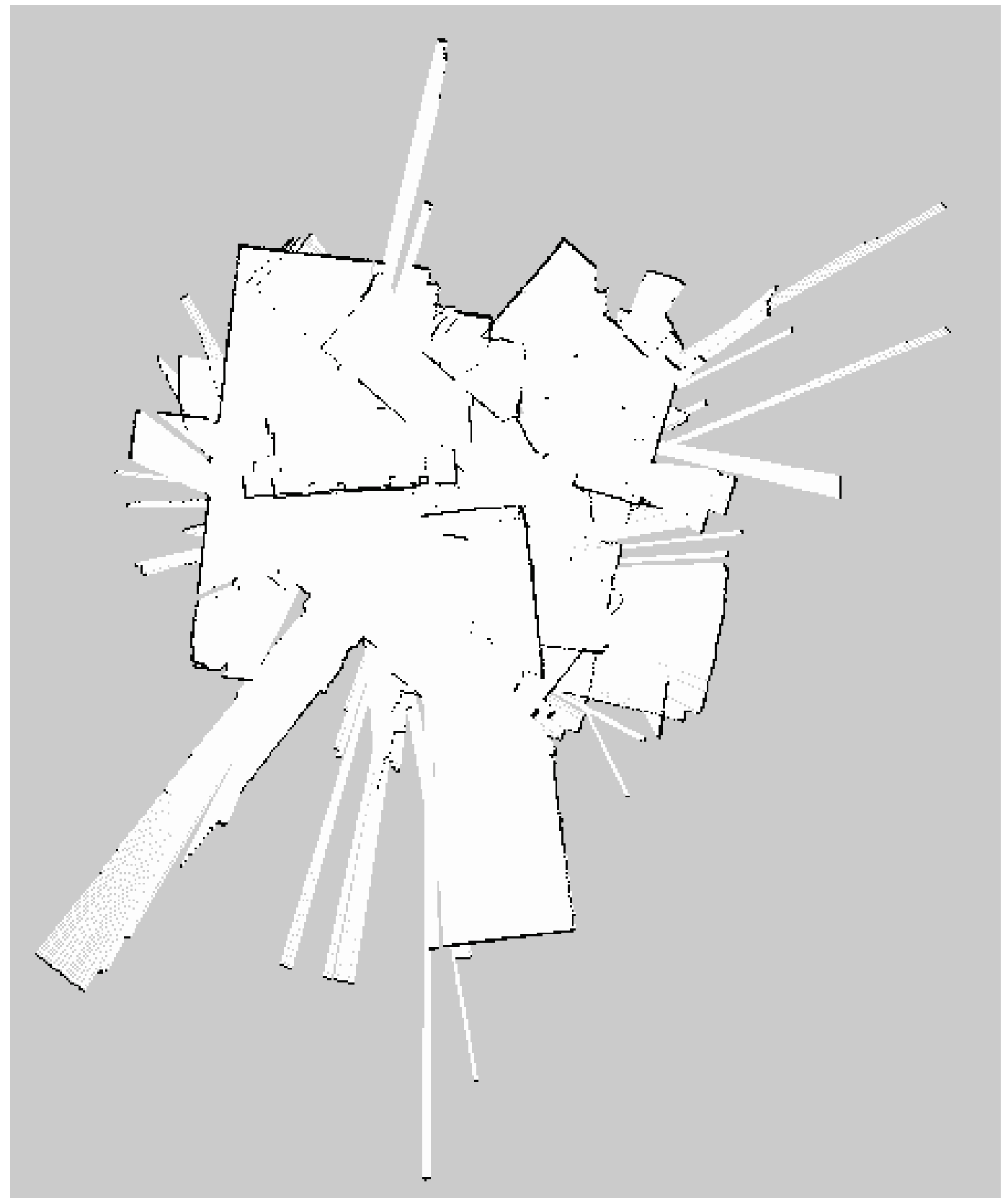

The wall label is an area where there are actually walls, cabinets, or door where the laser scanner cannot scan. The unexplored area, the gray area, is an area in the mailroom where the laser scan did not scan. 
Figure 29. Generated Hector SLAM map of basement.

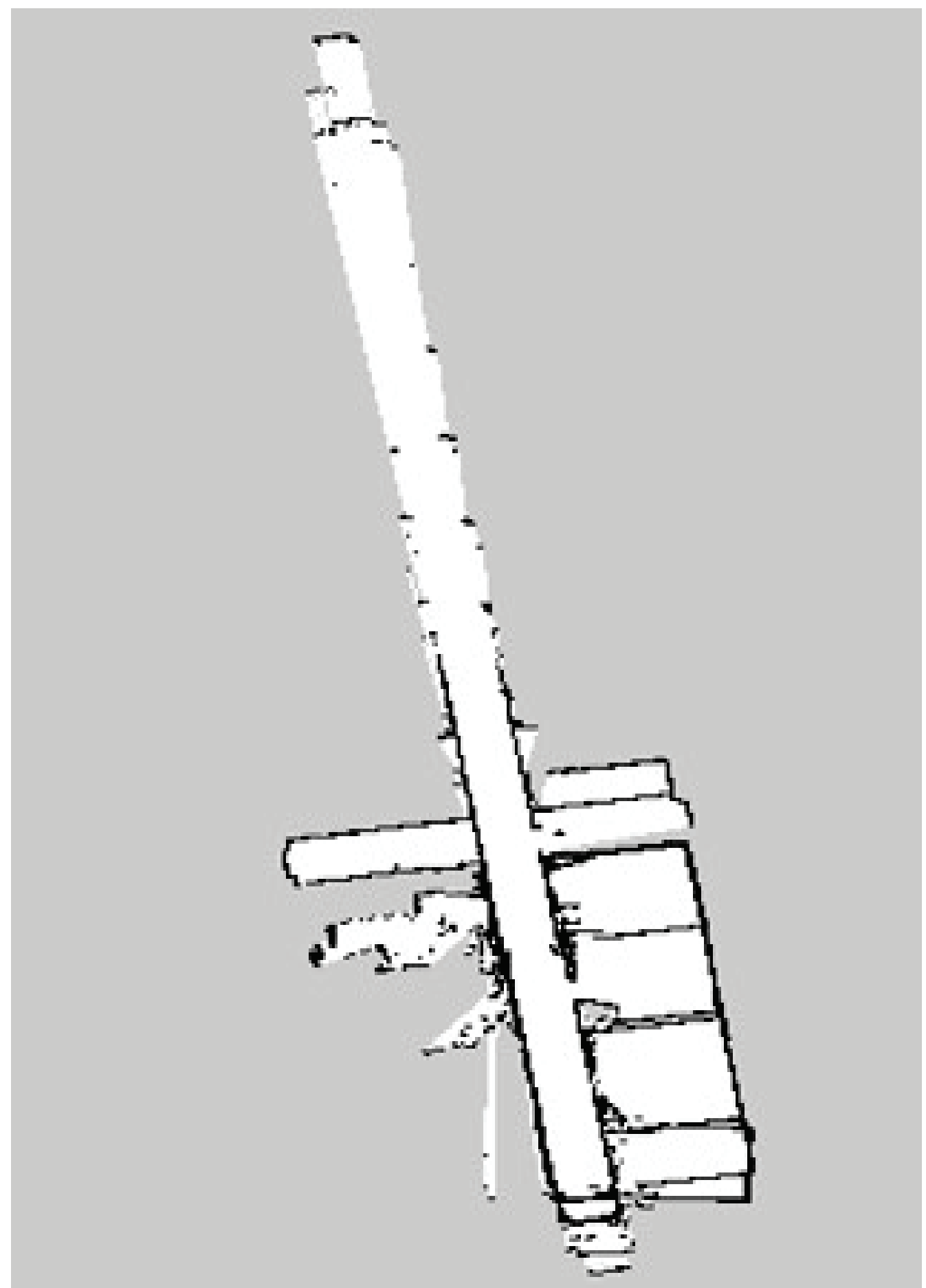

The map in the center of Figure 32 is the generated map of the basement and the color images are actual photos of the basement, consisting of a hallway and four small rooms. The generated map in the center has labels pointing to empty rooms, the hallway and a breakroom not traversed by the robot but scanned by the laser scanner. As depicted in Figure 31, the 
rooms are small and the hallway narrow, and the lighting varies from light to dark. Too much light could affect the scan, so paper and blinds were placed over the window to control the amount of light. I believe the smallness of rooms and the narrowness of the hallway had an effect on the quality of the map.

Figure 30. Generated Hector SLAM map of foyer.

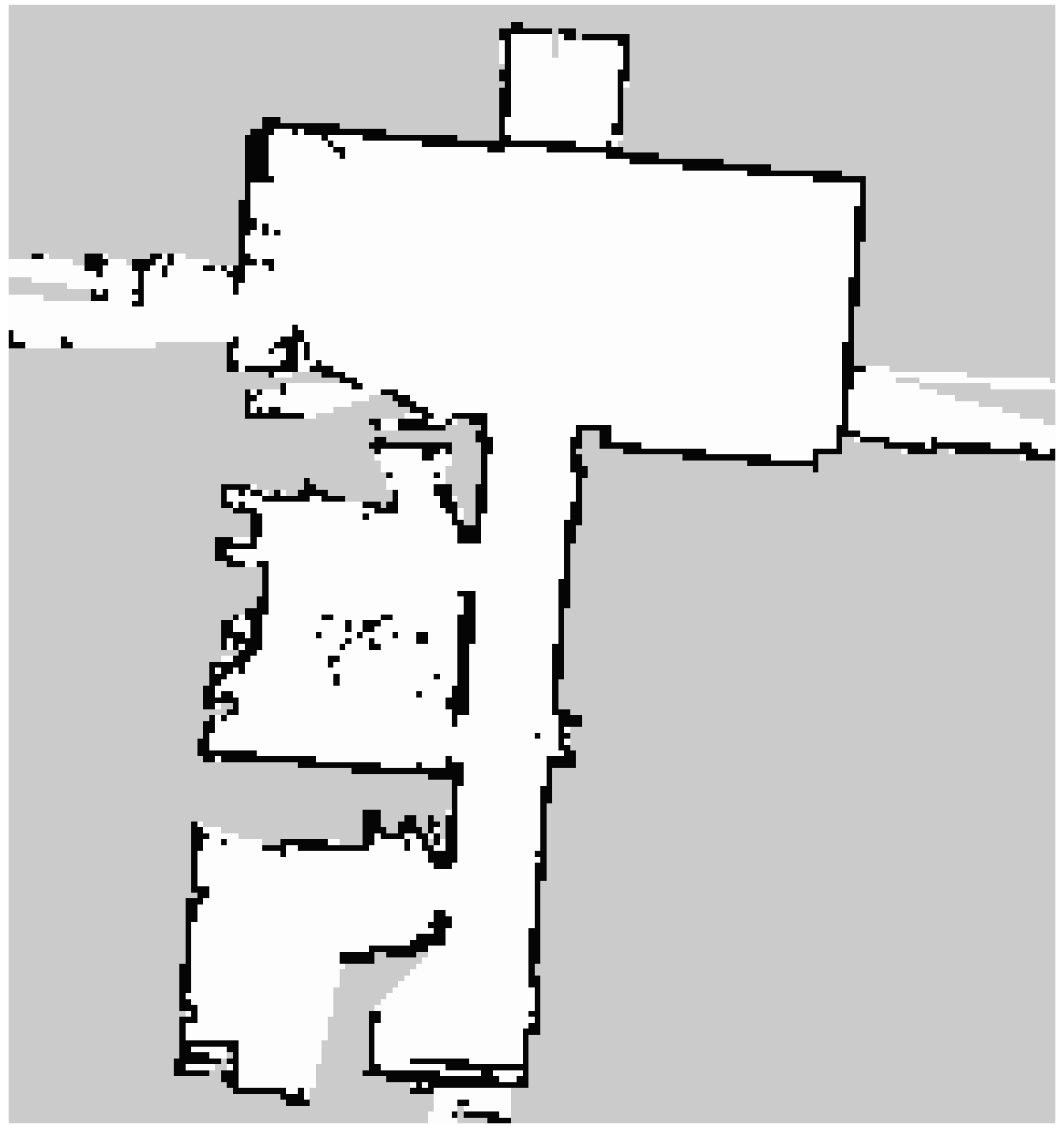


Figure 31. Generated Hector Slam map and pictures of actual foyer.

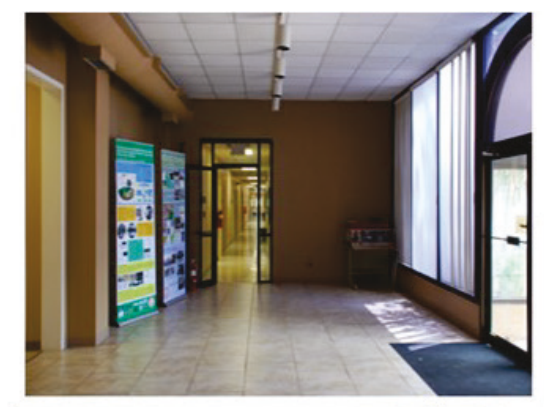

\section{Open door}

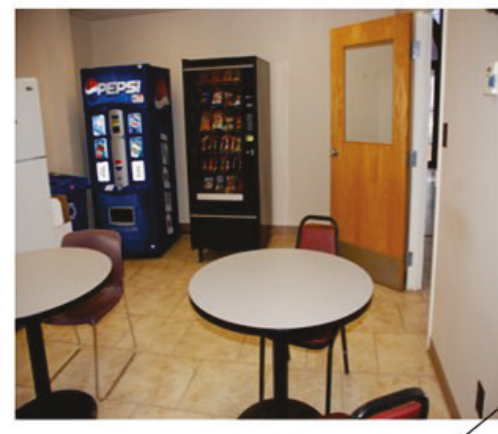

Table and chairs
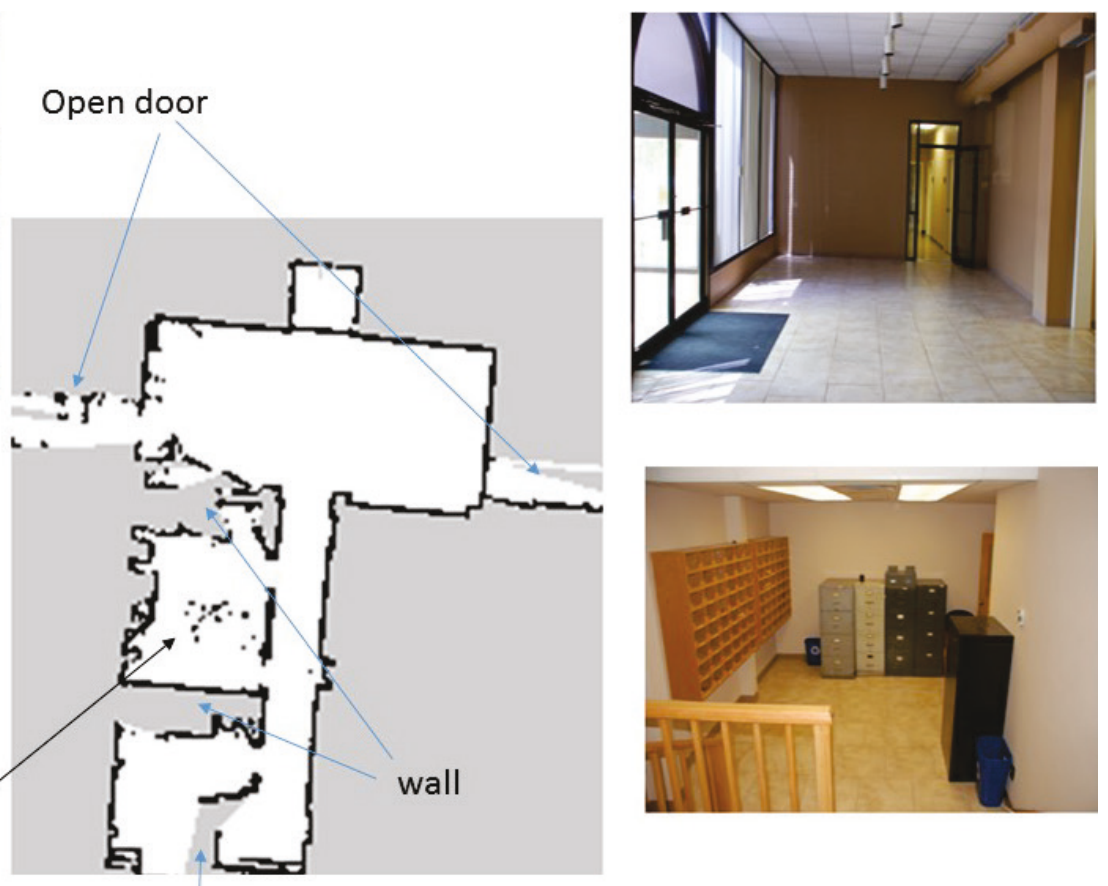

Unexplored area

Figure 32. Generated Hector Slam map and pictures of actual basement.
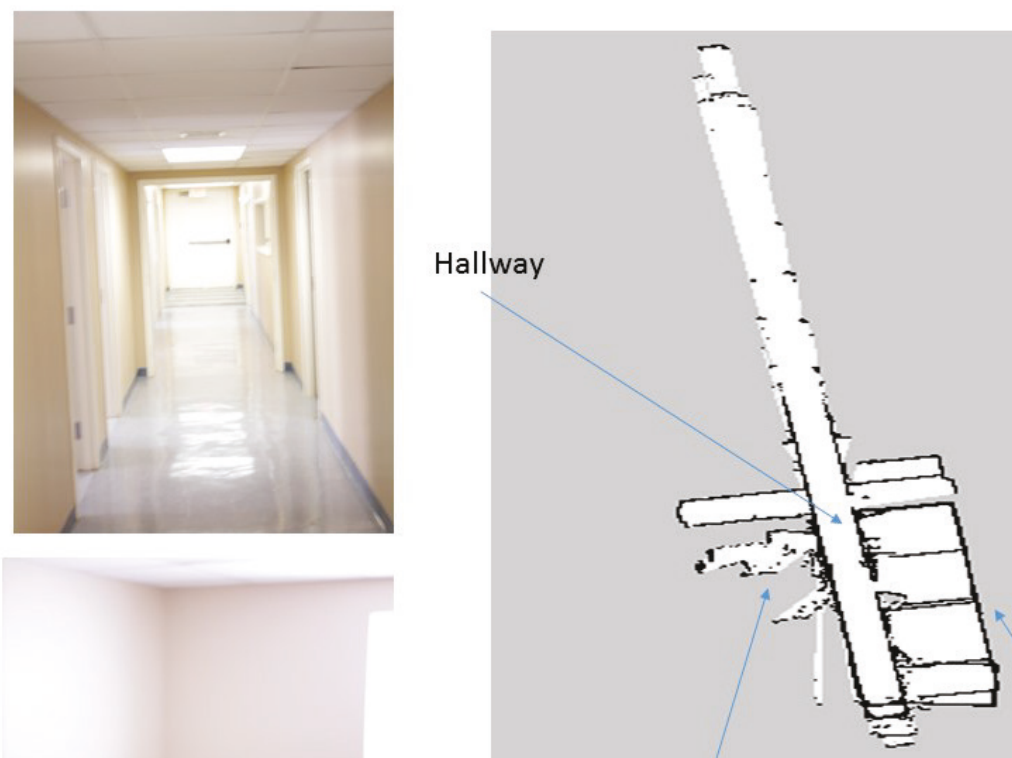

Empty rooms
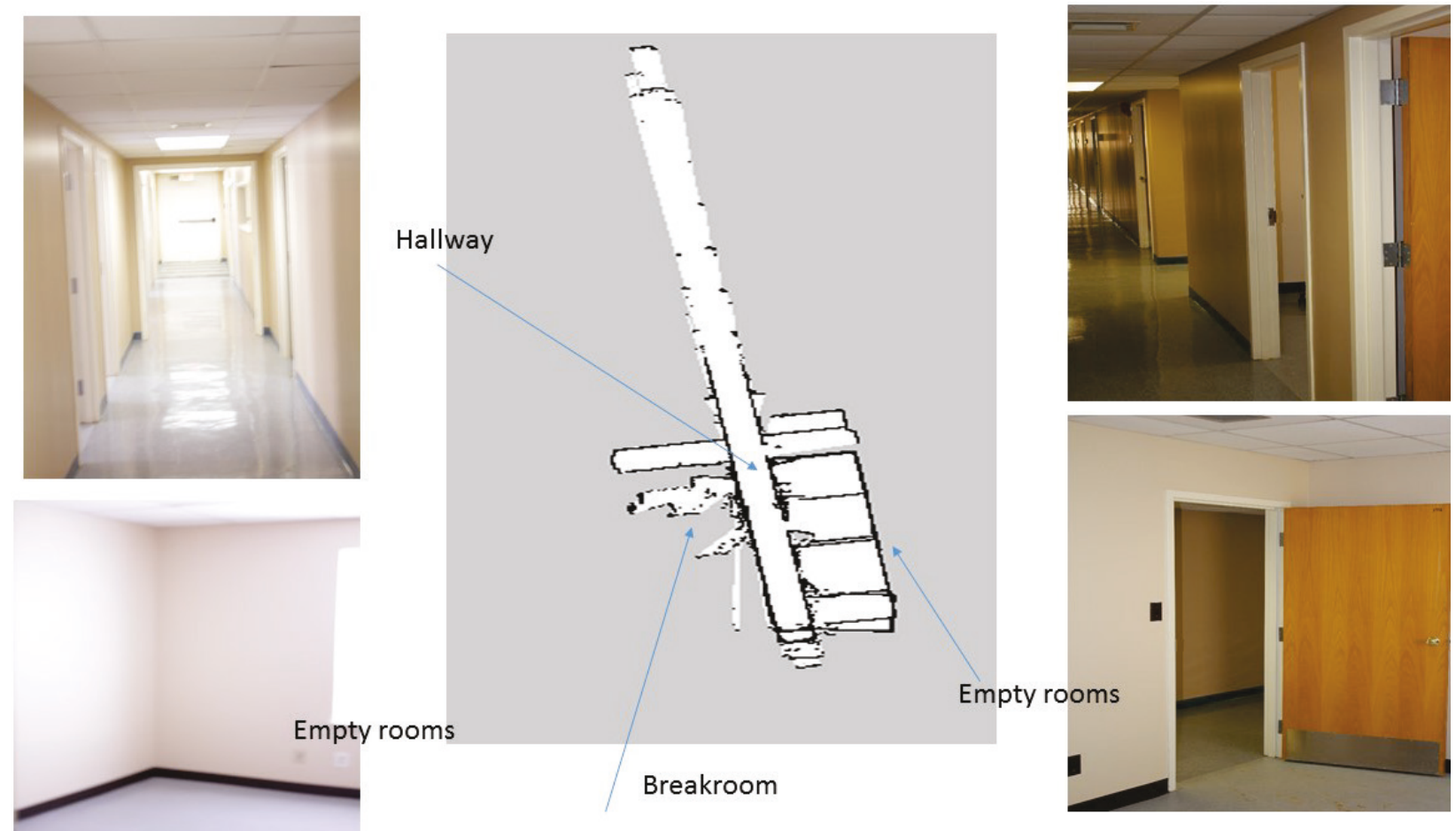


\subsection{Results}

This chapter provided physical testing of the Explorer in the foyer area and the basement. The Explorer performed better in the foyer, and a higher quality map was generated. This Hector SLAM and Coroware Explorer research increases the ability of the ERDC to explore and map unknown areas and aids in increasing the level of autonomy of current unmanned vehicles used during the exploration of unknown areas. This implementation and testing provides further validation of the Hector Slam algorithm for real-world application. 


\section{Localization and Pathfinding}

This chapter localizes the Explorer to a position in the generated gridbased map produced by Hector Slam in Chapter 5 using the Adaptive Monte Carlo Localization Package (AMCL) for a robot moving in 2-D. AMCL produces the positon of the moving robot in the map. Several maps are produced at resolutions with three different physical locations. The results of the robot's path-finding ability with $\mathrm{A}^{*}$ algorithm package was collected for a path between two points, in both the generated SLAM map and the physical site.

\subsection{A* pathfinding package}

The goal of this package is for the Explorer to create a path based on a grid-based map produced by the mobile Explorer. Several maps are generated at varying resolutions of $100 \times 100,200 \times 200,300 \times 300$, $500 \times 500$, and $1000 \times 1000$ of 3 different test sites. The Explorer travels around the map from start node A, provided by the AMCL package, and to destination node $\mathrm{B}$, provided by the user and stored and passed to the $\mathrm{A}^{*}$ package. A path is produced by the $\mathrm{A}^{*}$ algorithm, and then the path is timed and traversed.

\subsection{Requirements}

The mobile robot must be able to localize itself in the grid-based map produced by HectorSLAM and traverse a path produced by the $\mathrm{A}^{*}$ algorithm. The package takes as input a grid-base map, a start location, and a destination location. The robot must travel the path produced by $\mathrm{A}^{*}$. The robot must also traverse the map, storing a start location, and travel to a destination. The actual distance between the destination point $B$ of the SLAM map and point B of the physical map was measured, recorded, and analyzed.

\subsection{Analysis and design}

The analysis and design phase consists of the analysis and design to localize the Explorer to a grid-based map and produce a path in the gridbased map. The Corobot $A^{*}$ package allows the Explorer to traverse a path in a grid-base map autonomously. The $\mathrm{A}^{*}$ Package has the following steps.

1. Take as input a grid-based map produced with HectorSLAM. 
2. Convert the map to an array of os and $1 \mathrm{~s}$ based on map resolution. $\mathrm{o}$ is free. 1 is occupied.

3. Tele-operate the mobile robot to localize to the map and get the current positon of the robot.

4. Store the current position as the start location.

5. Enter the destination position.

6. Calculate the path.

7. Convert the start and destination position to cells in the map.

8. Set start time for path traversal.

9. Mobile robot travels autonomously to destination.

10. Set end time for path traversal.

11. Calculate time to traverse path.

12. Measure the distance between the starting and ending points.

13. Repeat process on maps of varying resolution.

14. Collect results on pathfinding at each resolution.

\subsubsection{AMCL overview}

AMCL is a ROS package designed to provide a mobile robot its pose while traversing a known map. AMCL is a probabilistic localization system for a robot moving in 2-D (AMCL site). AMCL implements the adaptive (or KLD-sampling) Monte Carlo approach as described by Dieter Fox (AMCL site). AMCL takes as input a laser-based map and publishes the mobile robot's estimated pose in the map. AMCL subscribes to topics in Table 4.

Table 4. Subscribed topic.

\begin{tabular}{|l|l|}
\hline scan & sensor_msgs/LaserScan \\
\hline $\mathrm{tf}$ & $\mathrm{tf} / \mathrm{tf} \_m e s s a g e s$ \\
\hline initialpose & geometry_msgs/PosWithCovarianceStamped \\
\hline Map & Navigation_msgs \\
\hline
\end{tabular}

AMCL publishes the amcl_pose, which is the pose of the mobile robot against the known map.

\subsubsection{Corobot_A* package}

The Corobot $\mathrm{A}^{*}$ Package implements the $\mathrm{A}^{*}$ algorithm with the Manhattan Cost function. The package uses the robot pose, an $\mathrm{x}$ and $\mathrm{y}$ position in the map, provided by AMCL, to use as the start location for the path, and the user provides a random destination. The path between the start and 
destination location is calculated and provided. The robot then traverses the given path.

Three cost functions were considered: (1) the Manhattan Distance, (2) Euclidean Distance, and (3) the Chebyshev Distance.

The Manhattan Distance was implemented as the map is an $\mathrm{NxN}$ matrix, and four of the surrounding cells of the map were considered. The function is $\mathrm{F}=\mathrm{G}+\mathrm{H}$, where $\mathrm{G}$ is the total distance to the current position and $\mathrm{H}$ represents the cost.

\subsection{Implementation}

While the robot is traversing the area, a map is created of the explored area. The robot is then localized to the map using AMCL, explores the area, and displays its pose on the map. The robot can be given a destination position to travel using its current position as the start location.

\subsection{Testing}

Three test sites were used to evaluate the ability of the Explorer to localize itself in a generated SLAM map and travel from a start location to a destination location. For each site, two cones were used to mark points A and $\mathrm{B}$. The area around the cones were taped to marked points A and B. The area was traversed by the Explorer, and the SLAM map was generated. This map was used to localize the robot to the position A. The destination coordinate was entered into the $\mathrm{A}^{*}$ package, and the difference between the two $B$ points was measured and the time to traverse the path recorded.

\subsubsection{Test Site One}

Figure 33 depicts Test Site One, a 12-ft by 9-ft area that has walls on one side and two 6-ft tables as a wall for the $12-\mathrm{ft}$ side. The 9 -ft side has one 9 - $\mathrm{ft}$ table and a board to enclose one side, and the other 9 - $\mathrm{ft}$ side was a constructed wall. The $\mathrm{x}$-axis is marked and is determined to be the $\mathrm{x}$-axis by driving the Explorer around, and the $\mathrm{x}$ position of the pose data increased, which is the northing in the map. The y-axis is marked and is determined to be the y-axis by driving the Explorer around, and the $y$ position of the pose data increased. 
Figure 34 depicts Test Site One without the cones and positions A and B marked with tape and the SLAM generated map with the two black squares representing points A and B in the SLAM generated map.

Figure 33. Test Site One with cones denoting points A and B.

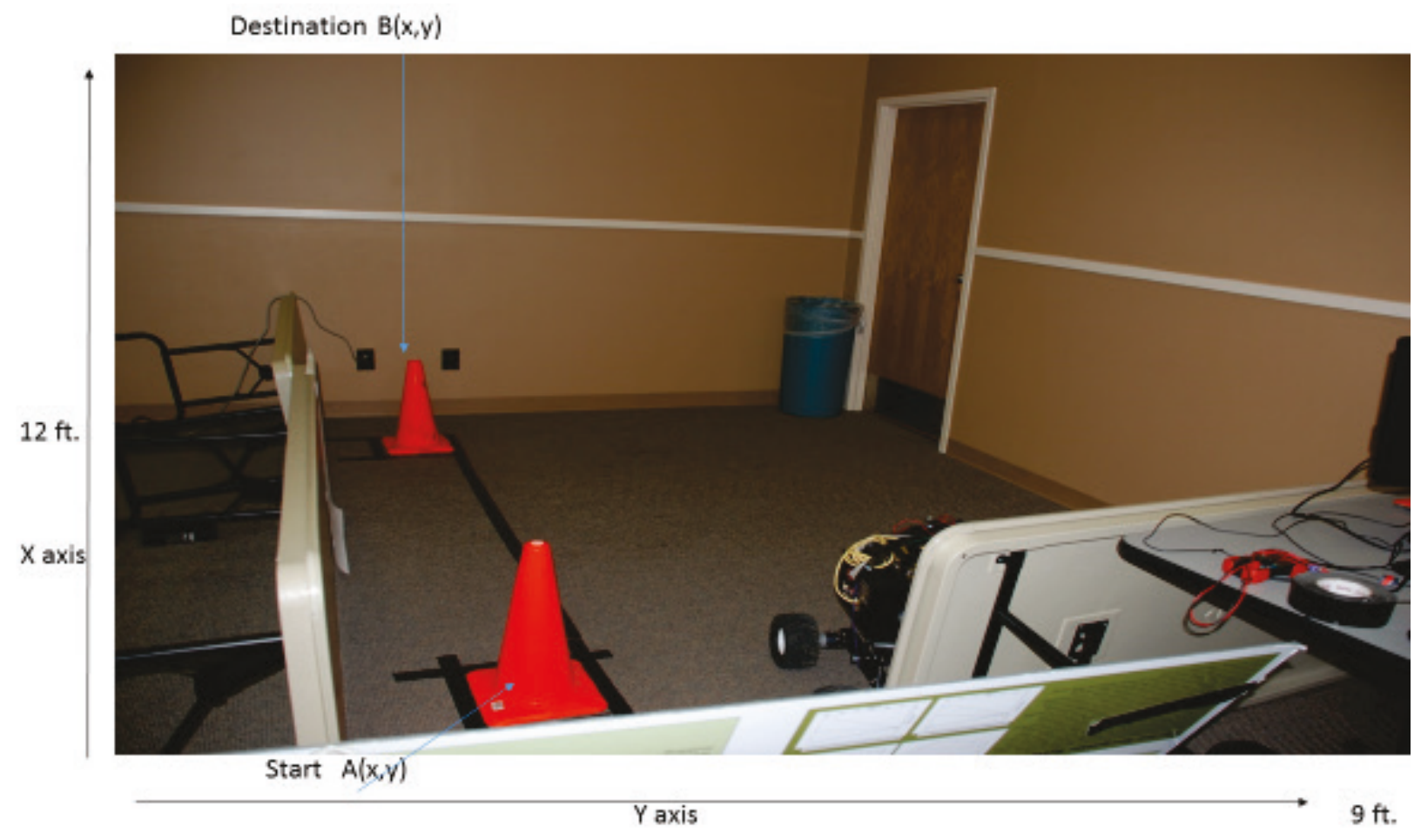

Figure 34. Test Site One without cones and the SLAM generated map.

Destination $\mathrm{B}(76,38)$
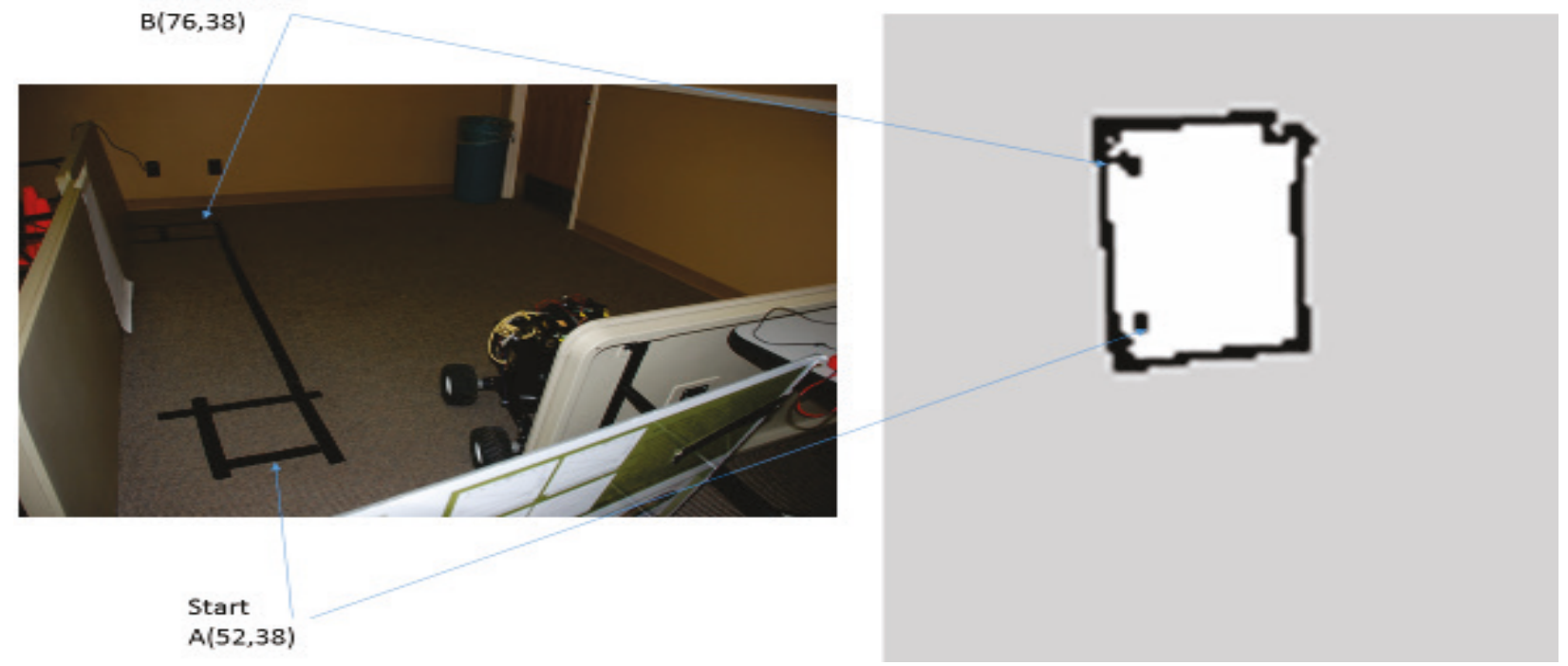
Next, the generated map is imported into GIMP 2 and scaled to the resolution of the map to gather the $\mathrm{x}$ and $\mathrm{y}$ positions for each of the points marked in the previous steps. The two points shown in the Figure 35 are for a map of resolution of $100 \times 100$. This step is repeated to gather the start and destination points for maps of resolution $200 \times 200,300 \times 300$, and $500 \times 500$.

Figure 35. GIMP view with imported SLAM map.

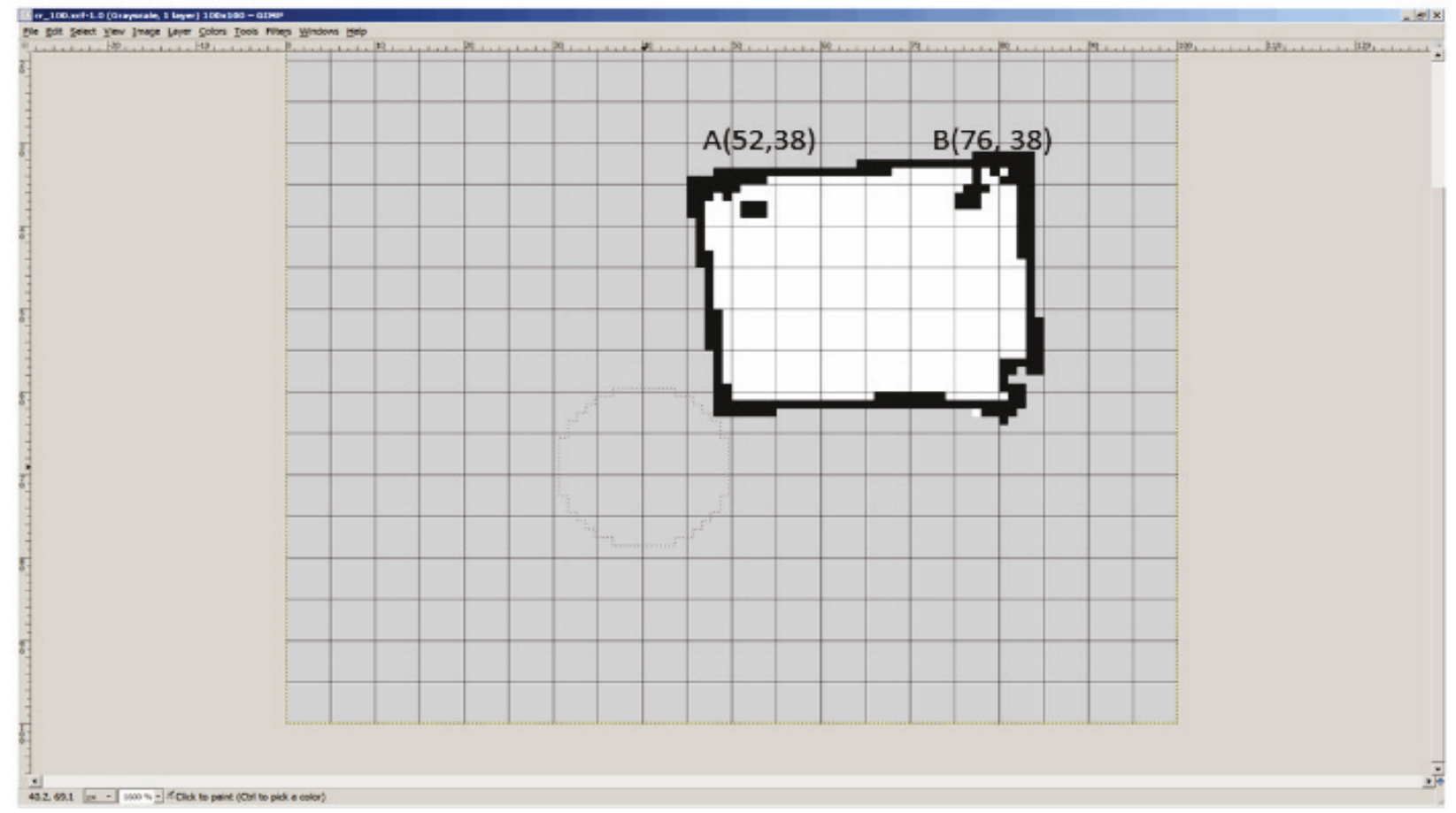

Next, the Explorer and laptop are started with the correct setup to use the AMCL Package and the generated map of the test site without the cones. The robot is placed in the A position of the test site, then localized to the map, and the $\mathrm{A}^{*}$ package is executed. The AMCL package provided a location of $(40,60)$ and provided a B position for the target location. Table 5 shows the results of these runs for a map of resolution $100 \times 100$. The distance from where the robot ended and the position of Point $B$ in the physical map is shown in the Distance column, and the time to traverse the path is shown in the Time column.

When entering the target location, the $\mathrm{A}^{*}$ package was executed until a B location close to the target destination B was found. Point $(76,38)$ was too large. Point $(65,40)$ and $(65,50)$ are feasible and very close to the target destination point. 
In the $100 \times 100$ resolution map, Table 5 , the error was from 7 to 16 in., with an average of $10.3 \mathrm{in}$. for the five runs. In the $200 \times 200$ resolution map, Table 6, the error was from o to $16 \mathrm{in}$., with an average of $9.9 \mathrm{in}$. for the five runs. In the $300 \times 300$ resolution map, Table 7 , the error was from o to $18 \mathrm{in}$., with an average of $9.2 \mathrm{in}$. for the five runs. In the $500 \times 500$, Table 8, resolution map, the error was from 2 to 18 in., with an average of 10.2 in. for the five runs. Overall, for Test Site One, the $300 \times 300$ resolution performed best.

Table 5. Results for $100 \times 100$ on Test Site One.

\begin{tabular}{|l|l|l|l|l|l|l|}
\hline & \multicolumn{2}{|c|}{ Generated Map } & \multicolumn{2}{c|}{ AMCL } & & \\
\hline & A & B & A & B & Time (sec.) & Distance (in.) \\
\hline Run 1 & $(52,38)$ & $(76,38)$ & $(60,40)$ & $(65,40)$ & 11.85 & 9.00 \\
\hline Run 2 & $(52,38)$ & $(76,38)$ & $(60,40)$ & $(65,40)$ & 15.51 & 12.00 \\
\hline Run 3 & $(52,38)$ & $(76,38)$ & $(60,40)$ & $(65,40)$ & 16.37 & 7.50 \\
\hline Run 4 & $(52,38)$ & $(76,38)$ & $(60,40)$ & $(65,40)$ & 13.50 & 16.00 \\
\hline Run 5 & $(52,38)$ & $(76,38)$ & $(60,50)$ & $(65,50)$ & 14.39 & 7.00 \\
\hline
\end{tabular}

Table 6. Results $200 \times 200$ on Test Site One.

\begin{tabular}{|l|l|l|l|l|l|l|}
\hline & \multicolumn{2}{|c|}{ Generated Map } & \multicolumn{2}{c|}{ AMCL } & & \\
\hline & A & B & A & B & Time (sec.) & Distance (in.) \\
\hline Run 1 & $(154,141)$ & $(177,148)$ & $(90,110)$ & $(90,110)$ & 13.36 & 0 \\
\hline Run 2 & $(154,141)$ & $(177,148)$ & $(90,110)$ & $(90,110)$ & 14.20 & 0 \\
\hline Run 3 & $(154,141)$ & $(177,148)$ & $(90,110)$ & $(90,110)$ & 14.72 & 17 \\
\hline Run 4 & $(154,141)$ & $(177,148)$ & $(90,110)$ & $(90,116)$ & 16.35 & 16 \\
\hline Run 5 & $(154,141)$ & $(177,148)$ & $(90,110)$ & $(90,116)$ & 13.42 & 16 \\
\hline
\end{tabular}

In Test Site One, when entering the generated map's point B, the robot could not find point B. The robot would try to travel up the wall and would have to be stopped. This led to finding a point $\mathrm{B}$ close to the Point B on the floor to enter. This was possibly caused by the area being too small, and Point A and Point B being close together in the physical map. As we move to Test Site Two, a larger area was chosen. 
Table 7. Results for $300 \times 300$ on Test Site One.

\begin{tabular}{|l|l|l|l|l|l|l|}
\hline & \multicolumn{2}{|c|}{ Generated Map } & \multicolumn{2}{c|}{ AMCL } & & \\
\hline & A & B & A & B & Time (sec.) & Distance (in.) \\
\hline Run 1 & $(155,140)$ & $(180,146)$ & $(150,150)$ & $(150,156)$ & 20.57 & 16 \\
\hline Run 2 & $(155,140)$ & $(180,146)$ & $150,150)$ & $(150,156)$ & 15.01 & 18 \\
\hline Run 3 & $(155,140)$ & $(180,146)$ & $150,150)$ & $(150,156)$ & 14.46 & 4 \\
\hline Run 4 & $(155,140)$ & $(180,146)$ & $150,150)$ & $(150,156)$ & 13.52 & 8 \\
\hline Run 5 & $(155,140)$ & $(180,146)$ & $150,150)$ & $(150,156)$ & 15.70 & 0 \\
\hline
\end{tabular}

Table 8. Results for $500 \times 500$ on Test Site One.

\begin{tabular}{|l|l|l|l|l|l|l|}
\hline & \multicolumn{2}{|c|}{ Generated Map } & \multicolumn{2}{c|}{ AMCL } & & \\
\hline & A & B & A & B & Time (sec.) & Distance (in.) \\
\hline Run 1 & $(250,243)$ & $(250,241)$ & $(250,250)$ & $(250,256)$ & 8.87 & 14 \\
\hline Run 2 & $(250,243)$ & $(250,241)$ & $(250,250)$ & $(250,256)$ & 9.83 & 2 \\
\hline Run 3 & $(250,243)$ & $(250,241)$ & $(240,250)$ & $(250,257)$ & 9.33 & 5 \\
\hline Run 4 & $(250,243)$ & $(250,241)$ & $(250,250)$ & $(250,256)$ & 8.99 & 12 \\
\hline Run 5 & $(250,243)$ & $(250,241)$ & $(250,250)$ & $(250,256)$ & 8.72 & 18 \\
\hline
\end{tabular}

\subsubsection{Test Site Two}

Test Site Two is an area approximately $15 \mathrm{ft}$ by $24 \mathrm{ft}$ with bookshelves, several desks, a copy machine, a garbage can, and several small file cabinets, (Figure 36). The hallway is blocked by a piece of board to make a wall. The map was generated for Test Site Two with Hector SLAM with the perimeter of the two cones taped on the floor in order to mark points $A$ $(\mathrm{x}, \mathrm{y})$ and $\mathrm{B}(\mathrm{x}, \mathrm{y})$ in the actual test site.

Next, the generated maps are imported into GIMP 2 and scaled to resolution size. For Test Site Two, the resolutions tested were $200 \times 200$, $300 \times 300,500 \times 500$, and $1000 \times 1000$. Resolution $100 \times 100$ produced a poor quality map and was not on the canvas. In Figure 37, Point A is the start location, and Point B is the destination location.

Next, the Explorer and laptop are started with the correct setup to use the AMCL Package and the generated map of the test site without the cones. 
Figure 36. Front and rear view of Test Site Two with cones.

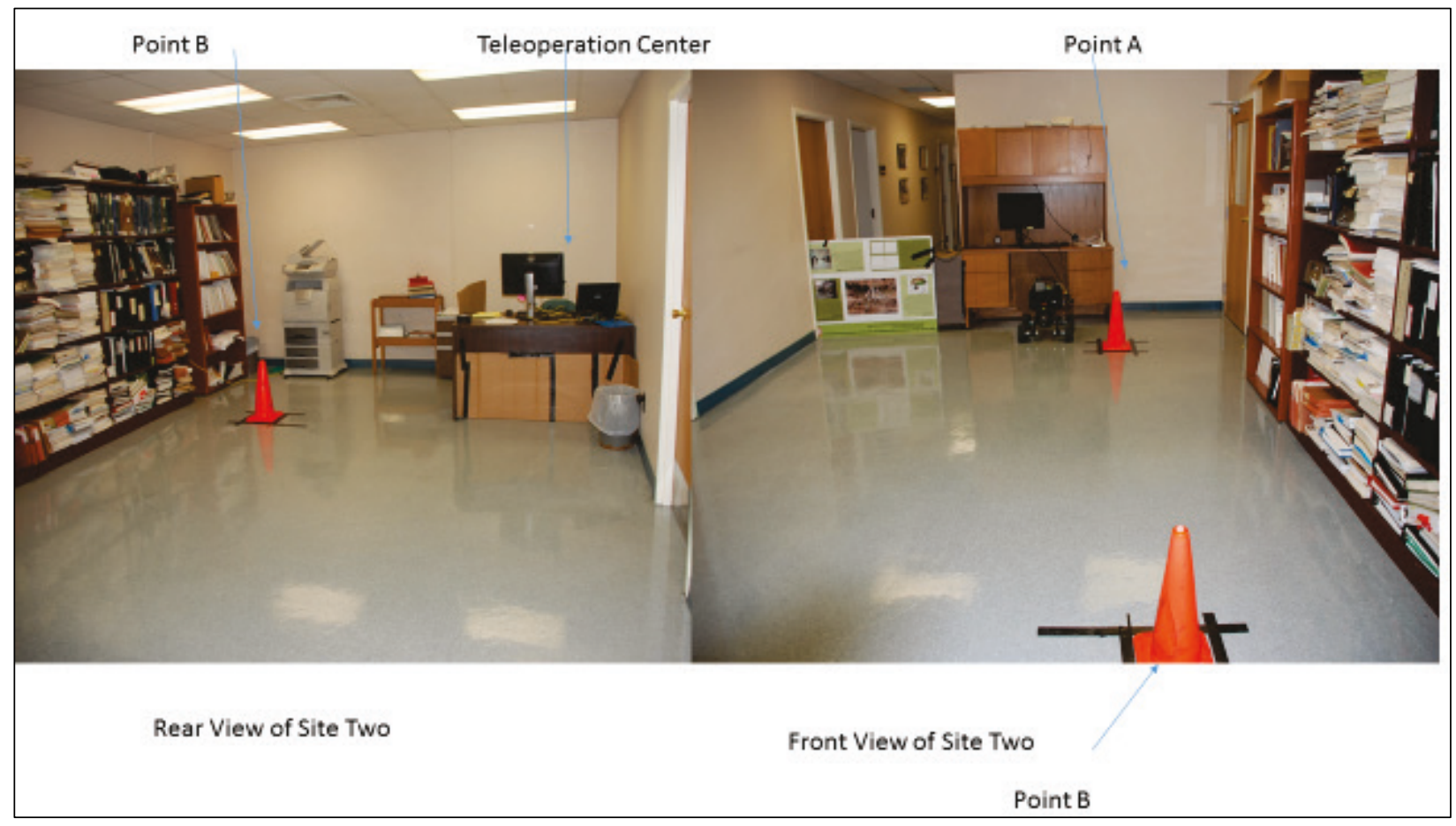

Figure 37. Test Site Two resolution $300 \times 300$.

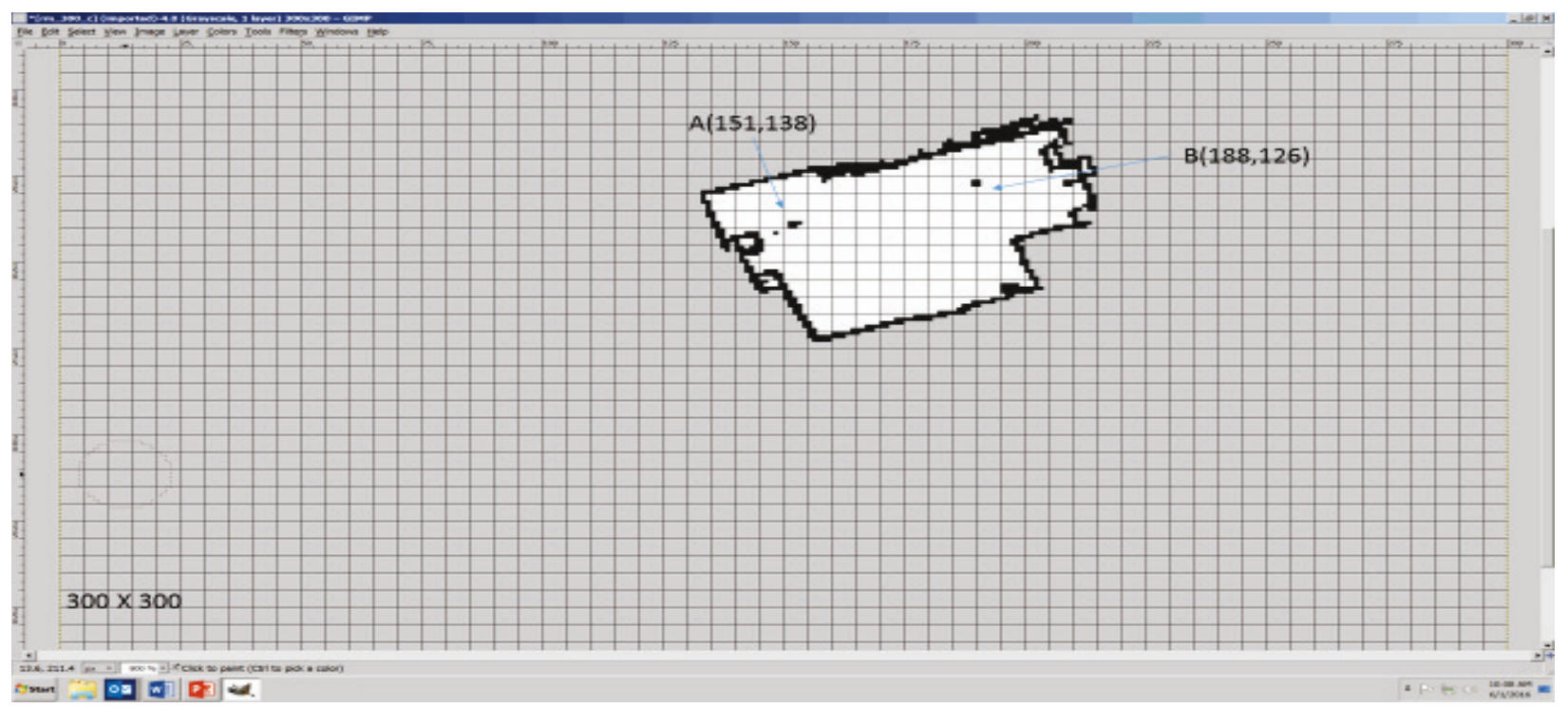

The Explore was placed in the start position A of the test site, then localized to the map, and the $A^{*}$ package was executed. The AMCL package provided the starting point $\mathrm{A}(\mathrm{x}, \mathrm{y})$, and the user provided $\mathrm{B}(\mathrm{x}, \mathrm{y})$ position for the destination location. No results were collected for Resolution $1000 \times 1000$. Each time the $A^{*}$ Package was executed, the package had a memory core dump. 
In the $200 \times 200$ resolution map, Table 9 , the error was from o to 48 in., with an average of $27.6 \mathrm{in}$. for the five runs. In the $300 \times 300$ resolution map, Table 10, the error was from o to $12 \mathrm{in}$., with an average of $2.8 \mathrm{in}$. for the five runs. In the $500 \times 500$ resolution map, Table 11 , the error was from $\mathrm{o}$ to $12 \mathrm{in}$., with an average of $2.8 \mathrm{in}$. for the five runs. Overall, for Test Site 2 , the $300 \times 300$ and $500 \times 500$ resolution performed best.

Table 9. Results for $200 \times 200$ on Test Site Two.

\begin{tabular}{|l|l|l|l|l|l|l|}
\hline & \multicolumn{2}{|c|}{ Generated Map } & AMCL & Entered & \multicolumn{2}{l|}{} \\
\hline & A & B & A & B & Time (sec.) & Distance (in.) \\
\hline Run 1 & $(107,92)$ & $(145,99)$ & $(100,100)$ & $(115,100)$ & 21.67 & 0 \\
\hline Run 2 & $(107,92)$ & $(145,99)$ & $(100,100)$ & $(115,100)$ & 21.03 & 30 \\
\hline Run 3 & $(107,92)$ & $(145,99)$ & $(100,100)$ & $(115,100)$ & 20.54 & 24 \\
\hline Run 4 & $(107,92)$ & $(145,99)$ & $(100,100)$ & $(115,100)$ & 22.23 & 36 \\
\hline Run 5 & $(107,92)$ & $(145,99)$ & $(100,100)$ & $(115,100)$ & 23.48 & 48 \\
\hline
\end{tabular}

Table 10.Results for $300 \times 300$ on Test Site Two.

\begin{tabular}{|l|l|l|l|l|l|l|}
\hline & \multicolumn{2}{|c|}{ Generated Map } & AMCL & Entered & \multicolumn{2}{l|}{} \\
\hline & A & B & A & B & Time (sec.) & Distance (in.) \\
\hline Run 1 & $(151,138)$ & $(188,126)$ & $(150,150)$ & $(150,164)$ & 19.70 & 1 \\
\hline Run 2 & $(151,138)$ & $(188,126)$ & $(140,150)$ & $(140,164)$ & 19.51 & 12 \\
\hline Run 3 & $(151,138)$ & $(188,126)$ & $(150,150)$ & $(150,164)$ & 19.16 & 1 \\
\hline Run 4 & $(151,138)$ & $(188,126)$ & $(150,150)$ & $(150,164)$ & 19.20 & 0 \\
\hline Run 5 & $(151,138)$ & $(188,126)$ & $(140,150)$ & $(140,164)$ & 19.56 & 0 \\
\hline
\end{tabular}

Table 11. Results for $500 \times 500$ on Test Site Two.

\begin{tabular}{|l|l|l|l|l|l|l|}
\hline & \multicolumn{2}{|c|}{ Generated Map } & AMCL & Entered & \multicolumn{2}{l}{} \\
\hline & A & B & A & B & Time (sec.) & Distance (in.) \\
\hline Run 1 & $(256,241)$ & $(294,241)$ & $250,250)$ & $(264,250)$ & 21.04 & 2 \\
\hline Run 2 & $(256,241)$ & $(294,241)$ & $(250,250)$ & $(264,240)$ & 20.53 & 12 \\
\hline Run 3 & $(256,241)$ & $(294,241)$ & $(240,250)$ & $(264,250)$ & 19.34 & 0 \\
\hline Run 4 & $(256,241)$ & $(294,241)$ & $(240,250)$ & $(264,250)$ & 19.63 & 0 \\
\hline Run 5 & $(256,241)$ & $(294,241)$ & $(240,250)$ & $(264,250)$ & 19.71 & 0 \\
\hline
\end{tabular}


In this set of tests, it is obvious that changing the resolution decreases some of the error. Creating a map of resolution $100 \times 100$ was unsuccessful. The map that was produced was of poor quality. As in Test Site One, the entered Point $\mathrm{B}$ was tweaked by driving the robot around to find a feasible Point $\mathrm{B}$. The Point $\mathrm{B}$ of the generated map and the one collected were extremely different, but once Point B was found, the error was low.

\subsubsection{Test Site Three}

The map was generated for Test Site Three, an area approximately $15 \mathrm{ft}$ by $24 \mathrm{ft}$ with Hector SLAM with the perimeter of the two cones taped on the floor in order to mark Points $\mathrm{A}(\mathrm{x}, \mathrm{y})$ and $\mathrm{B}(\mathrm{x}, \mathrm{y})$ in the actual test site. Test Site Three is the same room as Test Site Two, but the room was rearranged. The copy machine was moved, and the desk was moved from one wall to another. Figure 38 depicts Test Site Three.

Figure 38. Test Site Three with cone.
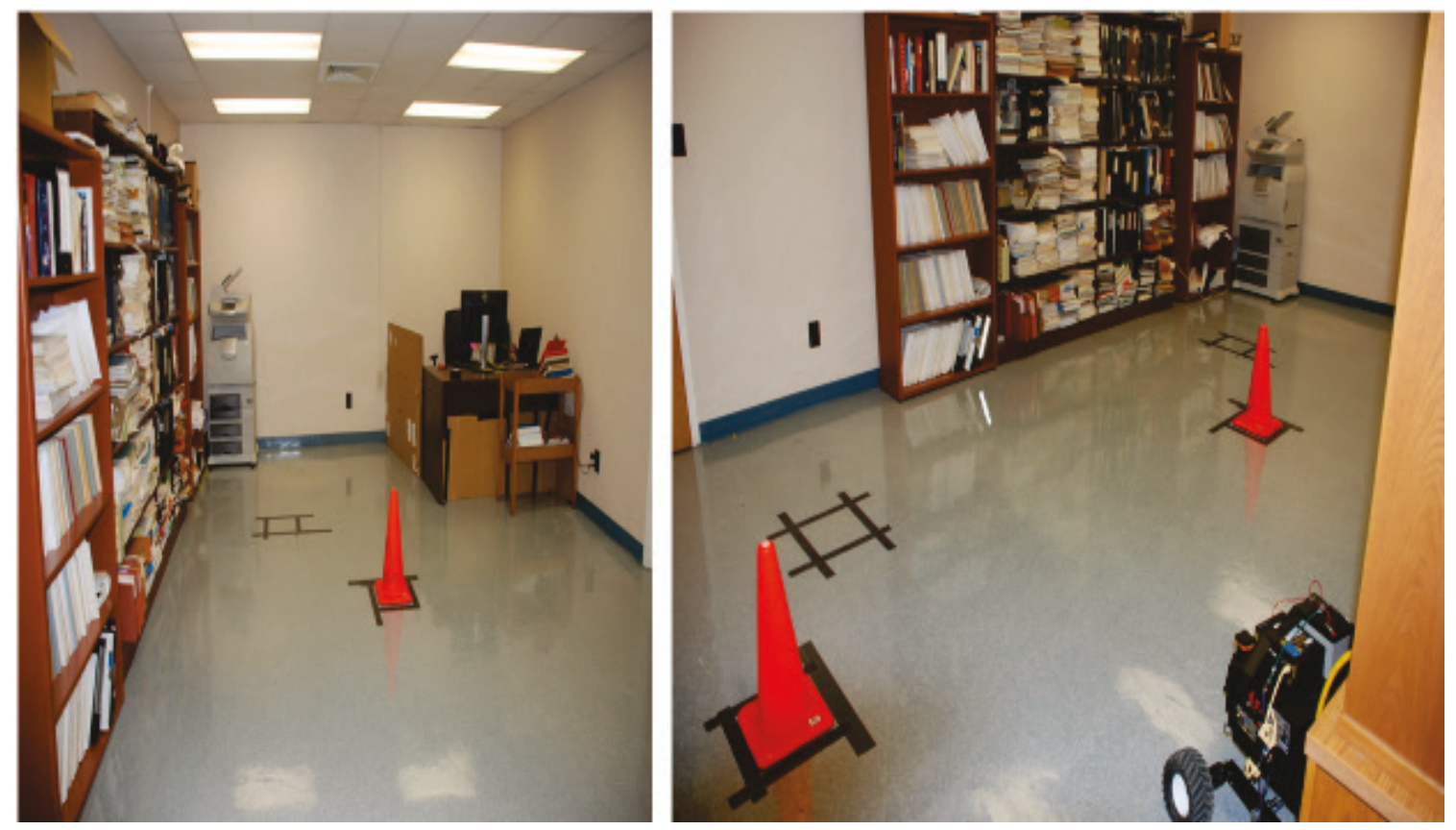

Next, the generated maps were imported into GIMP 2 and scaled to resolution size. For this test, the resolutions tested were $200 \times 200$, $300 \times 300$, and $500 \times 500$. Resolution $100 \times 100$ produced a poor quality map and was not on the canvas; thus testing for $100 \times 100$ resolution was eliminated. A map of resolution $1000 \times 1000$ created an excellent map, but this resolution was too memory intensive for the $\mathrm{A}^{*}$ package and would core dump. The $\mathrm{x}$ and $\mathrm{y}$ positions for each of the points were marked as in 
the previous steps. The two points are shown in the figures below and are denoted for each of the resolutions. Figure 39 shows Point A and B along with their $\mathrm{x}$ and $\mathrm{y}$ coordinates for resolution $300 \times 300$.

Figure 39. Gimp $300 \times 300$.

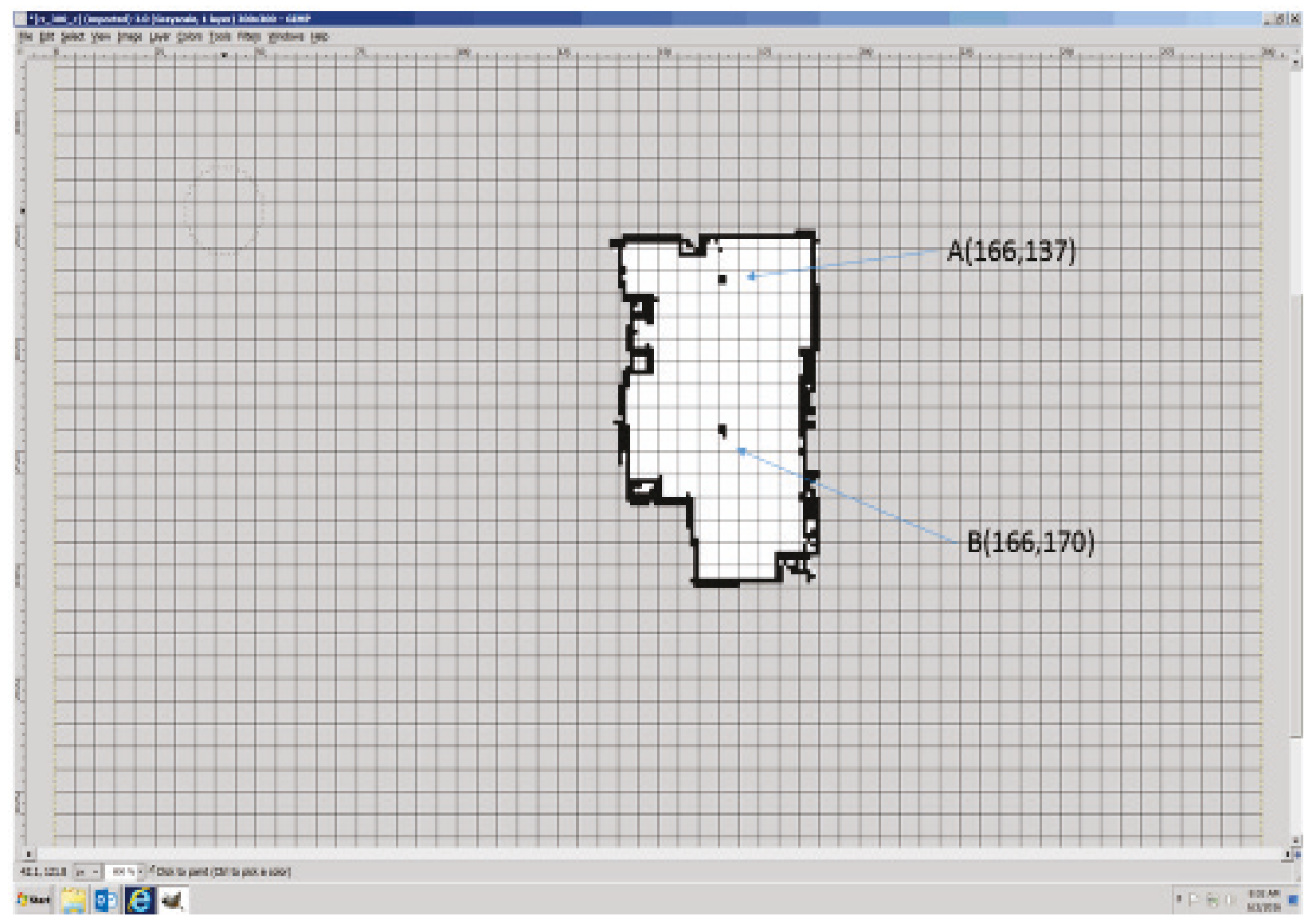

When running the $\mathrm{A}^{*}$ algorithm, the start point $\mathrm{A}$ was provided by AMCL, and the destination Point B was provided from the Gimp software. The room was larger than in Test Site One in Tables 2 to 14. The distance from where the robot ended and the position of Point B in the physical map is shown in the Distance column, and the time to travel the path is in the time columns. In the $200 \times 200$ resolution map, Table 12 , the error was from 48 to 70 in., with an average of $61 \mathrm{in.} \mathrm{for} \mathrm{the} \mathrm{five} \mathrm{runs.} \mathrm{In} \mathrm{the}$ $300 \times 300$ resolution map, Table 13 , the error was from 35 to 80 in., with an average of $55.4 \mathrm{in}$. for the five runs. In the $500 \times 500$ resolution map, Table 14, the error was from 56 to 108 in., with an average of 69.6 in. for the five runs. Overall, for Test Site Three, the $300 \times 300$ resolution performed best. 
Table 12. Results for $200 \times 200$ on Test Site Three.

\begin{tabular}{|l|l|l|l|l|l|}
\hline & \multicolumn{2}{|c|}{ Generated Map } & AMCL & & \\
\hline & A & B & A & Time (sec.) & Distance (in.) \\
\hline Run 1 & $(116,95)$ & $(107,128)$ & $(110,110)$ & 26.34 & 60 \\
\hline Run 2 & $(116,95)$ & $(107,128)$ & $(110,110)$ & 25.89 & 63 \\
\hline Run 3 & $(116,95)$ & $(107,128)$ & $(110,110)$ & 26.05 & 64 \\
\hline Run 4 & $(116,95)$ & $(107,128)$ & $(120,110)$ & 13.79 & 48 \\
\hline Run 5 & $(116,95)$ & $(107,128)$ & $(110,110)$ & 27.75 & 70 \\
\hline
\end{tabular}

Table 13. Results for $300 \times 300$ Test Site Three.

\begin{tabular}{|l|l|l|l|l|l|}
\hline & \multicolumn{2}{|c|}{ Generated Map } & AMCL & \\
\hline & A & B & A & Time (sec.) & Distance (in.) \\
\hline Run 1 & $(166,137)$ & $(166,170)$ & $(160,150)$ & 29.62 & 36 \\
\hline Run 2 & $(166,137)$ & $(166,170)$ & $(160,150)$ & 38.93 & 60 \\
\hline Run 3 & $(166,137)$ & $(166,170)$ & $(160,150)$ & 29.08 & 35 \\
\hline Run 4 & $(166,137)$ & $(166,170)$ & $(170,150)$ & 30.83 & 66 \\
\hline Run 5 & $(166,137)$ & $(166,170)$ & $(170,170)$ & 32.17 & 80 \\
\hline
\end{tabular}

Table 14. Results for $500 \times 500$ Test Site Three.

\begin{tabular}{|l|l|l|l|l|l|}
\hline & \multicolumn{2}{|c|}{ Generated Map } & AMCL & \\
\hline & A & B & A & Time (sec.) & Distance (in.) \\
\hline Run 1 & $(269,248)$ & $(248,276)$ & $(250,250)$ & 41.91 & 108 \\
\hline Run 2 & $(269,248)$ & $(248,276)$ & $(260,260)$ & 26.31 & 62 \\
\hline Run 3 & $(269,248)$ & $(248,276)$ & $(260,260)$ & 23.12 & 56 \\
\hline Run 4 & $(269,248)$ & $(248,276)$ & $(260,250)$ & 40.56 & 62 \\
\hline Run 5 & $(269,248)$ & $(248,276)$ & $(260,250)$ & 36.51 & 60 \\
\hline
\end{tabular}

\subsection{Conclusion}

This research shows that the resolution of the map has an effect on the robot's localization and path finding ability. The Explorer performed best at $300 \times 300$ resolution in all cases.

The research in Chapter 6 provides the ERDC with an increased level of autonomy from zero or tethered to autonomous level three, when searching unknown environments. When this research began, the ERDC 
was using a tethered robot to explore unknown areas. We have tested and selected the SLAM algorithm via simulation, performed physical testing on a mobile robot and concluded that HectorSLAM is an excellent choice for mapping an unknown area. Also, a ROS package AMCL, was implemented, which allows the robot to localize itself to the SLAM generated map. Once localized to the map, the mobile robot was able to traverse a path generated with the $\mathrm{A}^{*}$ algorithm. 


\section{Conclusions and Future Work}

\subsection{Conclusions}

The main goals of this dissertation were to enhance a commercial off the shelf (COTS) robotic platform with the ability to generate a map of an unknown area, to localize the robot to the generated map, and to increase the level of autonomy from tethered to a level of five or more. The UGV was tested with three SLAM algorithms, CORESLAM, Hector SLAM, and Gmapping, in a simulated environment and a physical environment. Hector SLAM proved to be the superior SLAM algorithm, outperforming both Gmapping and CORESLAM in both simulation testing and physical testing. It is my belief that Hector SLAM generated the best map because it requires only a laser scan, unlike Gmapping and CORESLAM.

This research had a series of limitations: (1) ROS has a steep learning curve and there are unlimited packages for use, (2) ROS operates in a Linux environment, (3) lack of a dedicated test area that caused multiple moves and setups as time allowed, and (4) the UGV and laptop's WIFI signals proved unacceptable for the transmission of data. This WIFI issue was overcome by adding a radio to both the laptop and UVG and setting up a network between the two. Large amounts of data were then transmitted between the two.

\subsection{Future work}

Further enhancements to the UGV include adding other plug-and-play devices such as Microsoft Kinect Sensor or the 360-deg 3-D sensor by DFROBOT. Each of these sensors will produce a 3-D map of the navigated area that can be overlaid onto the 2-D map. The Microsoft Kinect sensor costs about $\$ 100$ and is easily installed. The Kinect consists of several sensors including a RBG sensor, a 3-D depth sensor, multi-array microphone, and an accelerometer (https://developer.microsoft.com/enus/windows/kinect/hardware). The 360-deg 3-D sensor by DFROBOT is also inexpensive and should be easily programmed.

Increasing the level of autonomy from around three, to between six and ten is needed. Testing in a tunnel environment would show more of the strengths and weaknesses of this UGV. 


\section{References}

Angeli, A., S. Doncieux, J. A. Meyer, and D. Filliat. 2008. Incremental vision-based topological SLAM. Intelligent Robots and Systems, IEEE/RSJ International Conference. Nice, France.

Banks, J., and J. S. Carson. 1984. Discrete event system simulation. Upper Saddle River, NJ: Prentice-Hall.

Dellaert, F., D. Fox, W. Burgard, and S. Thrun. 1999. Monte Carlo localization for mobile robots. In Proceedings, IEEE International Conference on Robotics and Automation. 10-15 May, Detroit, MI.

Doray, E., A. Clymer, J. McKenna, D. Horner, M. McKenna, T. Anderson, et al. 2009. Unmanned tunnel exploitation. Technologies for Homeland Security, IEEE Conference. Waltham, MA.

Edwards, D., T. Bean, D. Odell, and M. Anderson. 2004. A leader-follower algorithm for multiple AUV formations: IEEE. In 2004 IEEE/OES Autonomous Underwater Vehicles. 17-18 June, Sebasco, ME.

Fox, D., W. Burgard, and S. Thrun. 1999. Markov localization for mobile robots in dynamic environments. Journal of Artificial Intelligence Research 11(3):391-427.

Gage, D. W. 1995. UGV history 101: A brief history of unmanned ground vehicle development efforts. Unmanned Systems Magazine 13(3).

Kinney, P., M. Dooner, J. Nagel, and P. Trepagnier. 2006. Kat-5: Systems based on a successful paradigm for the development of autonomous ground vehicles. In 2006 IEEE/ION Position, Location, And Navigation Symposium. 25-27 April, Coronado, CA.

Kohlbrecher, S. 2011. Hector_mapping. http://wiki.ros.org/ hector_mapping.

Kramer, J. A. 2010. Accurate localization given uncertain sensors. MS thesis, University of South Florida.

Morgan Quigley, B. G., K. Conleyy, J. Fausty, T. Footey, J. Leibsz, E. B., R. Wheelery, and A. Ng. 2009. ROS: an open-source Robot Operating System. ICRA Workshop on Open Source Software. Kobe, Japan.

Murphy, R. R. 2004. Rescue robotics for homeland security. Communications of the ACM 47(3):66-68.

Reynolds, J. 2005. An exploration of mapping algorithms for autonomous robotic mapping. Colorado State University, Department of Mechanical Engineering.

Riisgaard, S., and M. R. Blas. 2004. SLAM for dummies. A tutorial approach to simultaneous localization and mapping. Cambridge, MA: MIT OCW. 
Rozman, J. 2009. Grid-based map making using particle filters. International Journal of Autonomic Computing 1(2):211-221.

Russell, S., and P. Norvig. 1995. A modern approach. In Artificial Intelligence. Englewood Cliffs, CA: Prentice-Hall.

Santos, J. 2008. Gmapping. http://wiki.ros.org/gmapping.

Singer, P. W. 2008. Robots at war: The new battlefield. Wilson Quarterly 30-48.

Song, I. 2002. Probabilistic localization methods for a mobile robot navigation. University of Waterloo.

Stanculescu, A., and M. Sojka. 2008. Evaluation of the Monte Carlo localization algorithm. Prague: Czech Technical University.

Staranowicz, A., and G. Mariottini. 2011. Robotic simulation guide. ASTRA Robotics Lab, Department of Computer Science and Engineering, University of Texas. http://ranger.uta.edu.

Thrun, S. 2002. Robotic mapping: A survey. In Exploring artificial intelligence in the new millennium, $1-35$.

Thrun, S. 2006. The graph SLAM algorithm with applications to large-scale mapping of urban structures. The International Journal of Robotics Research 25(5-6):403429 .

Thrun, S., D. Fox, W. Burgard, and F. Dellaert. 2001. Robust Monte Carlo localization for mobile robots. Artificial Intelligence 128(1-2):99-141.

University of Tennessee Knoxville. 2007. Player/stage: Getting started guide. https://mabe.utk.edu/.

Voth, D. 2004. A new generation of military robots. Intelligent Systems, IEEE 19(4):2-3.

Welch, G., and G. Bishop. 1995. An introduction to the Kalman filter. University of North Carolina at Chapel Hill. 
Appendix A: Mobile Robot Cost 


\section{Groware}

Coroware

1410 Market Street

Kirkland, WA 98033

Phone: (800) 641-2676, option 1

BILL TO:

U.S. Army Corps of Engineers

Attn: Doris Turnage

\section{DATE: $\quad$ February 15, 2011 \\ ESTIMATE: $\quad$ EXPLORER - USAE \\ TERMS: Net 30}

SHIP TO:

U.S. Army Corps of Engineers

Attn: Doris Turnage

\begin{tabular}{|c|c|c|c|c|c|}
\hline \multirow{2}{*}{\begin{tabular}{|ll} 
& DESCRIPTION \\
Explorer Mobile Robot &
\end{tabular}} & \multirow{2}{*}{$\frac{\text { Quantity }}{1}$} & \multicolumn{2}{|c|}{ Amount } & \multicolumn{2}{|c|}{ Total } \\
\hline & & $\$$ & $8,499.00$ & $\$$ & $8,499.00$ \\
\hline Power Options : Field "Hot Swap", Lithium Ferrous Phosphate (LiFePO) & 1 & $\$$ & - & $\$$ & - \\
\hline Sensors: GPS Receiver, 2 MegaPixel Color WebCam & 1 & $\$$ & - & $\$$ & - \\
\hline Mainboard Options: CUDA-capable & 1 & $\$$ & 600.00 & $\$$ & 600.00 \\
\hline Operating System: Ubuntu Linux & 1 & $\$$ & 100.00 & $\$$ & 100.00 \\
\hline Robotics Software Platform : Robot Operating System (ROS) & 1 & $\$$ & 200.00 & $\$$ & 200.00 \\
\hline Storage media: 60 Gigabyte solid-state drive & 1 & $\$$ & 300.00 & $\$$ & 300.00 \\
\hline Accessories: Pan/tilt camera & 1 & $\$$ & 750.00 & $\$$ & 750.00 \\
\hline Laser Range Finder: Outdoor LRF UTM-30LX & 1 & $\$$ & $6,999.00$ & $\$$ & $6,999.00$ \\
\hline Battery Pack (Lithium Ferrous Phosphate (LiFePO); estimated 6-10 hours operation) & 1 & $\$$ & 700.00 & $\$$ & 700.00 \\
\hline Intelligent Battery Charger and Conditioner & 1 & $\$$ & 200.00 & $\$$ & 200.00 \\
\hline Mainboard Options: CUDA-capable & 1 & $\$$ & 600.00 & $\$$ & 600.00 \\
\hline Subtotal & & & & $\$$ & $18,948.00$ \\
\hline Shipping (Within USA) & 1 & $\$$ & 350.00 & $\$$ & 350.00 \\
\hline \multirow[t]{2}{*}{ WRE TRANSFER INFORMATION: } & & & \multirow{2}{*}{$\begin{array}{l}\text { SUBTOTAL } \\
\text { TAX RATE } \\
\text { SALES TAX }\end{array}$} & $\$$ & $\begin{array}{r}19,298.00 \\
0.00 \%\end{array}$ \\
\hline & & & & $\$$ & - \\
\hline \multirow{2}{*}{$\begin{array}{l}\text { Wells Fargo Bank } \\
\text { Account Owner: CoroWare Technologies Inc } \\
\text { Account Number: } 1608861744\end{array}$} & & & \multirow{2}{*}{$\begin{array}{l}\text { OTHER } \\
\text { TOTAL }\end{array}$} & & \\
\hline & & & & $\$$ & $19,298.00$ \\
\hline
\end{tabular}

Account Number: 1608861744

SWIFT Code: WFBIUS6S

This quote expires 90 days after the date of issue. 


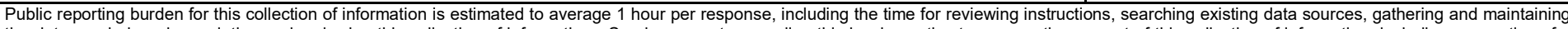

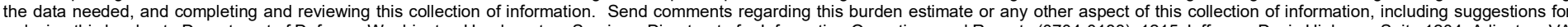

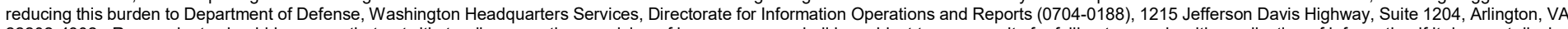

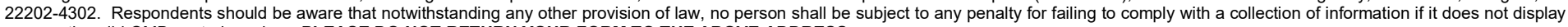
a currently valid OMB control number. PLEASE DO NOT RETURN YOUR FORM TO THE ABOVE ADDRESS.

\begin{tabular}{l|l|l} 
1. REPORT DATE (DD-MM-YYYY) & 2. REPORT TYPE & 3. DATES COVERED (FrOm - To)
\end{tabular} February 2019 Final

\section{TITLE AND SUBTITLE}

Localization and Mapping of Unknown Locations with Unmanned Ground Vehicles

5a. CONTRACT NUMBER

5b. GRANT NUMBER

5c. PROGRAM ELEMENT NUMBER

\section{AUTHOR(S)}

Doris M. Turnage 5d. PROJECT NUMBER

5e. TASK NUMBER

5f. WORK UNIT NUMBER

8. PERFORMING ORGANIZATION REPORT NUMBER

ERDC/GSL TR-19-3

U.S. Army Engineer Research and Development Center

3909 Halls Ferry Road

Vicksburg, MS 39180-6199

\section{SPONSORING / MONITORING AGENCY NAME(S) AND ADDRESS(ES)}

U.S. Army Engineer Research and Development Center

3909 Halls Ferry Road

Vicksburg, MS 39180-6199

11. SPONSOR/MONITOR'S REPORT NUMBER(S)

\section{DISTRIBUTION / AVAILABILITY STATEMENT}

Approved for public release; distribution is unlimited.

\section{SUPPLEMENTARY NOTES}

\section{ABSTRACT}

The main goals of this research are to enhance a commercial off the shelf (COTS) software platform to support unmanned ground vehicles (UGVs) exploring the complex environment of tunnels, to test the platform within a simulation environment, and to validate the architecture through field-testing. Developing this platform enhances the U.S. Army Engineering Research and Development Center's (ERDC's) current capabilities and creates a safe and efficient autonomous vehicle to perform the following functions within tunnels: (1) localizing (e.g., position tracking) and mapping of its environment, (2) traversing varied terrains, (3) sensing the environment for objects of interest, and (4) increasing the level of autonomy of UGVs available at the ERDC. The simulation experiments were performed in the STAGE Simulator, a physics-based, multi-scale numerical test bed developed by Robotic Operating System (ROS). Physical testing was conducted in Vicksburg, MS, using a Coroware Explorer. Both the simulation and physical testing evaluated three simultaneous localization and mapping (SLAM) algorithms, i.e., Hector SLAM, Gmapping, and CORESLAM to determine the superior algorithm. The superior algorithm was then used to localize the robot to the environment and autonomously travel from a start location to a destination location. Completion of this research has increased the ERDC's level of autonomy for UGVs from tether to tele-operated to autonomous.

\begin{tabular}{|c|c|c|}
\hline \multicolumn{2}{|c|}{ 15. SUBJECT TERMS } & Tunnels \\
\hline \multicolumn{2}{|c|}{ Autonomous vehicles } & Underground areas \\
\hline \multicolumn{2}{|c|}{ Vehicles, military } & Navigation \\
\hline \multicolumn{3}{|c|}{ 16. SECURITY CLASSIFICATION OF: } \\
\hline a. REPORT & b. ABSTRACT & c. THIS PAGE \\
\hline Unclassified & Unclassified & Unclassified \\
\hline
\end{tabular}

Cartography - Software

Maps

17. LIMITATION
OF ABSTRACT

\section{NUMBER} OF PAGES

75 19a. NAME OF RESPONSIBLE PERSON

19b. TELEPHONE NUMBER (include area code) 